THE NUMERICAL SIMULATION OF HYSTERETIC:

FLOW IN POROUS MEDIA

By

ATTILA. I. EVRENOS

Bachelor of Science University of Missouri at Rolla

Rolla, Missouri 1961

Master of Science

Ok.lahoma State University

Stillwater, Oklahoma 1967

Submitted to the Faculty of the

Graduate College of the

OkIahoma State University

in partial fulfillment of

the requirements for

the Degree of

DOCTOR OF PHILOSOPHY

May, 1970 


$$
\begin{aligned}
& \text { Thesis } \\
& 19700 \\
& \text { E93m } \\
& \text { Cop.2 }
\end{aligned}
$$




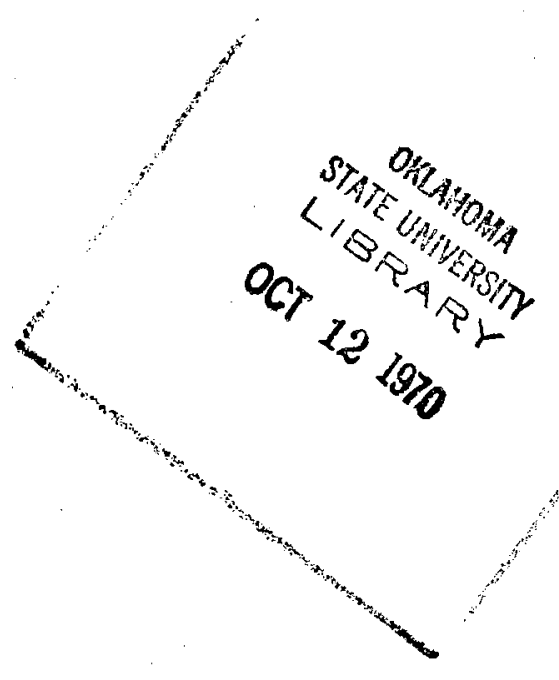

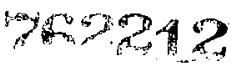

\footnotetext{
Copyright by Attila I. Evrenos 1970
} 
THE NUMERICAL SIMULATION OF HYSTERETIC

FLOW IN POROUS MEDIA

Thesis Approved:
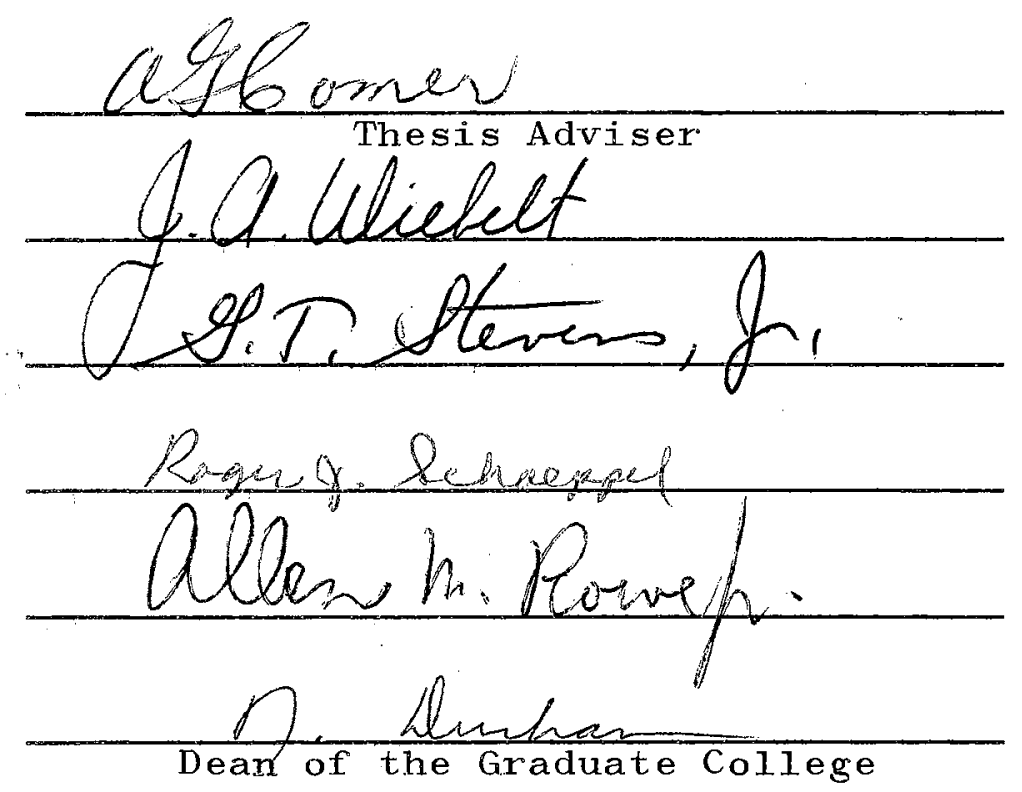


\section{PREFACE}

This dissertation is concerned with the development of a numerical simulator for the three-dimensional flow of two immiscible, compressible fluid phases in porous media, including the effects of gravity, capillarity, heterogeneity, and hysteresis in relative permeability and capillarity. The primary objective of the research project was the development of an evaluation-prediction tool for gas recovery from gas fields under active water drive, cyclic gas movement in aquifer gas storage and, in particular, to determine the quantity of residual gas remaining in an aquiferreservoir complex at abandonment. The secondary objective was the investigation of the qualitative and quantitative effects of rigorous representation of hysteretic relative permeability and capillarity, on the numerically computed saturation and pressure distributions.

A set of two, second-order, non-linear, parabolic partial differential equations governing two-phase, hysteretic flow were derived. An analogous system of difference equations were developed for numerical solution by an alternating direction implicit iterative method. Semiempirical relationships spanning the domain of relative permeability and capillarity data were derived from laboratory data and statistical-capillaric models of the porous media. 
Parametric sensitivity studies of the effects of relative permeability and capillarity on the numerical calculation of fluid saturation-pressure distributions were made by simulation of hypothetical systems representing typical prototype reservoirs.

I would like to take this opportunity to express my appreciation for the assistance and guidance given me by the following members of my committee: Professor A. G. Comer, Thesis Adviser, who was always available for counsel, encouragement, and professional cooperation; Dr. J. A. Wiebelt, Committee Chairman; Doctors A. M. Rowe, R. J. Schoeppe1, and G. T. Stevens, Committee Members, for their interest and assistance.

I wish to thank the Northern Natural Gas Company of Omaha; Nebraska, for full sponsorship of this research project. I appreciate the interest and the assistance of Messrs. F. D. Stockman, E. V. Martinson, L. N. Reed, and A. Baca and his staff, of Northern.

My appreciation also goes to Mr. R. D. Grimm, Consultant; to Dr. J. R. Dempsey and Dr. K. H. Coats of the University of Texas; and to Mr. J. H. Henderson of International Computing Applications, Inc., for their personal interest and suggestions.

In addition, I offer my thanks to Mrs. Sherry Kreger, Mrs. Melba Greene, Miss Sandy Alm, Mrs. Marian Cole and Mrs. Patricia Vanoutry for their typing excellence and advice on the various drafts of this thesis, and to Miss Velda Davis 
for the final typing of this manuscript, and to Mr. M. B. Lehman for his superior drafting.

Finally, I would like to express my deep appreciation to my wife, Lynne, my son, Pars, and my parents, whose understanding, encouragement, and sacrifice were invaluable in the preparation of this dissertation. 
TABLE OF CONTENTS

Chapter

Page

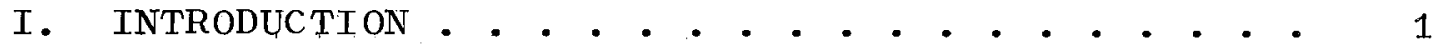

II. LITERATURE SURVEY • . . . . . . . . . . . . . 6

III. STATEMENT OF THE PROBLEM • . • • . . . . . . 12

IV . GENERATION OF HYSTERETIC CAPILLARY

PRESSURE AND RELATIVE PERMEABILITY

CURVES . . . . . . . . . . . . . . . 16

Hysteretic PC Model. . . . . . . . 16

Hysteretic RP Model . . . . . . . . . 24

Semi-Empirical Relations for

Hysteretic RP and PC . . . . . . . 29

V. DESCRIPTION OF THE NUMERICAL MODEL . • • • • . 36

Partial Integration of Equations

of Two-Phase Flow . . . . . . . . . 39

VI. APPLICATION OF THE NUMERICAL SIMULATOR:

SENSITIVITY STUDIES OF RP AND PC IN

RESERVOIR MODELING . • . . . . . . . . . . 4 42

VIII. SUMMARY, CONCLUSIONS, AND RECOMMENDATIONS • • • 51

BIBLIOGRAPHY • • • . • . • • • • • • . . . 58

APPENDIX A - EQUATIONS OF INTERMEDIATE DRAINAGE -

IMBIBITION RP AND PC . . . . . • • • . . 65

APPENDIX B - FLOW EQUATIONS GOVERNING THE THREEDIMENS IONAL, TWO-PHASE, C OMPRESS IBLE, IMMISCIBLE, HYSTERETIC FLOW IN

POROUS MEDIA • • . . . . . . • • • . 69

APPENDTX C - HYSTRACK ALGORITHM • • • • • • • • 123

APPENDIX D - PARTIAL INTEGRATION OF EQUATIONS OF

TWO-PHASE FLOW--VERTICAL EQUILIBRIUM

( VE ) • . . . . . . . . . . . . . 128 
APPENDIX E - PARAMETRIC SENSITIVITY STUDIES OF RP AND PC IN RESERVOIR

MODELING . . . . . . . . . . . . 132

APPENDIX F - SYMBOL LIST FOR THE TEXT - • • • • • • 167 


\section{LIST OF TABLES}

Table

Page

I. Saturation $\left(S_{n}\right)$ Maps for the Example Simulation Run A1 . . . . . . . ... . . 43

II. Pressure Profiles for Example Simulation Run A1 (psi) . .............. . . . 44

III. Saturation ( $\left.S_{k}\right)$ Map for Run B1 at the Simulation Time Level of 763 Days . . . . . . 135

IV. Saturation $\left(S_{w}\right)$ Profiles for Runs $B 5$ and B6 at the Simulation Time Level of 763 Days . . . . . . . . . . . . . 145

V. Saturation $\left(S_{w}\right)$ Profiles for Runs $C_{1}, C_{2}$, C3 at Simulation Time of 313 Days and Run D1 at 823 Days . . . . . . . . . . 153

VI. Saturation-Pressure Data From Runs F1-F5 at Simulation Time Level of 50 Days . . . . . 164 
LIST OF FTGURES

Figure

Page

1. Typical Relationships Between Capillary

Pressure and Saturations of Wetting

and Non-Wetting Phases . . . . . . . . . . 19

2. Cyclic Capillary Pressure, Core A1-S1 . . . . . 20

3. Cyclic Capillary Pressure, Core H1-F3 . • . . . . 21

4. Growth in Capillary Pressure Envelope . . . . . 23

5. Relative Permeability Curves for (a)

Consolidated Sands (b) Unconsolidated

Glass Spheres (Reproduced From Naar-

Henderson (64) Original Article) . . . . . . . 25

6. Relative Permeability, Core A1-S1 . . . . . . . 27

7. Relative Permeability, Core H1-F 3 . . . . . . . 28

8. Bounding Drainage Relative Permeability

Curves Generated by Equations (6) and (7) . . . 32

9. Various Paths for Bounding Imbibition

Capillarity Curves Generated by

Equation (13) . . . . . . . . . . . . 35

10. Two-Dimensional Grid Network Used in

Simulation Run Groups A, B, C, D . . . . . . . 45

11. Injection-Production Schedule for Each Well

in Simulation Run A1 . . . . . . . . . . 46

12. Flow Chart for Hystrack Algorithm . . . . . . 125

13. Loci of Non-Wetting Phase Relative Permeability

Calculated by Hystrack Algorithm . . . . . . 127

14. Linear and Curvilinear Capillary Pressure

Curves Used in Injection and Production

Simulation Studies... . . . . . . . . . 134 
15. Water Saturation Versus Simulation Time P1ots for Runs B1, B2, and B3 [Blocks $(5,1)$ and $(5,5)]$. . . . . . . . . .

16. Water Saturation Versus Simulation Time Plots for Runs B1, B2, and B3. [B1ocks

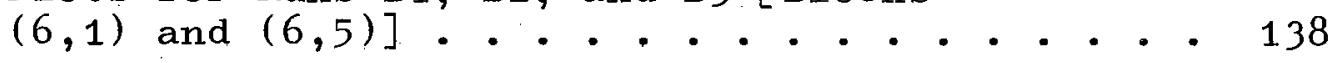

17. Water Saturation Versus Simulation Time Plots for Run B4 [Blocks $(5,1)$, $(5,5)$, $(6,1)$, and $(6,5)]$. . . . . . . . . 140

18. Dynamic Block Pressure Versus Simulation Time Plots for Runs B1 and B4 . . . . . . . 142

19. Water Cut Per Well in Simulation Runs C1,

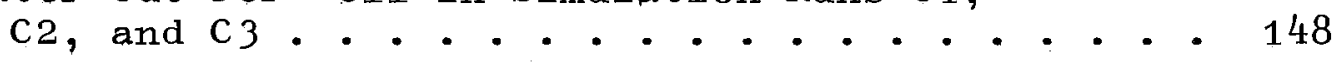

20. Production Schedules of We11s in Simulation Runs C1 and D1 . . . . . . . . . . . 152

21. Sensitivity of Equilibrium Capillary Pressure to Depth of Partial Integration in DipNormal Direction, $\mathrm{H}_{z}$ - . . . . . . . . . 155

22. Sensitivity of Equilibrium Relative Permeability to Depth of Partial Integration in Dip-Normal Direction, $\mathrm{H}_{z}$. . . . . . .

23. Two-Dimensional Grid Network Used in Simulation Run E1 . . . . . . . . . . 161

24. One-Dimensional Radial Grid Network Used in Simulation Run F3................

25. Saturation Distribution About the Injection Well at Various Levels of Run F3 Simulation Time... . . . . . . . . . . . . . 
CHAPTER I

\section{INTRODUCTION}

During the last decade there has been an increased realization of the need for refined, quantitative answers concerning the behavior of hydrocarbon reservoirs and their management. The exploitation of these reservoirs continues to be accelerated to meet the ever growing demand for energy resources. As the exploitation of the known reservoirs intensifies and the discovery of the significant new reservoirs becomes more difficult; the technologists and their management become more interested in the response of the known reservoirs to advanced recovery techniques, whereas the initial concern has been the delineation and exploration of these reservoirs. Due to intense competition, both in exploration and exploitation, and gradual exhaustion of hydrocarbons through production, the principal problem confronting the reservoir technologists today is one of proper reservoir management to achieve optimum recovery. Before these energy resources can be managed, they must be quantitatively appraised so that the proposed exploitation techniques can be evaluated.

Emphasis is now placed on the quantitative description of the geologic, and dynamic fluid properties affecting the 
hydrocarbon energy source yielding capacity of wells and reservoirs. Research is being directed toward developing and improving analytical and numerical methods of simulating the response of wells and reservoirs to various exploitation techniques.

The primary objective of the research which is the basis of this dissertation has been the development of a numerical simulator for the three-dimensional flow of two immiscible, compressible fluid phases in porous media, including the effects of gravity, capillarity, and hysteresis in relative permeability and capillarity. The second objective was the investigation of the qualitative and quantitative effects of rigorous representation of hysteresis in capillarity and relative permeability on the model to determine whether and when such rigor is worthwhile.

One of the most important problems in the design of gas storage fields is that of determining or estimating the individual and cumulative deliverabilities and injectabilities of wells as a function of time, gas-in-place, and injectionwithdrawal patterns. In order to do this, reservoir pressure and saturation distribution must be determined or predicted as a function of time, which requires the use of a suitable simulator. The prediction of fluid movement by a simulator is also important in estimating the most advantageous positions of new wells, exploitation rates to which they can be subjected, and the position of the gas-water transition zone during periods of high and low reservoir 
pressure and gas-in-place to avoid gas movement beyond the "spill point", and watering (or coning) of wells. It must be pointed out that gas reservoirs naturally developed in aquifers over a period of geologic time offer just as much challenge to technologic and economic ingenuity of man as the gas storage reservoirs, except that the initial drainage process has been accomplished by nature and there is usually no cycling of gas. This dissertation will be concerned with the simulation of reservoirs containing two immiscible, compressible fluids with particular emphasis on technologically more general, cyclic storage reservoirs.

Such a simulator is also applicable to oil-water systems in the vicinity and above the bubble-point, and to gasoil systems subject to sufficiently small pressure gradients.

Analytical and pseudo-analytical methods have heretofore been applied to relatively uniform reservoir-aquifer systems with simple geometry only. More complex systems defy the capabilities of these techniques, or, in some cases, the computational effort becomes astronomical. These techniques are we1l documented in the Society of Petroleum Engineers (SPE) 1iterature. Earlier referenced work of the author provides the most recent mathematical description and field applications (1, 2, 3).

Numerical simulators have emerged in response to the need for detailed studies, and the failure of convenient classical methods, such as the separation of variables 
techniques or Green's functions, in solving the differential analogs of the multi-dimensional, multi-phase flow systems. Today, generalized simulators are available to most reservoir technologists and development work continues with ever increasing tempo: However, the realism and the versatility of analytical or numerical simulators depend largely upon the availability of abundant and accurate data. Frequently, basic data are not sufficient to warrant a rigorous description of a complex system by numerical analogs, and in such cases simpler, faster and cheaper analytical methods may be just as useful.

The advantage of a numerical simulator is, at least, twofold. Firstly, it provides answers where analytical techniques fail. Secondly, it provides a complete pressure and saturation information at each point in time and space which is beyond the capabilities of present analytical simulators.

Various methods $(4-7)$ of simulating the flow of one to three fluids within porous media and in one to two spatial dimensions have been reported. To the author's knowledge, the only numerical simulator for two-phase, immiscible, compressible, three-dimensional flow in porous media, is the one reported by coates et al. (8). However, this simulator does not incorporate the effects of capillarity and relative permeability hysteresis. Recent investigations show that relative permeabilities and capillary pressures are functions of phase saturations (10-14). Tests 
performed by Comer (15) demonstrated that relative permeability and capillary pressure depend upon the number of drainage-imbibition cycles the porous media are subjected to, and therefore time (saturation history) also becomes a factor. The importance of hysteresis in dynamic and static capillary equilibrium in porous materials was stressed in recent excellent papers by Melrose (16), and by Morrow and Harris (17)。

A number of gas-water reservoir simulation studies have been performed in this research to test the accuracy and validity of the simulator, as well as its sensitivity to the methods of representing relative permeability and capillary pressure data. Specific initial drainage, initial imbibition, and cyclic gas injection-withdrawal simulations are presented in this dissertation together with their appraisals. 


\section{CHAPTER II}

\section{LITERATURE SURVEY}

In 1856, French hydrologist Henry Darcy (18) demonstrated the existence of a parameter characterizing the fluid conductivity of porous materials. The equation which defined "permeability" as fluid conductivity in porous media in terms of measurable quantities became known as Darcy's Law. The unit of permeability was named Darcy in honor of its originator. Later, Hubbard generalized the Darcy Law and showed how it could be derived from Navier-Stokes equations (19). In general form, Darcy's Law is written as:

$$
\bar{v}=-\frac{\varphi}{B \mu} \nabla \bar{\Phi}=-\frac{\varphi}{B \mu} \nabla p+\hat{I}_{3} \rho g
$$

where, $\varphi / B$ is the permeability and it is generally represented by the letter $k$. $I_{3}$ is a unit vector taken positive upward. Symbol list for the text is given in Appendix F. Until early 1930's integrals of this law were applied to hydrocarbon reservoirs assuming single phase flow. Experimental work in the early $1930^{\prime} \mathrm{s}$ showed that the permeability to each phase was reduced when more than one fluid phase was present in porous materials $(20,21)$. In a celebrated publication Muskat and Meres (22) postulated the concept of relative permeability. The corresponding form of 
Darcy's Law can be written as:

$$
\bar{v}_{\hat{p}}=-k \frac{k_{p_{p}}}{\mu_{p}} \nabla \bar{\Phi}_{p}
$$

where $f$ denotes a particular fluid phase.

Early drainage experiments showed that the relative permeabilities depend only on the saturation of fluid phases within the samples of porous media tested (23, 24). The product $\left(\mathrm{kk}_{\mathrm{r}_{\mathrm{f}}}\right)$ became known as the effective permeability to phase f. A multitude of papers have been published since the introduction of this concept (13, 14, 24, 25, 26). Much work has been done in this area by comer et al. $(10-15)$

Later, it was realized that distinct relative permeabilities versus phase saturation curves were obtained during drainage-imbibition tests, indicating the importance of the direction of phase saturation changes (10, 11, 12, 13, 14, 24, 25). Comer's (15) recent work showed that hysteresis itself is influenced by the number of drainageimbibition cycles imposed on porous media.

The concept of capillary pressure in multi-phase fluid flow in porous media was introduced by Leverett (27). The capillary pressure is the differenoe in pressure across a fluid-fluid interface, and it is caused by the interfacial tension. Although the capillary pressure can be calculated from LaPlace's well-known equation,

$$
P_{0}=\sigma\left[\left(1 / r_{I}\right)+\left(1 / r_{2}\right)\right]
$$


or from other expressions involving interfacial tension and contact angle (measure of wettability), such equations are not used in macroscopic treatment of porous media. Most of the elements in these equations are very hard to measure, if not presently impossible; furthermore, the very complex and tortuous nature of porous media in microscopic view, and variations in wettability from point-to-point and with time makes such equations impractical. As in the case of relative permeability, capillary pressure has successfully been correlated with phase saturation in the laboratory. Hysteresis phenomenon has been observed in capillary pressure versus saturation plots, including the effects of cycling (15). Three excellent papers by Pickell et al. (28) Morrow and Harris (17), and Melrose (16) on the capillary pressure hysteresis are referenced.

Buckley and Leverett (29) derived the "fractional flow equations" for the flow of immiscible fluid phases in onedimensional media, in 1942. Since their introduction, these equations, together with the concepts of relative permeability and capillary pressure, have been used in one form or another in numerous analytical and numerical simulators.

Until the advent of digital computers which enhanced the power and the practicality of numerical methods, simulators were limited to solutions of basic differential equations governing fluid flow in porous media for 1 inear and radial systems.

It appears that the first simulation work employing 
numerical methods and computing machines was done by Terwillinger et al. (30) in 1951. These investigators applied fractional flow equations, including capillarity and gravity effects to the description of one-dimensional, gaswater syștem gravity drainage experiments conducted in the laboratory, and obtained good agreement between experimental and computed performance data.

Numerical methods of solving partial differential equations are not new. However, within the last decade and a half tremendous strides have been made. Southwell (31) describes the relaxation methods used before mid 40's. In 1947 Crank and Nicholson developed a practical method of numerically integrating partial differential equations of heat conduction type (32). Researchers like Frankel (33, 34 ) and DeFort (34) investigated the stability conditions and convergence rates of numerical treatment of partial differential equations (PDE).

From the early 1950's until the present, three researchers made greatly significant contributions to the numerical solution of parabolic and elliptic PDE.

Starting in 1952, Douglas, Peaceman and Rachford (3642) developed various implicit and explicit methods of solving PDE governing heat conduction, and fluid flow in one to three spatial dimensions. These investigators, as well as others, established rigorous stability and convergence criteria for various finite difference methods of solving PDE. Coates $(50,51,52,53)$ has been the leading 
reservoir technologist in applying and extending the works of Douglas, Peaceman and Rachford to multi-phase multidimensional fluid flow in porous media.

In 1954 West et al. (43) studied horizontal and linear systems produced under gas drive, neglecting capillary phenomena. They used implicit finite difference grid systems. Others investigated $(44,45)$ one dimenșional flow, including capillarity and gravity effects, and structural inclination. Sheldon et al. (46) transformed onedimensional two-phase flow equations to Lagrangian coordinates, where saturation and time are independent variables, and applied the method of characteristics in obtaining numerical solutions.

In 1953 Peaceman and Rachford (36, 39) proposed the first alternating-direction implicit (ADI) technique of solving parabolic (or unsteady-state) problems, and iterative ADI procedure of solving elliptic (or steady-state) problems. This technique reduced the machine computation time and provided a lift for expanding research on numerical simulation methods. However, the generalization of this method to three spatial dimensions was not stable for large ratio of time increment to the square of space increment. Douglas and Rachford (40) proposed a second ADI method, which, in three dimensions is a perturbation of the backward difference equation (first order correct in time). Douglas later proposed a third ADI method (47) for three space variables that is a modification of the Crank-Nicholson 
equation; consequently, it is second order correct in time. In this author's opinion, the most important single paper in numerical simulation is one by Douglas, Peaceman, and Rachford (42), in which classical finite difference treatment of two-phase, immiscible, incompressible fluid flow in porous media, in two dimensions was made by using iterative $\mathrm{ADI}$ method.

In 1965 Quan, Dranchuk, and Allada introduced an "Alternating Direction Explicit" (48, 49) -- abbreviated $\mathrm{ADE}$-- process for two dimensional two-phase systems. The following year coates et al.. (50) reported important findings in the comparison of $\mathrm{ADI}$ and $\mathrm{ADE}$ applied to various reservoir problems. They found ADI superior in accuracy but slightly slower than ADE, in contrast with earlier claims that ADI was much slower than ADE (49).

The most recent report on simulating two phase, immiscible fluid system behavior in porous media is a SPE paper by Coates et al. (8) where a three-dimensional model for two-phase, immiscible, compressible flow, including capillarity and gravity effects are treated neglecting hysteresis in relative permeability and capillary pressure. Coates also presented recent papers on the simulation of three-phase flow $(52,53)$. Excellent papers by Irby and Lamoreaux (54), Schwaube and Brand (55), Garrett (56), and Briggs and Dixon (57) are available in SPE literature on the progress and evaluation of multiphase flow simulators. 
This research project was initiated to develop a performance evaluation-prediction tool for cyclic gas movement in aquifer storage and, in particular, estimation of residual gas remaining in an aquifer-reservoir complex at abandonment. The movement of natural gas in contact with underground water is a special case of multi-phase immiscible displacement in porous media. Hence, the central problem of this dissertation is the extension of the theory of immiscible displacement to include multi-cycle drainageimbibition processes involving two compressible fluids, and to develop a mathematical simulator of the flow system.

The theory of immiscible fluid displacement in porous media is based on the fundamental concepts of relative permeability, capillarity, Darcy's Law, equation of state, and material balance. Multi-cycle drainage-imbibition processes differ from the non-cyclic displacement in that dynamic hysteresis exhibited by relative phase permeability and capillarity must be considered.

In order to save space, the following abbreviations were used in the rest of this dissertation:

$\mathrm{PC}=$ capillary pressure 


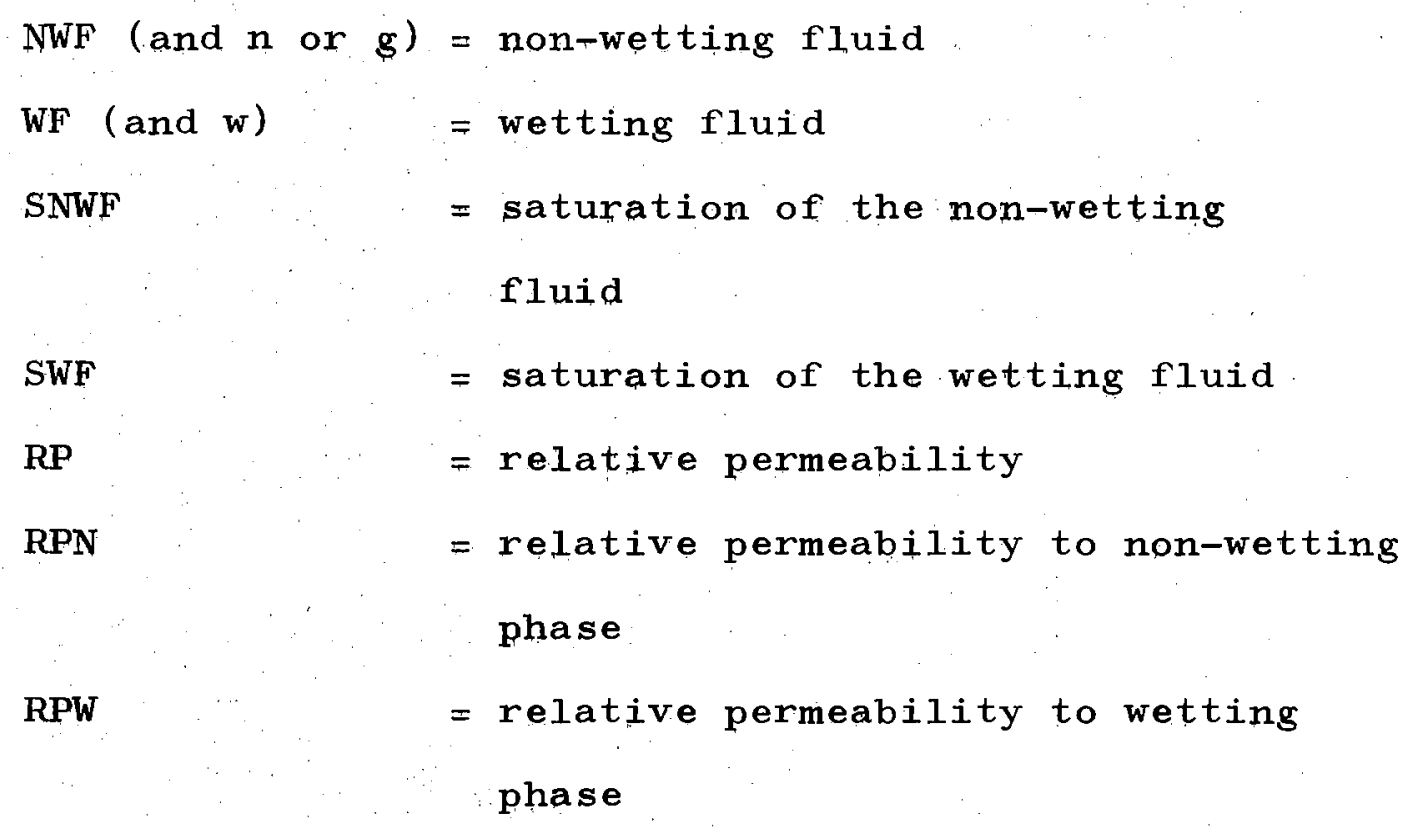

In studies of immiscible displacement, it is common practice to assume that WF saturation is everywhere decreasing or increasing. This permits the classification of the displacement process as drainage (desaturation) or imbibition, everywhere in the rock-fluid system being considered. Until the experimental verification of the existence of hysteresis in PC or RP versus phase saturation relationships, correlations based on drainage conditions alone were used in reservoir and well performance equations. Today, it is realized that the two types of displacement mechanisms are each associated with separate RP and PC characteristics, and drainage or imbibition data is used depending on the classification of the process studied. It is common practice to use imbibition data for secondary, tertiary, etc., drainage-imbibition processes,

The normal procedure is to obtain PC-saturation and RP-saturation data by testing core samples presumably 
representing the reservoir rock, or from correlations published in the literature for rock-fluid systems of similar characteristics $(9,11,14,24-28)$. These tests expose the core samples to extremes of phase saturations. A typical drainage PC or RP test is conducted until WF saturation is reduced to an apparent minimum (residual) level. Similarly, imbibition testing is started from this WF residual saturation and continued until an apparent minimum (residual) NWF saturation is reached. This type of testing establishes initial drainage-imbibition curves which form hysteresis loops in PC and RP $(9,11,24,27)$. However, in cyclical mode of operating two-phase systems, substantial, if not all, regions of the reservoir rock are not subjected to one or more phase saturation extrema, and all regions do not always experience either drainage or imbibition simultaneously. Hence, RP and PC versus WF saturation correlations based upon initial drainage-imbibition test data are inadequate for the simulation of cyclic flow. This is especially significant in gas storage reservoir-aquifer systems and in studying the behavior of wells subject to water coning. Cyclic test methods and equipment need new development in order to obtain dynamic hysteresis data on porous solid samples. In fact, regular drainage and imbibition test methods and equipment are by no means perfect. However, it seems quite appropriate, at first, to investigate the sensitivity of a numerical simulator to model hysteretic PC and RP data. Parametric simulation studies of hypothetical 
systems representing typical reservoirs can provide valuable information as to the detail required. With this in mind, it was decided to conceptualize dynamic hysteresis models for PC and RP behavior based on available data and intuition, and to develop a mathematical simulator incorporating these systems. If rigorous representation of dynamic hysteresis in RP and PC is found to affect significantly the accuracy of numerically computed pressure and saturation distributions, extensive laboratory research will be needed to support, modify, or replace the models conceptualized and used in this dissertation.

The convention of this dissertation concerning hysteretic RP and PC models is presented in Chapter IV. The development, and the application of the hysteretic flow simulator are covered in Chapters V and VI, respectively. 


\section{CHAPTER IV}

\section{GENERATION OF HYSTERETIC CAPILLARY PRESSURE}

\section{AND RELATIVE PERMEABILITY CURVES}

In this dissertation generalized semi-empirical relations, based on the theoretical treatment of the fundamental factors controlling the quantitative features of $P C$ and $R P$, were used to generate cyclic drainage-imbibition data. Since the parametric analysis of the simulator sensitivity to the hysteresis in RP and PC was the secondary object of this work, the only requirement in using generalized relations is that they must generate curves spanning the usual ranges of the typical experimental $R P$ and $P C$ data for porous solids. Furthermore, generalized expressions allow the employment of rock-fluid properties appearing in them as parameters for sensitivity analysis.

\section{Hysteretic PC Model}

The theory of immiscible fluid displacement in porous media defines the capillary pressure as:

The pressure difference that occurs between the mobile interconnected masses of the two fluids in question, which is associated with the saturation at which occurs, even though the relation is not altogether permanent. 58

This definition leads to a model of capillary behavior 
consisting of lines drawn through points representing empirical PC-saturation data from core samples. Various methods of measuring $P C$ and the bulk phase saturations are described in the literature $(9,13,16,17)$. These experimental points are determined at apparent equilibria reached after the cessation of hydrodynamic flow; that is, after the bulk WF flow into or out of the porous solid sample has stopped. This model capillary behavior is based on the following assumptions: (1) net mass transfer across a phase boundary is negligible and (2) pellicular moisture does not provide a path for bulk liquid transfer.

Morrow and Harris (17) used a modified suction potential technique to study the cyclic relationships between PC and the moisture content for a porous mass. They proposed a model of hysteretic capillary behavior in which the above two conditions are assumed. Figure 1 shows the general form of the principal relationships between PC and bulk SWF obtained by Morrow-Harris. Three principal curves can be named: (1) primary drainage $D_{O}$, representing the initial desaturation from the condition of complete initial saturation by the WF; (2) pendular imbibition $I_{O}$, delineating imbibition from the residual SWF; and (3) secondary drainage D, portraying desaturation from the residual SNWF. In this dissertation, the curves $D_{O}$ and $I_{O}$ will be called Bounding Drainage and Bounding Imbibition Curves for capillarity.

Using consolidated ceramic tiles and unconsolidated ground glass initially saturated by boiling in water, 
Morrow and Harris reproduced saturation cycles of type $I_{O} D$ 。 After resaturation by boiling, the curve $D_{\circ}$ and subsequent cycles could also be reproduced. Scanning curves were obtained by these investigators when a drainage or imbibition process was reversed before it reached the corresponding residual saturation, as illustrated in Figure 1.

Multi-cycle capillarity tests, patterned after the Morrow-Harris method, involving reservoir rock and fluids have not been reported in the literature. $\operatorname{Comer}^{+} \mathrm{s}(11,15)$ unpublished cyclic PC data on consolidated and unconsolidated reservoir rock samples is shown in Figures 2 and 3 . Sample properties are also given. Core samples were taken. from two sandstone formations of Cambrian Period, used as gas storage reservoirs. Three hysteresis cycles, bounded by phase saturation extrema, are clearly discerned on Figures 2 and 3 . Within the accuracy of the experiments, Comer's data support the findings of Morrow-Harris concerning the loci $D_{0}-I_{0}-D$ of the empirical PC data. Hence, reservoir rock-fluid systems exposed to saturation extrema can be expected to behave as shown in Figure 1.

Figure 1 also defines the hysteretic capillarity model used in this dissertation. The loci of the bounding drainage and imbibition curves are called "capillary pressure or capillarity envelope." Intermediate scanning capillarity data are represented by scanning drainage or scanning imbibition curves. Only one type of intermediate hysteresis loop is shown in Figure 1. The loop ABCEA contains parts 


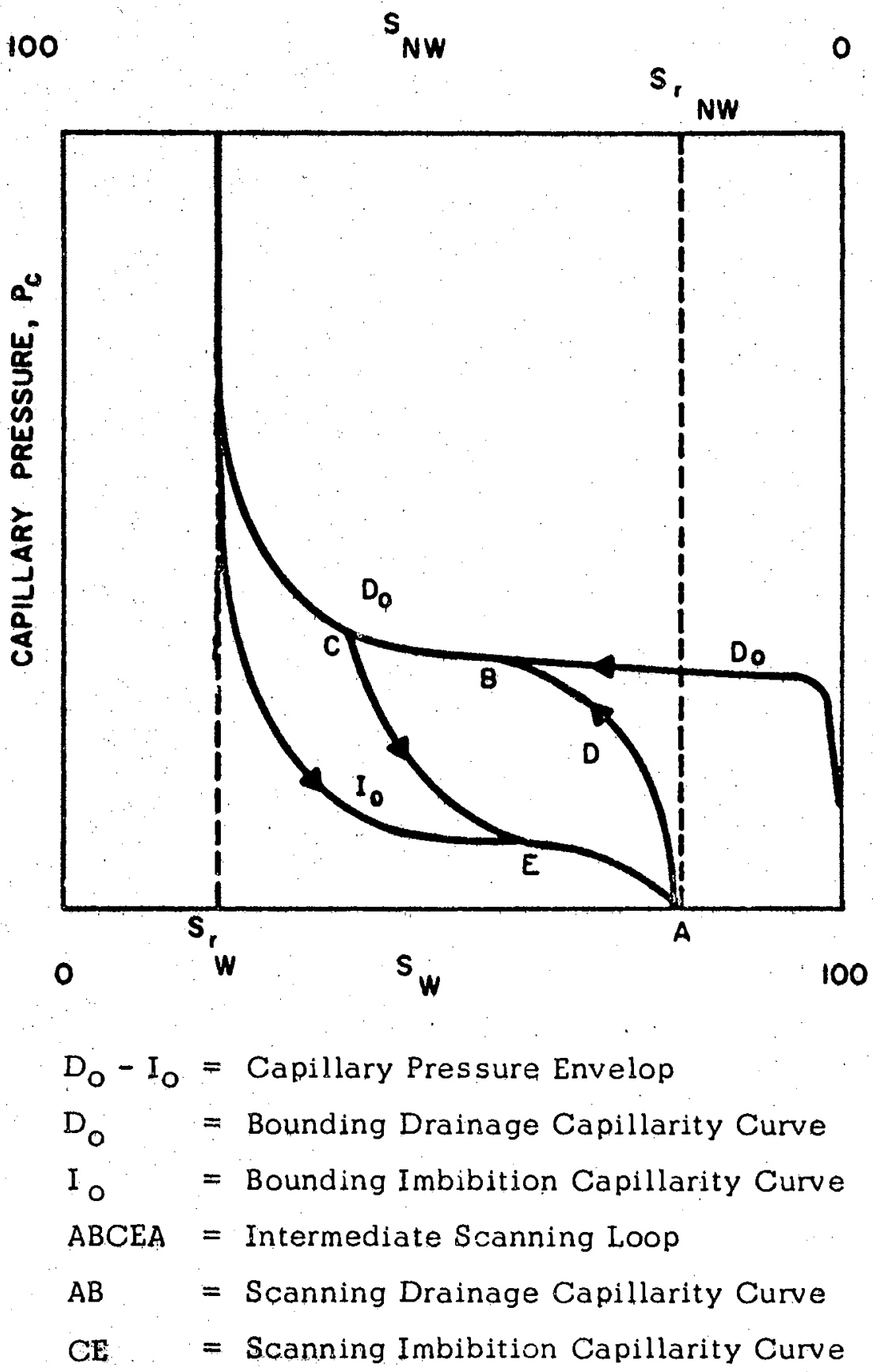

Figure 1. Typical Relationships Between Capillary Pressure and Saturations of Wetting and Non-Wetting Phases 


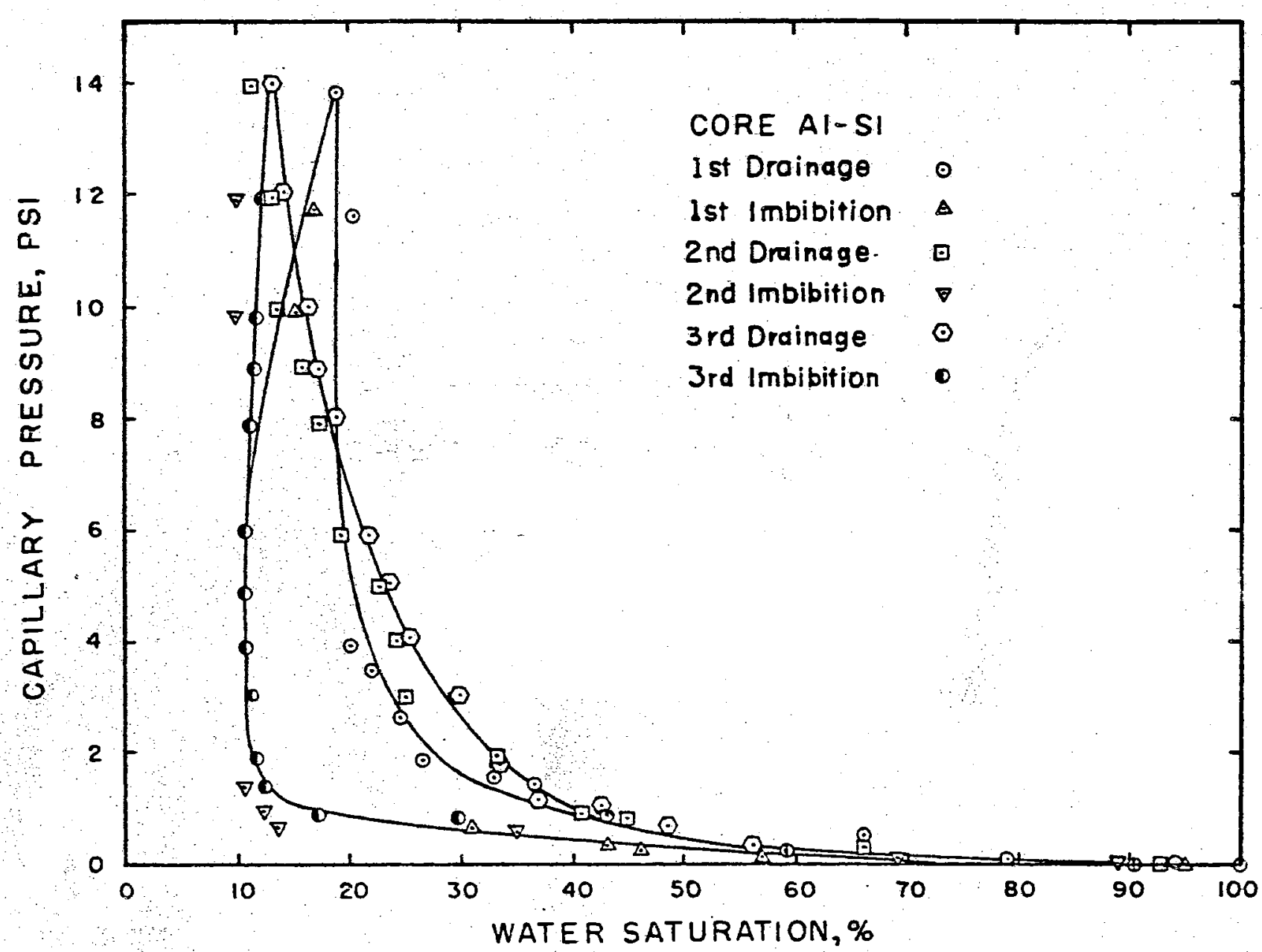

Figure 2. Cyclic Capillary Pressure, Core A1-S1 


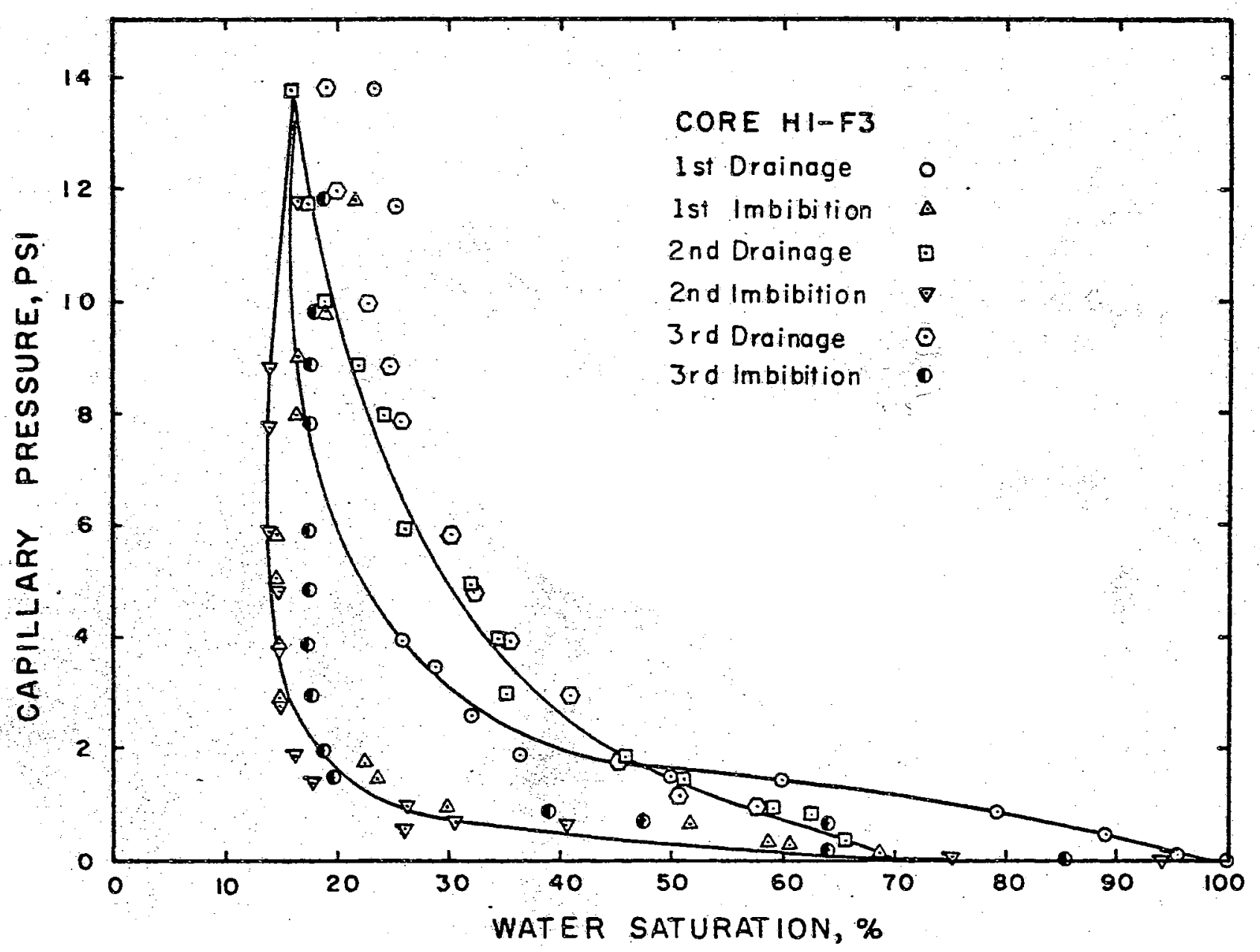

Figure 3. Cyclic Capillary Pressure, Core H1-F3 
of the bounding drainage and imbibition curves. A secondary drainage process can start at any point $A$ on the bounding imbibition curve $I_{0}$; it is not restricted to the point where $S_{n}=S_{r_{n}}$. The extreme situation is one that produces a scanning loop that never "touches" the bounding curves. The model capillary behavior depicted in Figures 1 through 3 implies complete desaturation of the wetting phase during the initial drainage process. However, a primary drainage process can be stopped at any stage before bulk WF is completely isolated, and imbibition started with $S_{w}>S_{r w}$. As a matter of fact, it is possible to go through several drainage-imbibition cycles before residual SWF is reached. Hence, the static capillarity envelope defined in Figure 1 for a given sample is not unique. In reality, there are virtually an infinite number of families of hysteresis loops, each confined within an envelope characterized by the branching point of the bounding imbibition curve from the drainage curve $D_{0}$. In order to account for this behavior a "growing" or dynamic hysteresis envelope concept, illustrated in Figure 4, was used in this dissertation. According to this concept, the initial hysteresis loop $A B C$ forms the capillarity envelope if the SWF for all subsequent cycles remains higher than the saturation attained at the end of the initial drainage. Otherwise, the envelope grows in steps as shown in Figure 4.

The concept of the dynamic capillarity envelope includes a fixed locus for the bounding drainage data $\mathrm{AH}$, a 


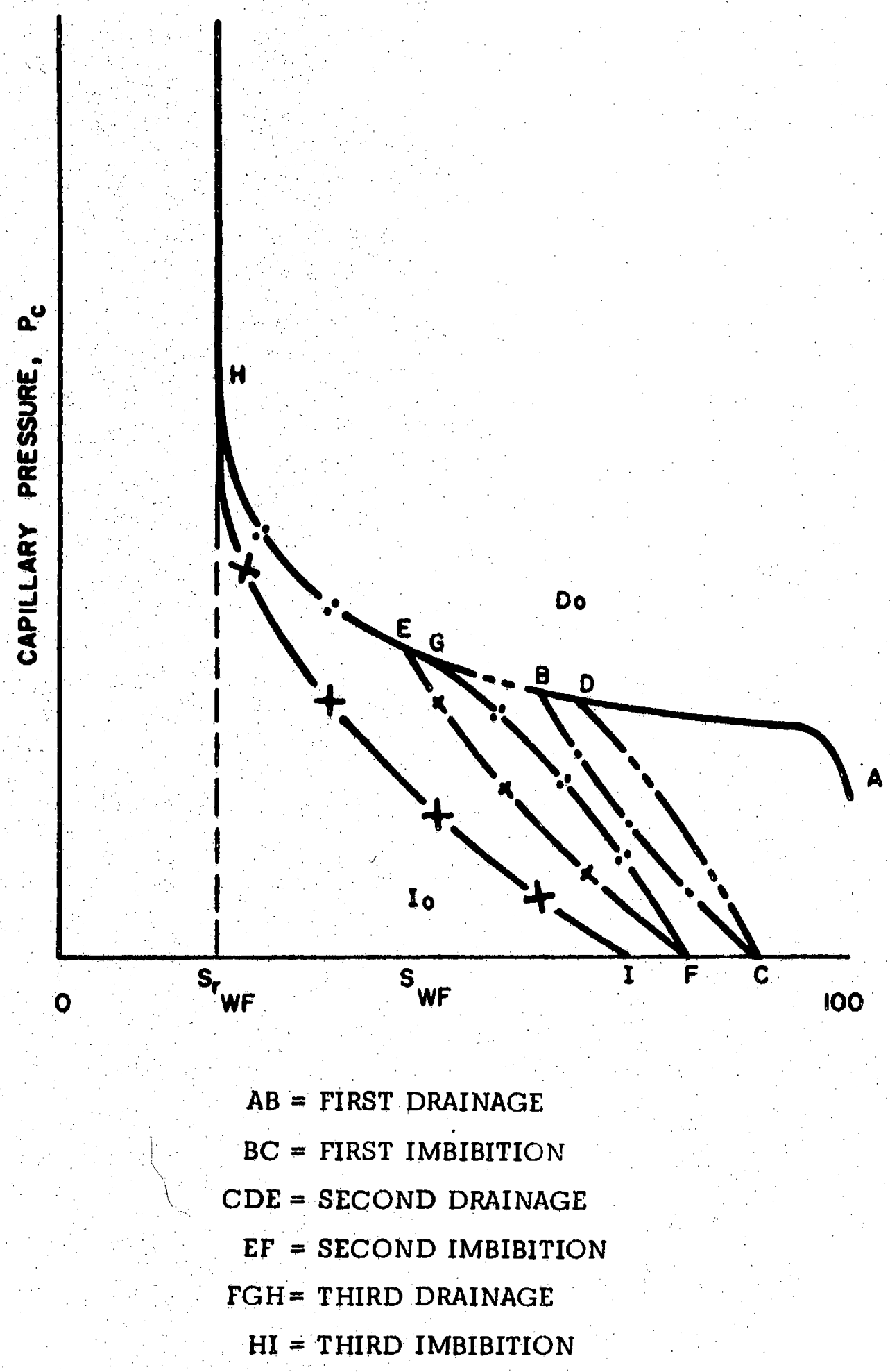

Figure 4. Growth in Capillary Pressure Envelope 
shifting locus for the bounding imbibition data (BC to EF to HI), and a relationship between the maximum and residual SWF. One cannot compute from first principles the quantity of NWF which will be left behind an advancing WF front in a given porous solid. However, several papers have been published relating, by experimental results, the initial SNWF saturation to trapped $\operatorname{SNWF}(28,59-63)$. The model capillary behavior of this dissertation relates the residual SNWF to historical maximum SNWF.

\section{Hysteretic RP Model}

Naar, Wygal, and Henderson (64) reported extensive experimental work in 1962 , which showed that consolidated rocks and unconsolidated porous media exhibit different imbibition flow behavior. Figure 5, reproduced from their original article, exemplifies their findings. They noted similarities in the static capillary equilibrium curves for consolidated sands and unconsolidated glass spheres, which support the findings of the authors (see Figures 2 and 3 ). RP testing of the same samples with oil and air, produced the usual hysteresis in RPN (air) and RPW (oil) for consolidated samples, shown in Figure 5a; however, the unexpected contrary behavior, shown in Figure 5b, held for the unconsolidated samples. These investigators also noted that:

A significant difference was also observed when drainage and imbibition processes were repeated: (1) when after imbibition a WF is drained and then imbibed in a consolidated rock, the RP behavior retraces the imbibition curves only, (2) when these processes are applied to an 

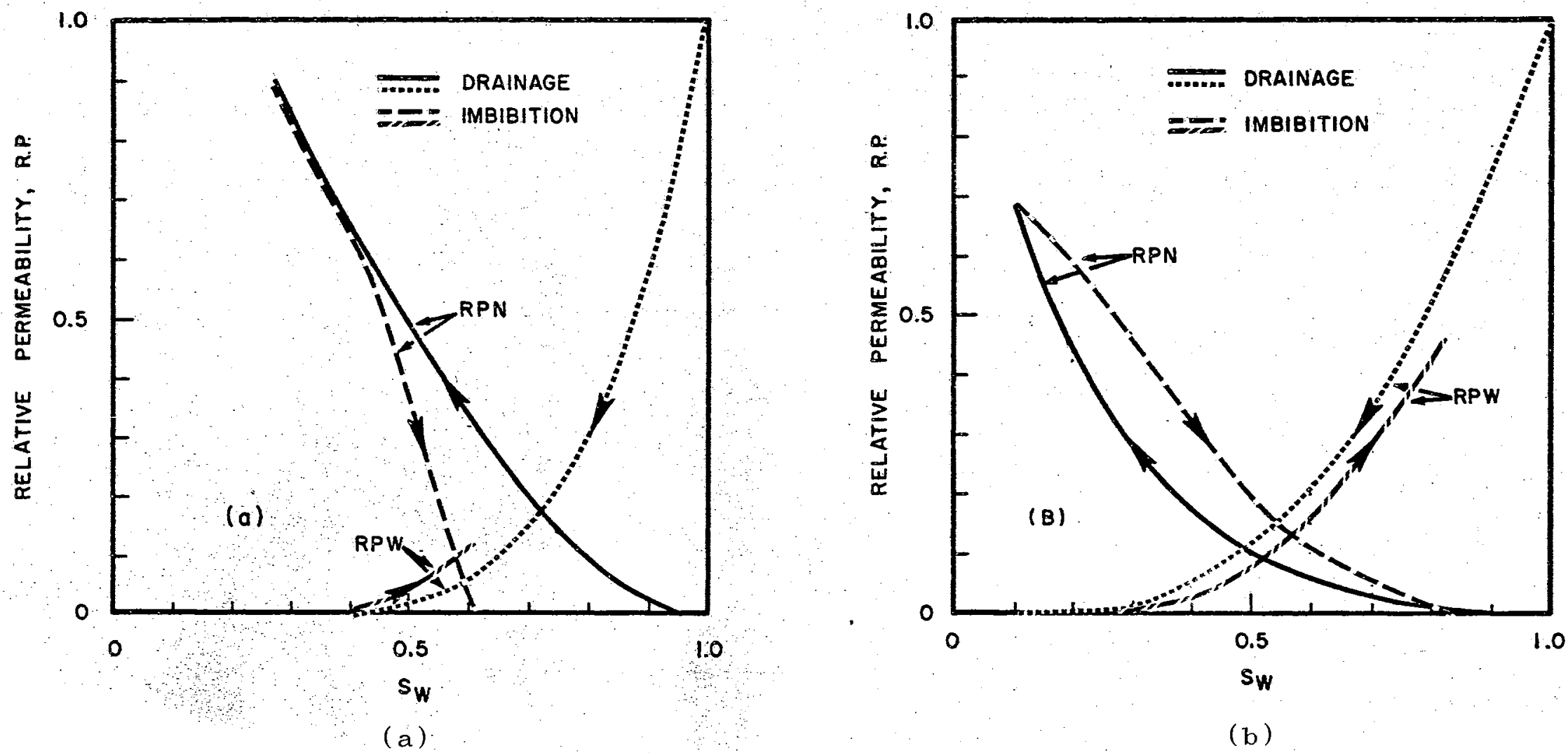

(b)

Figure 5. Relative Permeability Curves for (a) Consolidated Sands (b) Unconsolidated Glass Spheres (Reproduced From Naar-Henderson (64) Original Article) 
unconsolidated sand, the flow behavior retraces in succession imbibition and drainage RP curves.

These statements are in conflict with authors' cyclic RP test data on samples $A 1-S 1$ and H1-F3, presented in Figures 6 and 7. Capillarity data for these samples were shown in Figures 2 and 3. Sample A1-S1 is an unconsolidated sandstone whose RPN behavior supports the second statement made by Naar et al., and whose RPW behavior rejects it. Similarly, the RPN and RPW behaviors of the sample H1-F 3 contradict fully the first statement of Naar, et al. As a matter of fact, according to data presented in Figures 6 and 7, the RP behavior of consolidated and unconsolidated sands are similar. Extensive unreported cyclic test data on other samples from consolidated formations, used by the author and his co-warkers as storage reservoirs, exhibited the same behavior as in Figure 7 .

A discussion of the contrasts between the data results of Comer and Naar, et al., is beyond the scope of this dissertation for it would require rather lengthy descriptions of experimental environment and techniques underlying the data. Their findings were briefly covered above in order to emphasize two key points: (1) if one treats core data like those shown in Figures 5, 6, and 7, as model data, one is beset with the absence of agreement on the shapes of the hysteresis loops, absence of intermediate scanning data, and different hydrostatic versus hydrodynamic residual saturations, and (2) extensive laboratory research is needed to isolate true RP behavior from any abnormalities caused by 


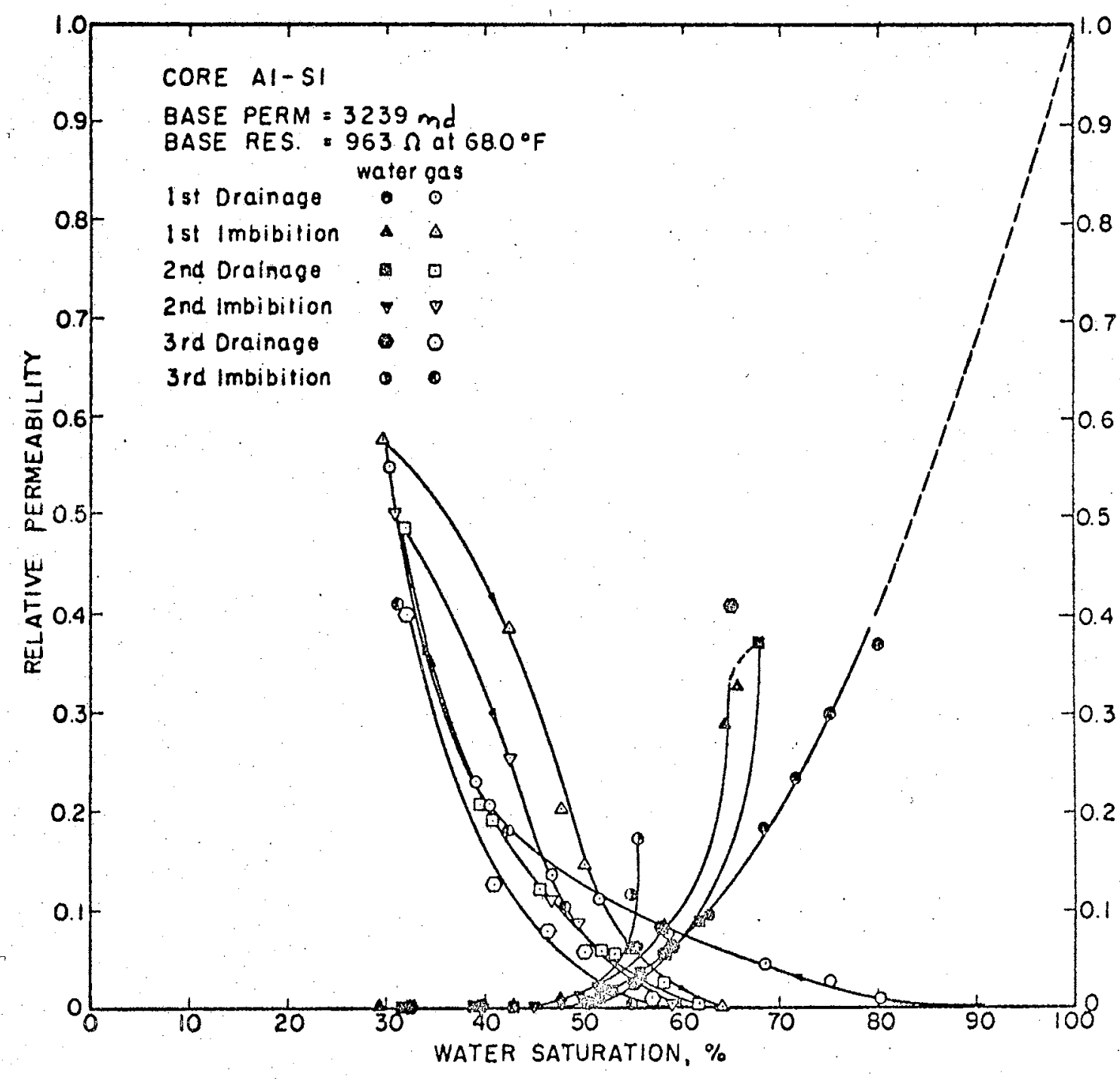

Figure 6. Relative Permeability, Core A1-S1 


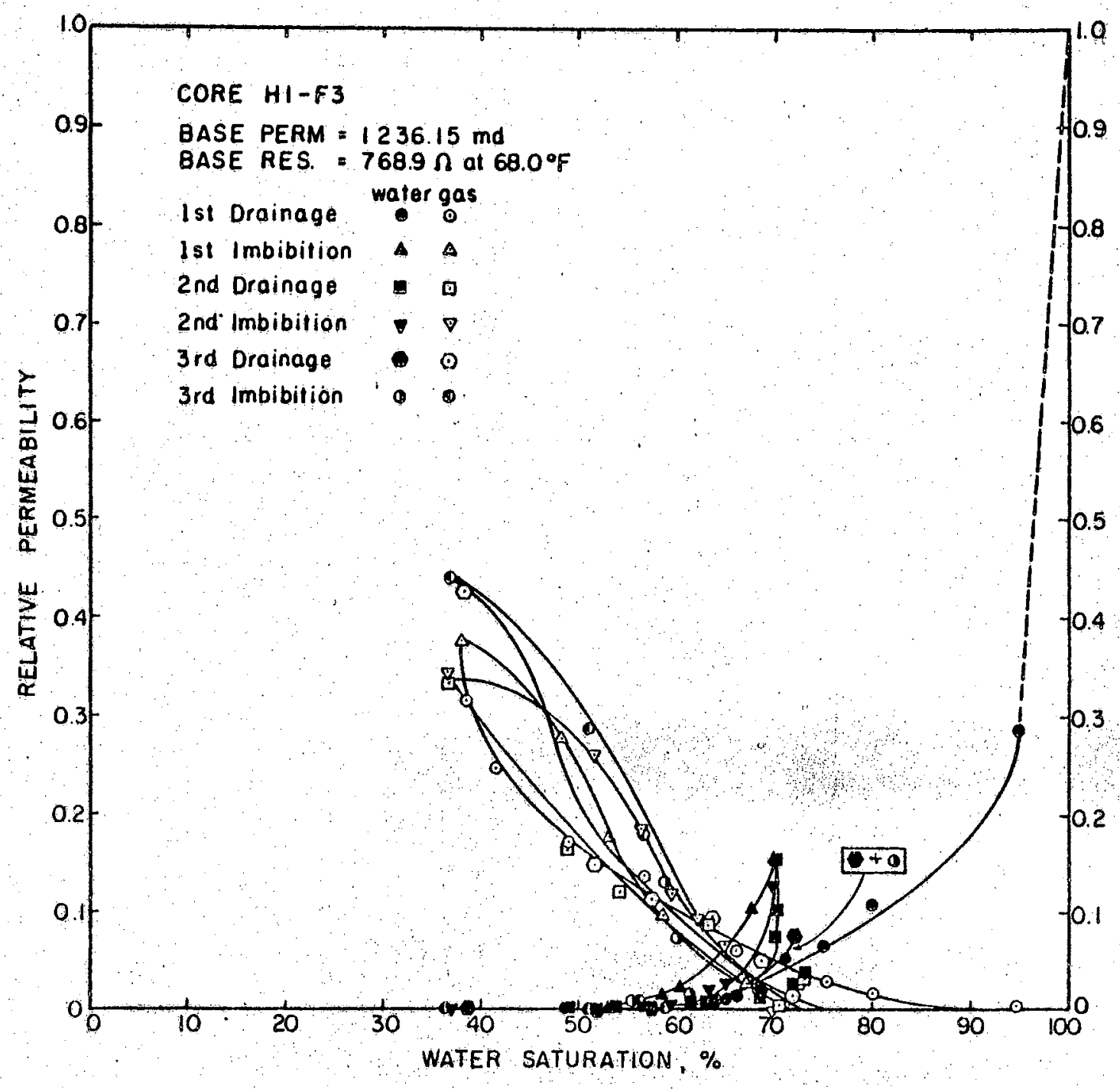

Figure 7. Relative Permeability, Core H1-F3 
specific experimental techniques. For example, RP test procedures reported in the literature use the same inlet to the core sample during drainage and imbibition testing. After drainage testing is completed, the core samples should be rotated or the inlet-outlet positions switched in order to better simulate prototype flow conditions.

Various models of porous rock have been proposed to develop theoretical expressions for $R P$. These range from parallel-capillary models to randomly interconnected capillary models; i.e., Kozeny-Carman (65), Rose (66, 67), Marsha11 (68), Wy11ie-Gardner (69), Naar-Henderson (70), Bolt (71) models. It was found that, with certain modifications, the expressions proposed by Wyllie-Gardner (69) for generating the primary drainage RP, and expressions proposed by Naar-Henderson (70) for pendular imbibition RP could be generalized to map the usual domain of this data. The concepts of a dynamic hysteresis envelope consisting of bounding drainage and bounding imbibition curves, and intermediate scanning curves, were extended to the realm of $R P$. It was assumed that imbibition RPN would be smaller than drainage RPN for a given saturation of the bulk WF. The converse was assumed for RPW.

Semi-Empirical Relations for Hysteretic RP and PC

Considering a set [ $\left.S^{*}\right]$ of saturation states consisting of laboratory measured properties of the rock-two phase 
fluid system, such as saturation extrema (critical and extreme residual saturations), and any saturation milestone in the saturation history which can be used in semi-empirical equations describing the loci of hysteretic RP and PC:

$$
\begin{aligned}
& \mathrm{S}_{\mathrm{n}} \quad=\text { current } \mathrm{SNWF} \\
& S_{0 n}=\text { critical } \mathrm{SNWF} \\
& \mathrm{S}_{x \mathrm{n}}=\text { residual } \mathrm{SNWF} \\
& S_{r W}=\text { residual } \mathrm{SWF} \\
& S_{m n}=1-S_{r w}=\text { Upper limit of SNWF } \\
& \mathrm{S}_{\mathrm{h} \text { an }}=\mathrm{SNWF} \text { reached when a given drainage process is } \\
& \text { terminated and imbibition started } \\
& S_{h n}=\operatorname{maximum} S_{h_{s}} \text { considering the saturation history } \\
& \text { of the system, } S_{n n} \leq S_{n n} \\
& S_{1 s n}=S N W F \text { reached when a given imbibition process is } \\
& \text { terminated and drainage started, } \geq S_{n n} \\
& S_{1_{n}}=\text { minimum } S_{1, n}, \geq S_{n}, \text { considering the saturation } \\
& \text { history }
\end{aligned}
$$

and defining transformed saturations,

$$
\begin{aligned}
& S_{1} \equiv \frac{S_{n n}-S_{n}}{S_{m n}} ; \quad S_{2} \equiv \frac{S_{m n}-S_{n}}{S_{n n}-S_{0 n}} ; \quad S_{3} \equiv \frac{S_{n}-S_{n n}}{S_{n n}-S_{n n}} \\
& S_{4} \equiv \frac{S_{n n}-S_{n}}{S_{n n}-S_{n n}} ; \quad S_{5} \equiv \frac{S_{n}-S_{18 n}}{S_{n n}-S_{18 n}} ; \quad S_{5} \equiv \frac{S_{n=n}-S_{n}}{S_{n s}-S_{n n}}
\end{aligned}
$$

and letting:

$$
\begin{aligned}
& \text { UNDR }=\text { Bounding Drainage } \mathrm{RPN} \\
& \text { UWDR }=\text { Bounding Drainage RPW } \\
& \mathrm{PCDR}=\text { Bounding Drainage } \mathrm{PC}
\end{aligned}
$$




$$
\begin{aligned}
& \text { UNIM }=\text { Bounding Imbibition RPN } \\
& \text { UWIM }=\text { Bounding Imbibition RPW } \\
& \text { PCIM }=\text { Bounding Imbibition PC }
\end{aligned}
$$

semi-empirical relations were developed for $R P$ and $P C$ as follows.

Bounding Drainage Equations

$$
\begin{aligned}
& \operatorname{RPN} \equiv \operatorname{UNDR}=\left\{\begin{array}{l}
F_{n}\left(1-S_{2}\right)^{\lambda_{n}} ; S_{0 n}<S_{n} \leq S_{n n} \\
0 ; 0 \leq S_{n} \leq S_{\theta n} \cdots
\end{array}\right. \\
& \mathrm{RPW} \equiv \mathrm{UWDR}=\mathrm{F}_{\mathrm{w}} \mathrm{S}_{1} \lambda_{w} ; 0 \leq \mathrm{S}_{\mathrm{n}} \leq \mathrm{S}_{\mathrm{mn}} \\
& P C \equiv P C D R=f\left(S_{w} \text { or } S_{n}\right)
\end{aligned}
$$

where,

$$
\begin{aligned}
& \lambda_{n}=\text { drainage interference exponent for NWF } \\
& \lambda_{w}=\text { drainage interference exponent for WF } \\
& F_{n}=\text { drainage interference coefficient for NWF } \\
& F_{w}=\text { drainage interference coefficient for WF. }
\end{aligned}
$$

Given set $\left[S^{*}\right], \lambda$, and $F$ control the shapes of UNDR and UWDR. Mutual interference in simultaneous flow of NWF and WF is responsible for the fact that, at any saturation $S_{n}>0, R P^{\prime} s$ of the two phases do not add up to unity; hence, the coinage of the labels "interference exponent" and "interference coefficient." $F_{n}$ and $F_{w}$ were used as multipliers to shift the RP curves about the pivotal saturations $S_{e}$ and $S_{s}$, respectively. Figures $8 a$ and $8 b$ show UNDR and UNIM generated by Equations (6) and (7), for 


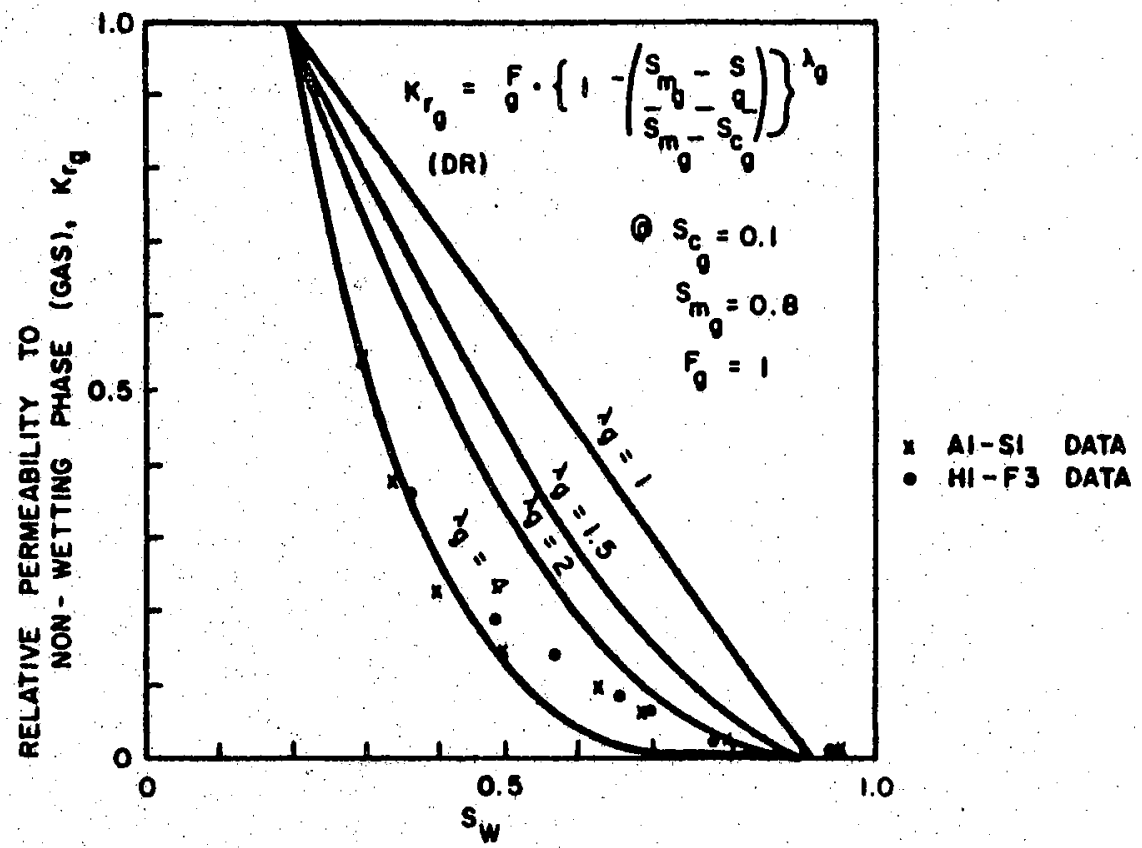

(a)

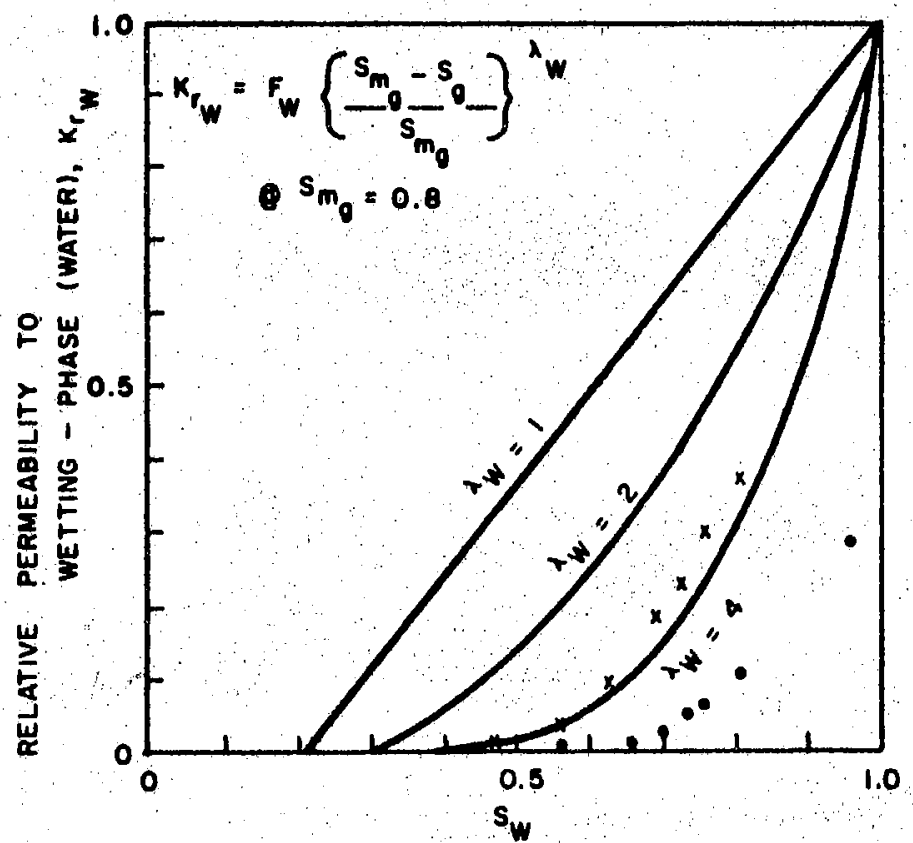

(b)

Figure 8. (a) Bounding Drainage RPN Curves Generated by Equation (6)

(b) Bounding Drainage RPW Curves Generated by Equation (7) 
various values of $\lambda$. As $\lambda_{n}$ and $\lambda_{n}$ increase, $R P$ values at a given saturation $S_{n}$ decline. Hence, higher values of $\lambda$ represent high order interference. Actual UNDR values from core samples $\mathrm{A} 1-\mathrm{S} 1$ and $\mathrm{H} 1-\mathrm{F} 3$ are plotted as dotted lines on Figures $8 \mathrm{a}$ and $8 \mathrm{~b}$ for comparison. The relationship between PCDR and $S$ (Equation 8 ) was represented in the numerical simulator in tabular form.

Equations (6) and (7) were obtained by modifying and generalizing Wyllie-Gardner (69) statistical-capillaric expressions for RP

$$
\begin{aligned}
& R P W=\left(S_{w} *\right)^{4} ; S_{w} * \equiv \frac{S_{y}-S_{w w}}{1-S_{r w}} \\
& R P N=\left(1-S_{w} *^{2}\right)\left(1-S_{w}\right)^{2}
\end{aligned}
$$

These equations are based on over-simplifying assumptions, such as linearity of the relationship between $1 / \mathrm{P}_{c}{ }^{2}$ and $S_{k} *$, and the condition of $R P N=1$ for $S_{w}=S_{r}$. The author's experience has always been that $\operatorname{RPN}\left(S_{\mathrm{N}}\right)<1$.

\section{Bounding Imbibition Equations}

The first imbibition process may start at any $S_{w}$, larger than or equal to $S_{p w}$. Therefore, the first imbibition RP and PC curves constitute the bounding imbibition curves (UNIM, UWIM, PCIM), as long as subsequent drainage processes are terminated at or before the maximum SNWF reached at the end of the initial drainage, Otherwise, the loci UNIM, UWIM, and PCIM will shift as $S_{h n}$ value associated 
with each drainage process increases. The following relationships were developed for the dynamic UNIM, UWIM, and PC IM.

$$
\begin{aligned}
& \mathrm{UNIM} \equiv \mathrm{UNDR} \cdot \mathrm{S}_{3} \mathrm{Wn}_{\mathrm{n}}=\mathrm{F}_{\mathrm{n}}\left(1-\mathrm{S}_{2}\right)^{\lambda_{\mathrm{n}}}\left(\mathrm{S}_{3}\right)^{W_{n}} \\
& \mathrm{UWIM} \equiv \mathrm{E}_{\mathrm{w}} \quad\left\{\mathrm{UWDR}+(1-\operatorname{UNDR})\left(\mathrm{S}_{4}\right)^{W_{w}}\right\} \\
& =E_{w}\left\{F_{w} S_{1} \lambda_{w}\left(1-S_{4}\right)^{\omega_{w}}+S_{4} w_{w}\right\} \\
& \operatorname{PC} I M \equiv \operatorname{PCDR} \cdot\left(S_{3}\right)^{w_{\sigma}}
\end{aligned}
$$

where

$$
\begin{aligned}
& E_{w}=\text { imbibition interference coefficient for WF } \\
& w_{n}=\text { imbibition interference exponent for NWF } \\
& w_{w}=\text { imbibition interference exponent for } W F \\
& w_{c}=\text { imbibition exponent for PC }
\end{aligned}
$$

when $S_{n n}=S_{m n}$, Equations 11, 12, and 13 give the loci of the limiting UNIM, UWIM, and PCIM. Figure 9 shows how PCIM values vary as a function of $\omega_{0}$ for various values of $S_{h_{g}}$.

Equations developed for the intermediate drainage and intermediate imbibition RP and PC are given in Appendix A. 


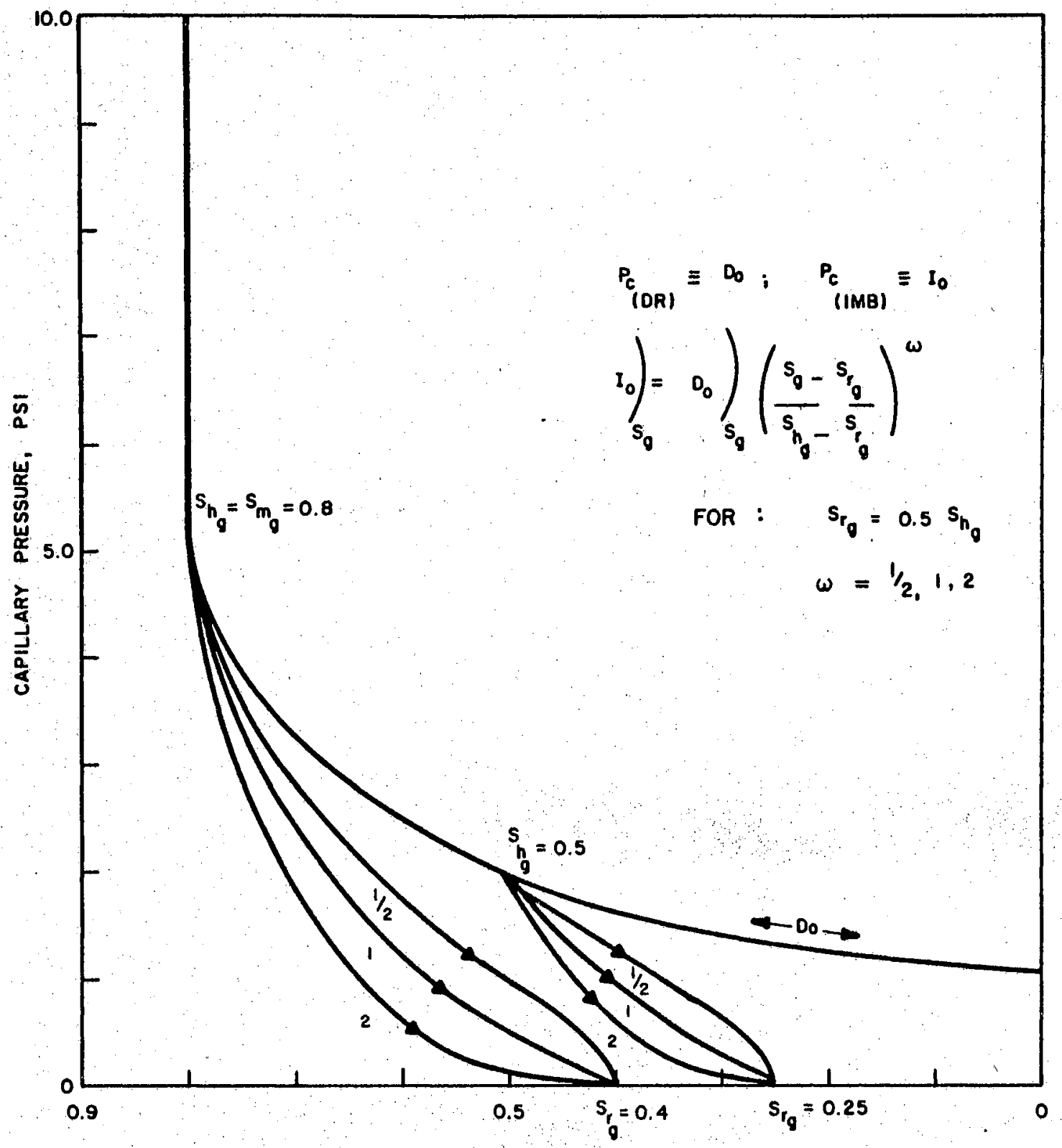

Figure 9. Various Paths for Bounding Imbibition Capillarity Curves Generated by Equation (13) 


\section{CHAPTER V}

\section{DESCRIPTION OF THE NUMERICAL MODEL}

The flow of two immiscible, compressible fluids through porous media, governed by interacting viscouscapillary-gravitational forces, can be represented by the following simultaneous set of second-order, non-linear, partial differential equations:

$$
\begin{aligned}
& \nabla \cdot\left[\left(k_{r} \rho_{g} / \mu B\right)_{n}\left(K \cdot \Phi_{n}\right)\right]+\left(q_{g}\right)_{n}= \\
& \varphi\left[\left(-S^{\prime} / B\right)_{n}\left(\rho_{B} \partial \Phi_{n} / \partial t-\rho_{B} \partial \partial \Phi_{w} / \partial t\right)\right. \\
& \left.+(1-S) \rho_{B n}\left(1 / B_{n}\right)^{\prime} \partial \Phi_{n} / \partial t\right] \\
& \nabla \cdot\left[\left(\mathbf{k}_{g} \rho_{\mathrm{g}} / \mathcal{B}\right)_{W}\left(\mathrm{~K} \cdot \nabla \Phi_{W}\right)\right]+\left(\mathrm{q}_{\mathrm{g}}\right)_{W}= \\
& \varphi\left[\left(S^{\prime} / B_{w}\left(\rho_{\mathrm{B} n} \partial \Phi_{\mathrm{n}} / \partial t-\rho_{\mathrm{B}} \partial \Phi_{\mathrm{w}} / \partial t\right)\right.\right. \\
& \left.+\operatorname{Sp}_{\mathrm{w}}\left(1 / B_{w}\right)^{\prime} \partial \Phi_{w} / \partial t\right]
\end{aligned}
$$

These equations are derived by combining Darcy's Law for each phase $f=(n, w)$

$$
\bar{V}_{p}=-K\left(k_{g} / \mu\right), \rho_{B} \nabla_{p}
$$

with an equation of continuity for each component,

$$
-\nabla \cdot\left(\rho_{g} \bar{V}\right)_{i}=\varphi \partial\left(\rho_{g} S\right)_{t} / \partial t
$$


where the flow potential $\Phi_{f}$ is defined by,

$$
\Phi_{f} \equiv \int_{P_{0}}^{\rho_{f}} \frac{d p}{\rho_{s f}(P)}-D .
$$

Equations ( $14 \mathrm{a} \& \mathrm{~b}$ ) are coupled through the capillary pressure-saturation relationship:

$$
\begin{aligned}
P_{0} & =P_{n}-P_{w}=\rho_{s n} \Phi_{n}-\rho_{s w} \Phi_{w} \\
& =P_{0}\left([S *],\left[C^{*}\right]\right) .
\end{aligned}
$$

The formation volume factor B replaces the specific weight terms, $p_{s}$, from the continuity equations:

$$
B=\frac{\rho_{s}(\text { reservoir conditions })}{\rho_{s}(\text { standard conditions })} .
$$

The differential model stated in Equations (14a \& b) was expressed in implicit finite difference form,

$$
\begin{gathered}
\delta \underline{T} \delta \bar{\Phi}+\bar{Q}=\underline{D} \Delta_{t} \Phi \\
\underline{T} \equiv\left[\begin{array}{rr}
T G & 0 \\
0 & T W
\end{array}\right] ; \bar{\Phi} \equiv\left[\begin{array}{c}
\Phi_{n} \\
\Phi_{w}
\end{array}\right] ; \quad \underline{D} \equiv\left[\begin{array}{ll}
G 2 & H 2 \\
G 1 & H 1
\end{array}\right] ; \quad \bar{Q} \equiv\left[\begin{array}{l}
Q_{n} \\
Q_{N}
\end{array}\right]
\end{gathered}
$$

and solved simultaneously by the three-dimensional DouglasRachford alternating-direction-implicit (ADI) iterative procedure (40). The difference notation and G1, G2, H1, and H2 are defined in Appendix B. The potential $\Phi$ on the lefthand side of Equation (20) is understood to apply at the new time $t_{\sharp+1}$. Transmissibilities were defined by:

$$
\mathrm{T}_{\mathrm{x}}=\mathrm{k}_{\mathrm{x}} \mathrm{A}(\Delta \mathrm{Y} \Delta \mathrm{Z} / \Delta \mathrm{X}) ; \mathrm{T}_{\mathrm{y}}=\mathrm{k}_{\mathrm{y}} \mathrm{A}(\Delta \mathrm{X} \Delta \mathrm{Z} / \Delta \mathrm{Y})
$$




$$
\mathrm{T}_{z}=\mathrm{k}_{\mathrm{z}} \mathrm{A}(\Delta \mathrm{X} \Delta \mathrm{Y} / \Delta \mathrm{Z}) ; \mathrm{A}=\left(\mathrm{k}_{\mathrm{r}} \rho_{\mathrm{s}} / \mu \mathrm{B}\right)_{\mathrm{s}} .
$$

The application of Douglas-Rachford ADI technique to the solution of the partial differential equations (14a \& b) is described in Appendix B; hence, it will not be detailed in this chapter. Further description of the numerical simulator will be limited to computational techniques used to incorporate RP and PC hysteresis into the iterative ADI procedure. ADI techniques have been initially developed for heat conduction and single phase mass flow studies, where the conductivity or transmissibility is not dependent on saturation. In multi-phase flow simulation with implicit difference models, it is customary to evaluate the transmissibilities at the old time $t_{n}$ and use them for the new time $t_{m+1}$ solution of the phase potentials. The dependency of the transmissibilities on phase saturations and potentials preclude the use of very large time steps.

Table look-up and interpolation techniques were used to update potential dependent properties for each potential iterate. The updating of the saturation derivative ( $\left.S^{\prime}\right)$ for use in succeding iterations at a given time step, and the updating of saturation dependent properties (RPN, RPW, PC) required a special algorithm since these properties are functions of the set of saturation states [S*]. An algorithm, named HYSTRACK, was developed as an integral part of the numerical simulator to: 1) keep track of the saturation history of any specified region of the simulated system and 2) select the appropriate RP and PC equations for updating 
these variables for use in the next time step. HYSTRACK and a simplified version called SEMHYSTRACK are described in Appendix C.

Boundary conditions for the numerical simulator consisted of: 1) explicit specification of the sink (source) terms $Q_{n}$ and $Q_{N}$, or computation from specified values of terminal flow pressures and dynamic block pressures, 2) sealed external boundaries characterized by the vanishing transmissibilities normal to the boundary, and 3) open external boundaries treated in this work by the well-known method of Carter-Tracy (72).

The following closure criteria were applied: 1) incremental material balance for each phase less than a specified tolerance, 2) maximum saturation change in a grid block less than a specified tolerance. In addition to the closure criteria, cumulative phase material balances were used to check the "correctness" of the solution, as it marched through the simulation time domain.

\section{Partial Integration of Equations of Two-Phase Flow}

Various schemes have been proposed to reduce the threedimensional problems to lower dimensions. The savings in memory storage and computer program run time are the principal motivating forces in using two-dimensional simulators to study three-dimensional flow problems. However, twodimensional simulators with pseudo-three-dimensional 
features must be used with great caution. The degree of vertical continuity of rock properties and the thickness of the reservoir rock are the controlling variables in deciding for or against the dimensional simplification.

For thin reservoirs the dip-normal component of the viscous force is nearly zero; hence, the dip-normal distribution of the fluid phases in a block depends upon the degree of vertical segregation. Two limiting cases may occur. If the rate of phase redistribution toward a capillary-gravity equilibrium configuration within a dipnormal column of fluid is high as compared to the rate of areal advance of saturation fronts, vertical equilibrium obtains. The opposite extreme is characterized by the absence of any dip-normal saturation gradient.

For thick reservoirs with good vertical continuity of rock properties, vertical equilibrium, or near equilibrium, may still obtain, if the flow rates are low and if the reservoir thickness is small relative to its areal dimensions. Otherwise, dip-normal viscous force gradients cannot be neglected.

When the capillarity of rock-fluid system is large, the NWF to WF transition zones are significant; hence, saturation and relative permeability distributions become uniform in the dip-normal direction as the reservoir thickness decreases. For low capillary pressures, usually exhibited by gas-water systems in high permeability rock, the transition zones approach sharp interfaces. In the limiting 
case, the pressures near the caprock of the reservoir are related by the difference in fluid densities, and the vertical distances between the interface and the caprock.

Since the three-dimensional simulator described in

this dissertation is easily reducible to lower dimensions, the interest in pseudo-three dimensional features is we11 justified. The limiting vertical equilibrium (VE) concept first investigated by coates (8) is briefly described in Appendix D. This concept permits the calculation of 1imiting pseudo-capillarity and relative permeability curves. In this dissertation, these pseudo-saturation functions played a very important role in reducing the scope of sensitivity studies. A recent paper by J. Martin (73) presents a rigorous method of partial integration of multi-phase flow equations, together with a theoretical foundation of $\mathrm{VE}$ condition. 


\section{CHAPTER VI}

\section{APPLICATION OF THE NUMERICAL SIMULATOR: SENSITIVITY STUDIES OF RP AND PC \\ IN RESERVOIR MODELING}

In order to demonstrate the application of the hysteretic flow simulator, a two-dimensional grid network representation of a partially gas saturated aquifer, at initial capillary-gravity equilibrium was used. A constant reservoir thickness of 10 feet was assumed so that laboratory capillarity and relative permeability data could be employed without adjustment. Gas was injected for 120 days at an explicit rate of $1000 \mathrm{MSCF} / \mathrm{D} / \mathrm{well}$. Subsequent production lasted 690 days with the simultaneous flow of gas and water totaling 1000 MSCF of equivalent gas volume per day per well. After 810 days, production was stopped and injection was resumed at previous rates. Table I shows the saturations of the gas invaded blocks at three levels of the simulation time; 120,810 , and 1,110 days. Table II shows the corresponding dynamic pressures computed for the injection and edge blocks. Figure 10 illustrates the grid system. Figure 11 depicts the injection-production schedule for each well of the example simulation Run A1. At the simulation time level of 810 days, gas was 
TABLE I

SATURATION $\left(S_{w}\right)$ MAPS FOR THE EXAMPLE SIMULATION RUN A1

\begin{tabular}{|c|c|c|c|c|}
\hline & $I=3$ & 4 & 5 & 6 \\
\hline \multicolumn{5}{|c|}{$\mathrm{T}=120$ Days } \\
\hline $\begin{array}{r}\mathrm{J}=1 \\
2 \\
3 \\
4 \\
5 \\
6\end{array}$ & $\begin{array}{l}1.000 \\
1.000 \\
1.000 \\
1.000 \\
0.971 \\
0.961\end{array}$ & $\begin{array}{l}1.000 \\
1.000 \\
1.000 \\
0.957 \\
0.380 \\
0.288\end{array}$ & $\begin{array}{l}1.000 \\
1.000 \\
0.971 \\
0.380 \\
0.200 * \\
0.200\end{array}$ & $\begin{array}{l}1.000 \\
1.000 \\
0.962 \\
0.288 \\
0.200 \\
0.200\end{array}$ \\
\hline \multicolumn{5}{|c|}{$\mathrm{T}=810$ Days } \\
\hline $\mathrm{J}=\begin{array}{r}1 \\
2 \\
3 \\
4 \\
5 \\
6\end{array}$ & $\begin{array}{l}1.000 \\
1.000 \\
1.000 \\
1.000 \\
0.945 \\
0.930\end{array}$ & $\begin{array}{l}1.000 \\
1.000 \\
1.000 \\
0.902 \\
0.666 \\
0.613\end{array}$ & $\begin{array}{l}1.000 \\
1.000 \\
0.944 \\
0.666 \\
0.511 * \\
0.328\end{array}$ & $\begin{array}{l}1.000 \\
1.000 \\
0.931 \\
0.613 \\
0.328 \\
0.201\end{array}$ \\
\hline \multicolumn{5}{|c|}{$\mathrm{T}=1110$ Days } \\
\hline $\mathrm{J}=\begin{array}{r}1 \\
2 \\
3 \\
4 \\
5 \\
6\end{array}$ & $\begin{array}{l}1.000 \\
1.000 \\
1.000 \\
1.000 \\
0.931 \\
0.963\end{array}$ & $\begin{array}{l}1.000 \\
1.000 \\
1.000 \\
0.893 \\
0.644 \\
0.628\end{array}$ & $\begin{array}{l}1.000 \\
1.000 \\
0.931 \\
0.644 \\
0.466^{*} \\
0.323\end{array}$ & $\begin{array}{l}1.000 \\
1.000 \\
0.964 \\
0.629 \\
0.324 \\
0.204\end{array}$ \\
\hline & & ut Well & Location & \\
\hline
\end{tabular}


TABLE II

PRESSURE PROF ILES FOR EXAMPLE STMULATION RUN A1 (psi)

\begin{tabular}{|c|c|c|c|}
\hline \multicolumn{4}{|c|}{$I=5}$. \\
\hline & 120 Days & 810 Days & 1110 Days \\
\hline $\begin{array}{r}J=1 \\
2 \\
3 \\
4 \\
4 \\
5 \\
6\end{array}$ & $\begin{array}{l}975 \\
990 \\
1010 \\
1015 \\
1020^{*} \\
1022\end{array}$ & $\begin{array}{l}637 \\
615 \\
585 \\
530 \\
470 * \\
472\end{array}$ & $\begin{array}{l}1000 \\
1017 \\
1035 \\
1092 \\
1135 * \\
1105\end{array}$ \\
\hline \multicolumn{4}{|c|}{ *Input-Output Well Location } \\
\hline
\end{tabular}




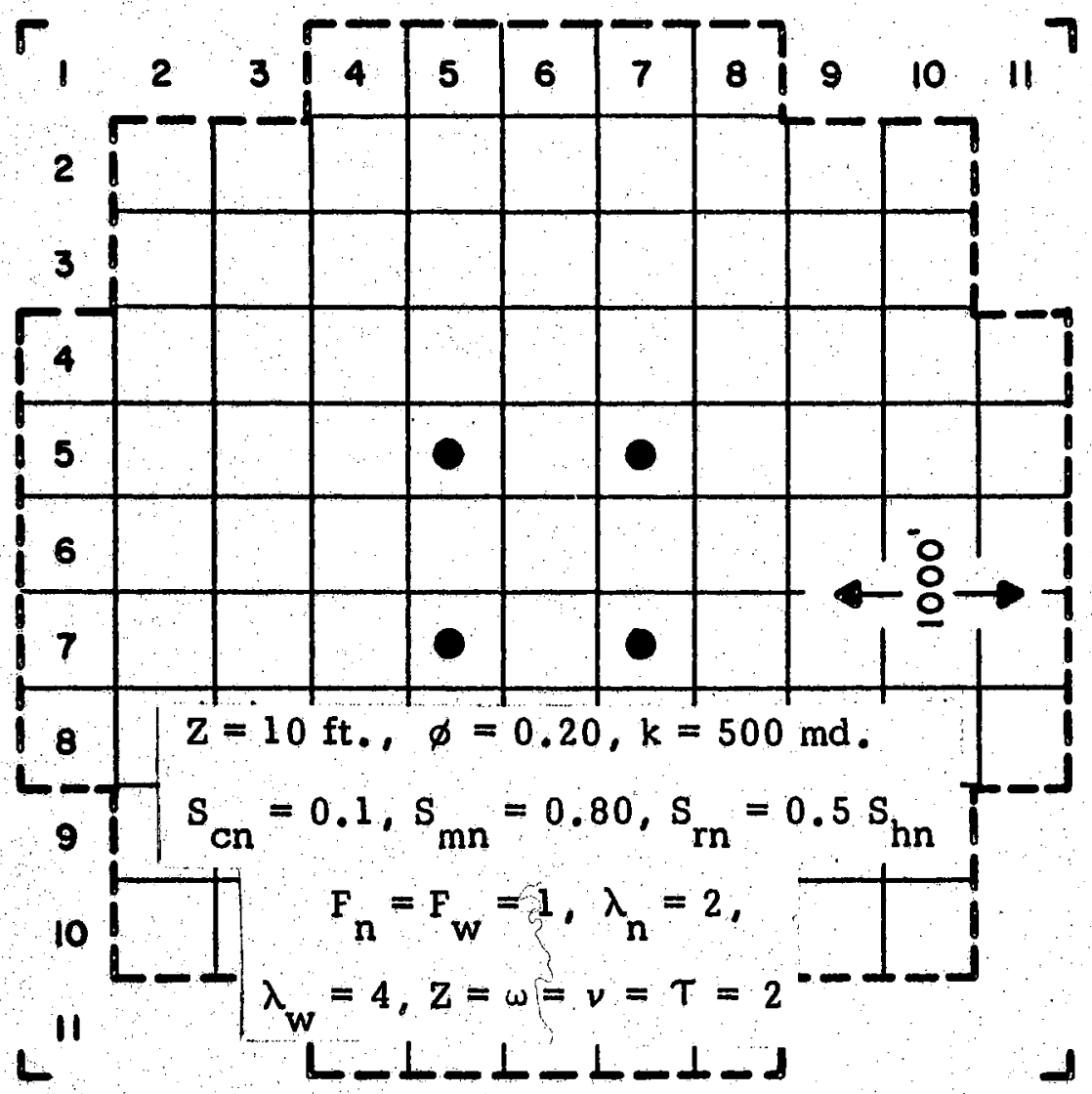

- WELL LOCATION

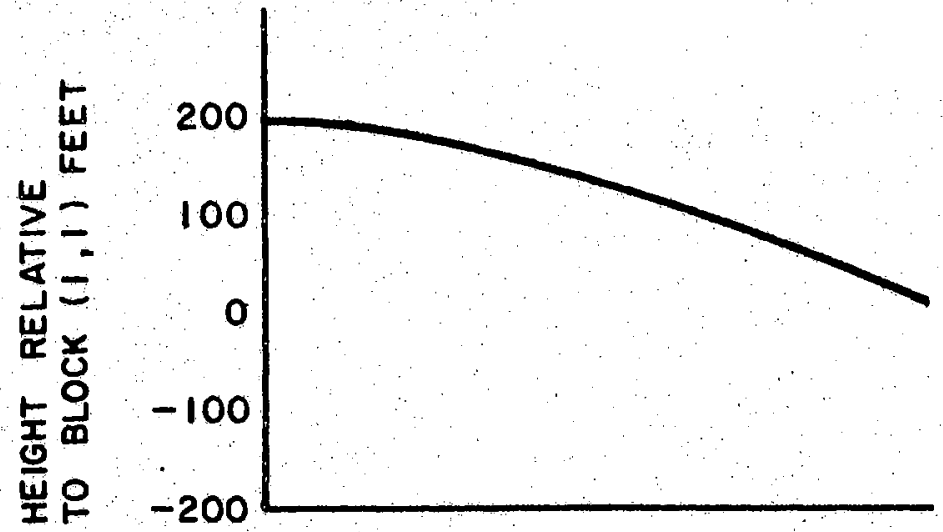

Figure 10. Two-Dimensional Grid Network Used in Simulation Run Groups A, B, C, D 


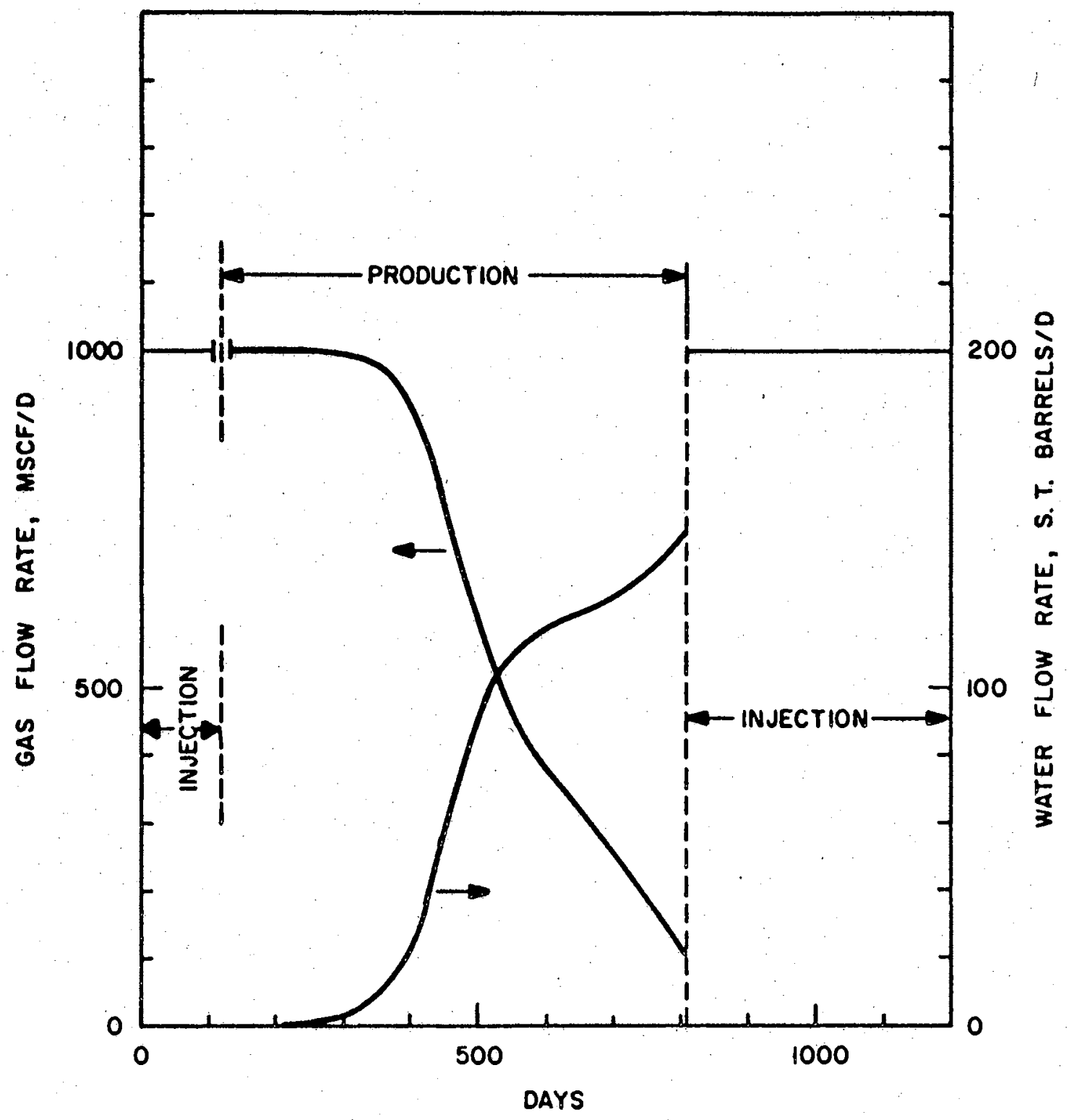

Figure 11. Injection-Production Schedule for Each Well in Simulation Run A1 
trapped in the gas invaded blocks, with RPN=O, excepting those identified by $I=5,6,7$ and $J=5,6,7$. In the blocks containing the active wells, gas saturation was near the residual level: $S_{g}=0.511$ with $R P N$ reduced to 0.015 , as compared to $\mathrm{S}_{\mathrm{rg}}=0.4$.

In reservoir studies questions arise on the methods of reconciling laboratory measured relative permeability and capillary pressure data with prototype reservoir and model conditions. The purpose of this chapter and Appendix $\mathrm{E}$ is to show, by numerical simulation of hypothetical reservoirs, how RP and PC data affect the computation of pressure and saturation distributions, and to shed light into possible methods of reconciling laboratory data and reservoir conditions.

Excepting a few special applications, the numerical simulators are constructed in a Cartesian coordinate system. The finer is the grid network encompassing a given reservoir region, the closer is the representation of the fundamental flow equations by the difference system. Ideally, grid blocks should be smaller near active wells than farther away. However, the usual range of reservoir dimensions and the number of active wells are such that the establishment of a fine grid network requires a very large number of grid points, even in two-dimensional studies, thus increasing computer memory storage requirements and processing time. Hence, the modelers are forced to use grid networks with large size blocks in order to proceed with an economically 
feasible simulation study.

Two key points must be realized before embarking on a sensitivity analysis. First, the number of variables in reservoir simulation and their ranges of possible values are such that the application of the classical sensitivity analysis methods involving most, if not all, the variables is prohibitive, and beyond the scope of this research. Hence, base permeability, porosity, fluid properties, number and location of wells, injection-production schedules, and most importantly, the grid block size and structural position were standardized to typical values throughout this work. Thus, the sensitivity of the saturation and pressure distributions to RP and PC data, considered in this paper is relative to the specified values of the "frozen" variables. Secondly, in numerical simulation studies, a transformation from the rectangular coordinates into a radial system is made to describe the flow toward (or away from) the wellbore, within a given well block, in accordance with the internal boundary conditions specified for that block. Since it is customary to assume steady-state or quasi steady-state flow conditions within a well block, after the numerical solution of the difference equations representing the flow system, at a given time, RP's directly relate the flow rates and the corresponding pressure difference between the dynamic block pressure and the well-bore pressure; that is, an $n$-fold error in a $R P$ value causes an $n$-fold error in the terminal flow rate or the difference between the dynamic and 
we11-bore pressures, whichever happens to be the dependent variable. Therefore, the accurate representation of $R P$ in well blocks is first order important. On the contrary, PC does not normally enter flow calculations within a well block. Consequently, the sensitivity studies were limited to simulation runs concerned with the influence of $R P$ and $P C$ data on block-to-block material transfer.

Two types of studies were made: The first type involved gas injection into virgin or partially gas saturated aquifers; and the second type considered gas (and water) withdrawal from aquifer-reservoir systems in initial capillary-gravity equilibrium.

Simulation studies were made with 1) curvilinear RP data characterized by $\lambda_{\mathrm{n}}=2$ and $\left.\lambda_{\mathrm{W}}=4,2\right)$ 1inear RP data where $\lambda_{n}=\lambda_{w}=1$, and 3) vertical equilibrium RP data, for a given critical gas saturation (8). It was decided that $R P$ data could always be assumed to be straight lines between the saturation extrema, in the absence of laboratory data, or in cases where simulator conditions (i.e. large grid blocks) did not permit direct use of laboratory data and criteria for reconciling laboratory data to simulator conditions were not available. Consequently, if simulator calculations were not appreciably affected when $\lambda_{n}$ was changed from 2 to 1 , and $\lambda_{w}$ from 4 to 1 , by convention of this dissertation, it was concluded that the simulation case involved was insensitive to laboratory RP data. Three types of $\mathrm{PC}$ versus $\mathrm{S}$ relationship were used: 1) curvilinear $\mathrm{PC}$, 
2) linear PC, and 3) vertical equilibrium PC.

The simulation runs for the sensitivity studies were made on three different two-dimensional grid systems, using a constant thickness of 10 feet, uniform permeability of $500 \mathrm{md}$, and uniform porosity of 20 percent. The reservoir thickness of 10 feet was selected because laboratory RP and PC data need practically no adjustment even if vertical equilibrium conditions prevailed, as shown later in the Appendix E. Two-dimensional areal (one layer) grid systems were used to reduce computation cost. Furthermore, the behavior of a three-dimensional system, with approximately 10 feet thick layers, should be the composite of individual layer performances. It will be shown that, as the thickness of a reservoir or thicknesses of the layers of a reservoir increase, PC and RP data tend towards straight lines between the adjusted saturation extrema $\left(S_{0}, S_{n}, S_{n n}\right)$. Hence, the sensitivity of the pressure and the saturation distributions to variations in laboratory RP and PC data curvature vanishes as the reservoir thickness increases.

It is beyond the scope of this dissertation to present all the simulation runs made during the sensitivity studies; typical hypothetical case studies are covered in the Appendix E to support the conclusions summarized in the next chapter. 


\section{CHAPTER VIIT}

\section{SUMMARY, CONCLUSIONS, AND RECOMMENDATIONS}

The primary objective of this work was the development of a numerical simulator for the three-dimensional flow of two immiscible, compressible fluid phases in porous media, including the effects of gravity, and hysteretic relative permeability and capillarity. In order to reach this objective, the set of non-linear partial differential equations governing this flow process was solved by numerical techniques, and a corresponding computer program was written to instruct a scientific computer to perform saturation and phase potential (or pressure) calculations, relative to a set of specified initial and boundary conditions. The successful application of the numerical simulator to specific cases of hypothetical reservoir modeling is covered in Appendix E. Since the development of the simulator for hysteretic flow in porous media was the primary objective, detailed accounts of the development process are presented in the body and appendices of this dissertation.

The secondary objective of this work was the investigation of the sensitivity of the simulator performance to rigorous representation of hysteresis in capillarity and relative permeability. Since the hysteresis phenomena in 
this data are not any more significant than the member curves defining the hysteresis loops, it was decided to simply investigate the sensitivity of the simulator to drainage or imbibition relative permeability and capillarity data, by simulating semi-hypothetical drainage and imbibition processes. Numerous conclusions and recommendations were made on the basis of simulation run evaluations in Appendix E. The following is a summary of these conclusions and recommendations:

1. Computational techniques for rigorous representation of the dynamic hysteresis in relative permeability and capillarity in the numerical simulation of two-phase, immiscible flow through porous media were introduced. The application of the hysteretic flow simulator to three-dimensional problems requires very long computer processing times, even for coarse grid systems. For example, the hysteretic model simulated the performance of a cyclic, $11 \times 11 \times 3,4$-well system for 100 simulation days, while consuming as much computer (B-5500) time as it took the semihysteretic model to simulate an equivalent $11 \times 11 \times 1$ grid system for 1,600 days. This being the case, the consideration of the hysteresis in $\left(S_{g n}\right)$ and the corresponding shift in the quasi-linear RP and PC curves appear 
to be the extent of rigor needed in simulating cyclic systems within reasonable computing times.

2. As the individual block size increases, the employment of intermediate scanning RP-PC curves become rather academic, since a large-block grid system coupled with adjusted or unadjusted laboratory RP-PC data from core samples cannot simulate the prototype behavior with accuracy warranting the use of scanning data.

3. In simulating the process of initial gas injection into a thin aquifer, the simulation results are highly sensitive to the critical gas saturation, $S_{0}$, drainage interference exponents $\lambda_{n}$ and $\lambda_{w}$, and drainage interference coefficients $F_{n}$ and $F_{w}$, in that order. As the gas saturation grows, the drainage interference coefficients, $F_{n}$ and $F_{w}$, gain importance. The critical gas saturation for the gas input block and neighboring blocks are higher than the values obtained from core samples; they should, preferably, be calculated by a fine grid radial model simulating the saturation distribution about a typical input well, within an area covered by input block and adjoining blocks of the coarse-grid simulator. 
4. In simulating the process of initial gas injection into or production from an aquifer where the conditions permit the assumption of dip-normal equilibrium, rock PC and RP data must be adjusted to reflect that equilibrium. For such systems thicker than 10 feet, the simulator sharply loses its sensitivity to all the parameters governing laboratory PC and $R P$ data, excepting the residual phase saturations.

5. In simulating the process of gas production from a thin reservoir subject to water influx, the simulation results are highly sensitive to initial saturation distribution, residual gas saturation, the imbibition interference coefficient $E_{w}$, and the saturation weighting factors used to average RP's for two adjacent blocks exhibiting high saturation contrast. Simulator sensitivity to interference exponents $\lambda_{\mathrm{n}}$ and $\lambda_{\mathrm{w}}$ is appreciably smaller in this case.

6. For systems starting with primary (or first) drainage, critical gas saturation, and hysteresis in historical high and residual gas saturations are of first order importance. The recommended method is to establish the relationship between the historical high and 
residual saturations by testing core samples in the laboratory. If this is not possible, the petroleum technology literature should be consulted for such data on similar rock-fluid systems. It is highly advisable to first use detailed grid models of reservoir regions influenced by typical wells in order to establish model critical gas saturations of regions covered by the over-all simulator, instead of using critical saturations measured in the laboratory. For cyclic gas-water systems of low capillarity, a single curve (adjusted VE or rock curve) representing the primary drainage $P C$ is sufficient, provided that block-toblock transfer of gas is cut off by zero relative permeability at the residual gas saturation. For high capillarity systems, the dynamic capillarity hysteresis envelope, without scanning curves, is sufficient.

7. The requirement for rigor in describing the RP on active well pressures and flow rates diminishes with distance from the wells, much the same way as the influence of base permeability on well performance decreases away from the active wells. For some cyclic system simulation studies, it may be sufficient to track the saturation history of well blocks and 
consider hysteresis only in these blocks.

8. If the reservoir size requires the use of large dimension blocks and/or if the geometry and properties of the reservoir are not known well enough to set-up accurate external-internal boundary conditions, accuracy in $R P$ and $P C$ data is certainly not worth the price.

9. It appears that, unless the limitations on today's scientific computing machines are drastically overcome in the near future, additional applied research efforts should be directed into the development of methods for reconciling laboratory data with model conditions to properly simulate prototype reservoir behavior, rather than obtaining more accurate laboratory data, excepting saturation extrema. In other words, numerical simulators of the type used in this study, which simulate the behavior of actual systems of large dimensions, cannot, in most cases, use the laboratory data directly without adjustment. The development of more adequate methods for resolving laboratory-modelprototype reservoir data differences is highly dependent upon the reconciliation of the prototype behavior and the model 
predictions. This calls for extensive simulation applications to reservoirs with better known geometry and properties. 


\section{BIBLIOGRAPHY}

(1) Evrenos, A. I., and E. A. Rejda. "A Digital Computer Application to the Investigation of Aquifer Properties." Jour. Pet. Technology (July, 1966), 827 .

(2) Evrenos, A. I. "Digital Computer Programs for the Derivation of the Resistance Function of a Gas Reservoir Subject to Water Drive and Prediction of the Reservoir Pressure." (Unpub. Mechen-510 project report submitted to the Mech. Engr. Dept. of Oklahoma State University, 1966.)

(3) Evrenos, A. I. "A New Approach to Matching the Pressure History and to Predict Future Pressure Behavior of Aquifer-Gas Storage Reservoir Complexes." (Consulting work report for Northern Natural Gas Company, Omaha, Nebraska, Apri1, 1967).

(4) Douglas, J. Jr., D. W. Peaceman, and H. H. Rachford, Jr. "A Method for Calculating Multi-Dimensional Immiscible Displacement." Trans. Amer. Inst. Mech. Engrs., Vol. 216 (1949), 297 .

(5) Fagin, R. G., and C. H, Stewart, Jr. "A New Approach to the Two-Dimensional, Multi-Phase Reservoir Simulator." Soc. Pet. Engr. J. (June, 1966), 175.

(6) Goodin, ,C. S. , et al. "A Numerical Study of Waterflood Performance in a Stratified System With Cross-Flow." J. Pet. Tech. (June, 1966), 765 .

(7) Nielsen, R. I. On the Flow of Two ImmiscibleIncompressible Fluids in Porous Media. Ann Arbor: University of Michigan Press, 1962 .

(8) Coates, K. H., et al. "Simulation of ThreeDimensional, Two-Phase Flow in Oil and Gas Reservoirs." Soc. Pet. Engr. J. (December, 1967), 377 .

(9) Collins, R, E. Flow of Fluids Through Porous Materials: New York: Reinhold Publishing co., 1961. 
(10) Comer, A. G. "Fluid Mechanics of Gas Storage in Aquifers." (Unpub. paper, A.G.A. meeting, Pittsburgh, Pennsylvania, May 13, 1963.)

(11) Comer, A. G., et al. "Gas Storage Project No. 3." (Unpub. final report to Northern Natural Gas Company, Omaha, Nebraska, October, 1963.)

(12) Woods, E. G., and A. G. Comer. "Saturation Distribution and Injection Pressure for a Radial Gas Storage Reservoir." Trans. Amer. Inst. Mech. Engrs., Vol. $225(196 \overline{2), 1}, 3 \overline{89 .}$

(13) Woods, E. G. "An Investigation of Cyclic, Immiscible, Two-Phase Flow in Porous Media." Stillwater: Oklahoma State University Press, May, 1963.

(14) Foster, W. S. "Relative Permeability Hysteresis." (Student paper for SPE of AIME, 1963.)

(15) Comer, A. G. "Cyclic Capillary Pressure Characteristics-Relative Permeability Characteristics of St. Peter Formation and Lower Franconia Formation." (Unpub. report to Northern Natural Gas Company, ,. Omaha, Nebraska, August, 1965.)

(16) Melrose, J. C. "Wettability as Related to Capillary Action in Porous Media." Soc. Pet. Engr. J. (September, 1965), 259.

(17) Morrow, N. R., and C. C. Harris. "Capillary Equilibrium in Porous Materials." Soc. Pet. Engr. J. (June, 1965), 15 .

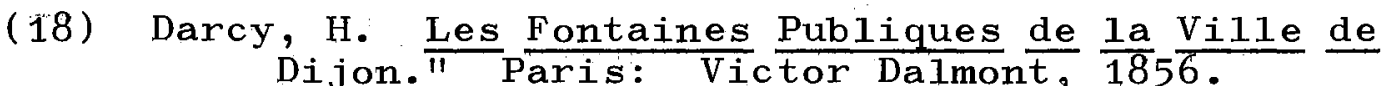

(19) Hubbert, M. D. "Darcy Law and the Field Equations of the Flow of Underground Fluids." Trans. Amer. Inst. Mech. Engrs., Vol. 207 (1956), 222 .

(20) Uren, L. C., and E. J. Bradshaw. "Experimental Study of Pressure Conditions Within the Oil Reservoir Rock in The Vicinity of a High Pressure Producing Well." Trans. Amer. Inst. Mech. Engrs., Vol. 98 (1932), 438 .

(21) Cloud, W. F. "Verification of Pressure Gradient With Distance of Rectilinear Flow of Gas Saturated Oil and Unsaturated Oi.1 Through Unconsolidated Sands." Trans. Amer. Inst. Mech. Engrs., Vo1. 86 $(1930) ; 337$. 
(22) Muskat, M., and M. W. Meres. "The Flow of Heterogeneous Fluids Through Porous Media." Physics, Vol. $7(1936), 346$.

(23) Botset, H. G. "Flow of Gas-Liquid Mixtures Through Consolidated Sand." Trans. Amer. Inst. Mech. Engrs., Vol. 136 (1940), 91 .

(24) Geffen, T. M., et al. "Experimental Investigation of Factors Affecting Laboratory Relative Permeability Measurements." Trans. Amer. Inst. Mech. Engrs., Vol. $192(19 \overline{51),} 99$.

(25) Osoba, J. S., et al. "Laboratory Measurement of Relative Permeability." Trans. Amer. Inst. Mech. Engrs., Vol. $192(195 \overline{1), 47}$

(26) Sarem, A. M. "Three Phase Relative Permeability Measurements by Unsteady-State Method." Trans. Amer. Inst. Mech. Engrs., Vol. 237 (1966), 11 .

(27) I everett, M. "Capillary Behavior in Porous Solids." Trans. Amer. Inst. Mech. Engrs., Vol. 142 (1941), 107 .

(28) Pickell, J. J., et al. "The Application of AirMercury and Oil-Air Capillary Pressure Data in the Study of Pore Structure and Fluid Distribution." Trans. Amer. Inst. Mech. Engrs., Vol. $237 \overline{(1966), \overline{107} .}$

(29) Buckley, S. F., and M. C. Leverett. "Mechanism of Fluid Displacement in Sands." Trans. Amer. Inst. Mech. Engrs., Vol. 146 (1942), 107 .

(30) Terwillinger, P. L., et al. "An Experimental and Theoretical Investigation of Gravity Drainage Performance." Trans. Amer. Inst. Mech. Engrs., Vol. $192(1952), 285$.

(31) Southwell, R. V. Relaxation Methods in Theoretical Physics. Oxford: Oxford University Press, 1946.

(32) Crank, J., and P. Nicholson. "A Practical Method for Numerical Integration of Partial Differential Equations of the Heat Conduction Type." Proc. Camb. Phil. Soc., Vol. $43(1947), 50$.

(33) Frankel, S. P. "Convergence Rates of Iterative Treatments of Partial Differential Equations." J. Math. and Physics, Vol. 29 (1951), 223. 
(34) DuFort, E. C., and S. P. Frankel. "Stability Conditions in the Numerical Treatment of Parabolic Differential Equations." Math. Table Aids to Comp., Vol. 7 (1953), 135 .

(35) Bruce, G. H., O. W. Peaceman, H. H. Rachford, and J. D. Rice. "Calculation of Unsteady State Gas Flow Through Porous Media." Trans. Amer. Inst. Mech. Engrs., Vol. 198 (1953), 79 .

(36) Peaceman, D. W., and H. H. Rachford. "The Numerical Solution of Parabolic and Elliptic Differential Equations." J. Soc. Ind. Appl. Math., Vol. 3 $($ March, 1955), $2 \overline{8}$.

(37) Douglas, J., and D. W. Peaceman. "Numerical Solution of Two-Dimensional Heat Flow Problems." AIChE Journal, Vol. 1 (Dec ember, 1955), 505.

(38) Douglas, J. "On the Relation Between Stability and Convergence in the Num. Sol. of Linear Parabolic and Hyperbolic Dif. Eq." J. Soc. I. App. Math., Vol. 4 (1955), 20 .

(39) Douglas, J. "On the Numerical Integration of . . . by Implicit Methods." J. Soc. I. App. Math., Vol. 3 (March, 1955), $4 \overline{2}$.

(40) Douglas, J., and H. H. Rachford. "On the Numerical Solution of Heat Conduction Problems in Two or Three Space Variables." Trans. Amer. Math. Soc., Vol. 82 (1956), 421 .

(41) Douglas, J. "The Application of Stability Analysis in the Num. Solution of Quasi-Linear Parabolic Diff. Eqs." Trans. Amer. Math. Soc., Vo1. 89 $(1958), 484$.

(42) Douglas, J., D. W. Peaceman, and H. H. Rachford. "A Method for Calculating Multi-Dimensional Immiscible Displacement." Trans. Amer. Inst. Mech. Engrs., Vol. 216 (1959), 297 .

(43) West, W. J., W. W. Garvin, and J. W. Sheldon. "Solution of the Equations of Unsteady-State Two-Phase Flow in Oil Reservoirs." Trans. Amer. Inst. Mech. Engrs., Vol. 201 (1954), 217.

(44) Blair, P. M., J. Douglas, and R. J. Wagner. "Calculation of Linear Water Flood Behavior Including the Effects of Capillary Pressure." Trans. Amer. Inst. Mech. Engrs., Vol. 213 (1958), 96. 
(45) McEwen, C. R. "A Numerical Solution of the Linear Displacement Equation With Capillary Pressure." Trans. Amer. Inst. Mech. Engrs., Vol. 216 (1957), 412 .

(46) Sheldon, J. W., et al. "One-Dimensional, Incompresible, Non-Capillary, Two-Phase Fluid Flow in a Porous Medium." Trans. Amer. Inst. Mech. Engrs., Vol. 216 (1959), 290.

(47) Douglas, J. "Alternating Direction Methods for Three Space Variables." Numerische Mathematik, Vol. 4 $(1962), 41$.

(48) Quon, D. , P. M. Dranchuk, and S. R. Allada. "A Stable, Explicit, Computationally Efficient Method for Solving Two-Dimensional Mathematical Models of Petroleum Reservoirs." (Unpub. paper presented at Pet. and Natural Gas Division, CIM, Calgary, Canada, May ; 1965;)

(49) Quon, D., P. M. Dranchuk, S. R. Allada, and P. K. Leung. "Application of the ADEP to TwoDimensional Natural Gas Reservoirs." Soc. Pet. Engr. J. (June, 1966), 137 .

(50) Coates, et al. "Comparison of Alternating-Direction and Successive Overrelaxation Techniques in Simulation of Two- and Three-Dimensional, Two-Phase Flow in Reservoirs." (Unpub. paper presented at 42nd Annual Fall Meeting of SPE, October, 1967.)

(51) Coates, K. H., and M. H. Terhune. "Comparison of Alternating Direction Explicit and Implicit Procedures in Two-Dimensional Flow Calculations." Soc. Pet. Engr. J. (December, 1966), 350 .

(52) Coates, K. H. "An Analysis for Simulating Reservoir Performance Under Pressure Maintenance by Gas and/ or Water Injection." Soc. Pet. Engr. J. (December, 1968), 331 .

(53) Coates, K. H. "A Treatment of the Gas Percolation Problem in Simulation of Three-Dimensional, Three-Phase Flow in Reservoirs." Soc. Pet. Engr. J. (December, 1968), 413.

(54) Irby, T, L., and W. E. Lamoreaux. "Engineering Appraisal of Mathematical Models of Petroleum Reservoirs." (Unpub. paper presented at 41st Annual Fall Meeting of SPE, October, 1966.) 
(55) Schwabe, K., and J. Brand. "Prediction of Reservoir Behavior Using Numerical Simulators." (Unpub. paper presented at 42nd Annual Fall Meeting of SPE, October, 1967.)

(56) Garrett, G. E. "Validity and Accuracy of Reservoir Simulation With a Two-Dimensional, Three-Phase, Unsteady-State Heterogeneous Matrix Model." SPE of AIME Paper No. 1881 (October, 1967).

(57) Briggs, J. E., and T. N. Dixon. "Some Practical Considerations in the Numerical Solution of TwoDimensional Reservoir Problems." SPE of AIME Paper No. 1879 (October, 1967).

(58) Hassler, G. L., et al. "The Role of Capillarity in Oil Production." Trans. AIME, Vol. 155 (1945), 155 .

(59) Crowe11, D. C., G. W. Dean, and A. G. Loomis. "Efficiency of Gas Displacement From a WaterDrive Reservoir." U. S. Dept. of Interior Bureau of Mines Report of Investigations No. 6735 $(1966)$.

(60) Holmgren, C. R., and R. A. Morse. "Effect of Free Gas Saturation in Oil Recovery by Water Flooding." Trans. Amer. Inst. Mech. Engrs., Vol. 192 (1951), $135-140$.

(61) Dyes, A. B. "Production of Water-Driven Reservoirs Below Their Bubble Point." Trans. Amer. Inst. Mech. Engrs., Vo1. $201(1954 \overline{, 240-\overline{24 .}}$

(62) Kyte, J. R., R. J. Stanclift, S. C. Stephan, Jr., and L. A. Rapoport. "Mechanism of Water Flooding in the Presence of Free Gas." Trans. Amer. Inst. Mech. Engrs., Vo1. $207\left(1956 \overline{{ }_{1}} 215-\overline{221 .}\right.$

(63) Land, C. S. "Calculation of Imbibition Relative Permeability for Two- and Three-Phase Flow From Rock Properties." Trans. Amer. Inst. Mech. Engrs., Vol. $243(1 \overline{968),} 1 \overline{49-156 .}$

(64) Naar, J., R. J. Wygal, and J. H。 Henderson. "Imbibition Relative Permeability in Unconsolidated Porous Media." Trans. Amer. Inst. Mech. Engrs., Vol. 225 (1962), 13-17.

(65) Kozeny, J. "Wiss. Wien Math. Natur Klasse (Abt. IIa)." Sitzber Akad; Vol. 136 (1957), 271. 
(66) Rose, W., and W. A. Bruce. "Evaluation of Capillary Character in Petroleum Reservoir Rock." I. Pet. Tech. (May, 1949).

(67) Rose, W. "Theoretical Generalizations Leading to the Evaluation of Relative Permeability." Trans. Amer. Inst. Mech. Engrs. (May, 1949), 111 .

(68) Marsha11, T. J. "A Relation Between Permeability and Size Distribution of Pores." J. Soil Science, Vol. 9 (1958).

(69) Wyllie, M. R. G., and G. H. F. Gardner. "The Generalized Kozeny-Karman Equation - Its Application to Problems of Multi-Phase Flow in Porous Media." World Oil (March, 1958), 121.

(70) Naar, J., and J. H. Henderson. "An Imbibition Model Its Application to Flow Behavior and the Prediction of Oil Recovery." Trans. Amer. Inst. Mech. Engrs., Vol. 222 (June, 1961), 61 .

(71) Bolt, K. W. Jr. A Study of Cyclic Capillary Pressure Effects. Stīliwater: $\overline{\text { Oklahoma State University }}$ Press, May, 1965.

(72) Carter, R. D., and G. W. Tracy. "An Improved Method for Calculating Water Influx." Trans. Amer. Inst. Mech. Engrs., Vol. 219 (1960), 58.

(73) Martin, J. C. "Partial Integration of Equations of Multi-Phase Flow." Soc. Pet. Engr. J., Vol. 8 (December, 1968), $37 \overline{0}$ 。

(74) Richtmyer, R. D. Difference Methods for InitialValue Problems. New York: Interscience Publishers, 1957 .

(75) Van Everdingen, A. F., and W. Hurst. "The Application of Laplace Transformation to Flow Problems in Reservoirs." Trans. Amer. Inst. Mech. Engrs., Vol. $186(1949) ; 305$.

(76) Hurst, W. "The Simplification of Material Balance Formulas by the Laplace Transformation." Trans. Amer. Inst. Mech. Engrs., Vol. 213 (1958), 292 .

(77) Van Poollen, H. K., E. A. Breitenbach, and D. H. Turnau. "Treatment of Individual Wells and Grids in Reservoir Modeling." Soc. Pet. Engr. J., Vol. 8 (December, 1968), 34 1. 


\title{
APPENDIX A
}

\section{EQUATIONS OF INTERMEDIATE DRAINAGE -}

IMBIBITION RP AND PC

\author{
Expressions for Intermediate Drainage Curves \\ With Branching Points on \\ Imbịbition Curves
}

$$
\begin{aligned}
& R P N \equiv U N I M+(U N D R-U N T M)\left(Z_{n} S_{5}\right)^{\nu_{n}} \\
& =F_{n}\left(1-S_{2}\right)^{\lambda_{n}}\left\{\left(Z_{n} S_{5}\right)^{\nu_{n}}+S_{3} \omega_{n}\left[1-\left(Z_{n} S_{5}\right)^{\nu_{n}}\right]\right\} \\
& R P W \equiv U W T M-(U W I M-U W D R)\left(Z_{W}^{\prime} S_{5}\right)^{\nu_{W}} \\
& =F_{W} S_{I} \lambda_{W}\left\{\left(Z_{W} S_{5}\right)^{\nu_{W}}\left[1-E_{W}+E_{W} S_{4}{ }^{\omega_{W}}\right]\right. \\
& \left.+E_{w}^{\prime}\left(1-S_{4} w_{w}\right)\right\}+E_{w} S_{4} w_{w}\left[1-\left(z_{w} S_{5}\right)^{\nu_{w}}\right]
\end{aligned}
$$

where

$$
\begin{aligned}
Z_{n}, Z_{N}= & \text { linkage coefficients for } N W F \text { and } W F \\
\nu_{n}, \nu_{N}= & \text { intermediate drainage interference } \\
& \text { exponents for } N W F \text { and } W F .
\end{aligned}
$$

When $Z_{\mathfrak{n}}=Z_{\mathfrak{w}}=1$, intermediate drainage scanning curves extend from $S_{1 \text { n }}$ to $S_{h n}$. Since $S_{h n}$ is the historical maximum SNWF, for this case, scanning curves merge into the bounding drainage curves at $S_{n}=S_{n n}$. Whenever $S_{n n}<S_{n} \leq$ $\mathrm{S}_{\mathrm{m} n}$, scanning curves do not exist. As Z's increase, 
scanning drainage curves merge into bounding drainage curves at progressively smaller values of $S_{n}$. If $Z_{n}=Z_{w}=$ $1 / S_{5}$, the intermediate scanning curves become vertical segments interconnecting the bounding imbibition and drainage curves. The form of Equation (A-1) is also suitable to express PC for this case; that is,

$$
P C \equiv P C D R+(P C D R-P C I M)\left(Z_{c} S_{5}\right)^{\nu_{0}}
$$

where $Z_{0}$ and $\nu_{0}$ have functions analogous to $Z_{n}$ and $\nu_{n}$, respective1y.

$$
\begin{aligned}
& \text { Expressions for Intermediate Drainage Curves } \\
& \text { With Branching Points on Imbibition } \\
& \text { Scanning Curves } \\
& \text { RPN } \equiv A+(\text { UNDR }-A)\left(Z_{n} S_{5}\right)^{w_{n}} \\
& \text { RPW } \equiv B-(B-U W D R)\left(Z_{w} S_{5}\right)^{w_{w}} \\
& \text { PC } \equiv C+(P C D R-C)\left(Z_{c} S_{5}\right)^{w_{w}}
\end{aligned}
$$

where

$$
\begin{aligned}
& A=\text { UNDR }-(\text { UNDR }- \text { UNIM })\left(Z_{n} S_{6}\right)^{T_{n}} \\
& B=\text { UWDR }-(\text { UWIM }- \text { UWDR })\left(Z_{w} S_{6}\right)^{T_{H}} \\
& C=\operatorname{PCDR}-(\operatorname{PCDR}-\operatorname{PCIM})\left(Z_{c} S_{6}\right)^{T_{c}}
\end{aligned}
$$

where

$\tau=$ intermediate imbibition interference exponent.

Equations (A-7 to A-9) describe the loci of RP-PC 
curves for the imbibition process preceding the current drainage process. Obviously, a drainage curve, which branches from a point on the preceding imbibition scanning curve, lies between that imbibition curve and the bounding drainage curve. Using the relationships given earlier for UNDR, ..., PCIM, Equations (A-4 to A-6) can be expanded. However, the forms given above are more efficient for numerical computation.

$$
\begin{aligned}
& \text { Expressions for Intermediate Imbibition Curves } \\
& \text { With Branching Points on } \\
& \text { Bounding Drainage Curves } \\
& \mathrm{RPN}=\mathrm{F}_{\mathrm{n}}\left(1-\mathrm{S}_{2}\right)^{\lambda_{\mathrm{n}}}\left[1-\left(\mathrm{Z}_{\mathrm{n}} \mathrm{S}_{6}\right)^{T_{\mathrm{n}}}\left(1-\mathrm{S}_{3}{ }^{\omega_{\mathrm{n}}}\right)\right] \\
& =\text { Equation }(\mathrm{A}-7) \\
& R P W=F_{W} S_{I} \lambda_{W}\left\{1-\left(Z_{W} S_{6}\right)^{T}\left[1-E_{W}\left(1-S_{4} W_{W}\right)\right]\right\} \\
& +E_{w}\left(Z_{w} S_{6}\right)^{\top} S_{4} w_{w} \\
& =\text { Equation }(\mathrm{A}-8) \\
& P C=\text { Equation }(A-9) .
\end{aligned}
$$

Expressions for Intermediate Imbibition Curves

$$
\begin{aligned}
& \text { With Branching Points on } \\
& \text { Drainage Scanning Curves }
\end{aligned}
$$

$$
R P N \equiv D-(D-U N I M)\left(Z_{n} S_{6}\right)^{\omega_{n}}
$$




$$
\begin{aligned}
R P W & \equiv E+(U W I M-E)\left(Z_{w} S_{6}\right)^{W_{w}} \\
P C & \equiv F-(F-P C I M)\left(Z_{c} S_{6}\right)^{W_{c}}
\end{aligned}
$$

where $\mathrm{D}, \mathrm{E}$, and $\mathrm{F}$ are given by Equations ( $\mathrm{A}-1, \mathrm{~A}-2$, and $\mathrm{A}-3$ ), respectively. 


\author{
APPENDIX, B \\ FLOW EQUATIONS GOVERNING THE THREE-DIMENSIONAL, \\ TWO-PHASE, COMPRESSIBLE, IMMISC IBLE, \\ HYSTERETIC FLOW IN POROUS MEDIA
}

\title{
Differential System
}

Consider an infinitesimal element of volume in a field of fluid flow through a porous medium. Let the element be of volume V; porosity $\varphi$; and saturated with at least two homogeneous, compressible, and immiscible fluids.

Let $S$ be the closed surface bounding the region $V$ of the porous medium. Suppose $x, y ; z$ to be the orthogonal cartesian axes. Define $\bar{n}$ to be the normal to the surface (positive outward); and $\alpha, \beta, \zeta$ to be the angles the normal makes with the positive $x, y, z$, axes respectively.

In the absence of sources or sinks within V, material balance requires that:

\section{MASS RATE INTO V - MASS RATE OUT OF V = MASS RATE ACCUMULATION IN V.}

Let $u_{p}$ be a vector in the field of fluid flow representing the superficial velocity of fluid $f$, continuous in the region with continuous partial derivatives. In vector form : 


$$
\begin{aligned}
& \bar{u}_{q}=u_{1} i+u_{z_{p}} j+u_{3_{q}} k \\
& \bar{n}=\cos \alpha i+\cos \beta j+\cos \zeta k .
\end{aligned}
$$

Material balance for phase $f$ can now be formulated as follows :

$$
-\iint_{S}\left(\rho_{p} \bar{u}_{i}\right) \cdot \bar{n} d S=\int f \int \frac{\partial\left(\rho_{P} S_{p} \varphi\right)}{\partial_{t}} d V .
$$

"Divergence Theorem" or "Green's Theorem in Space" states that,

$$
\iint_{S}\left(\rho_{f} \bar{u}_{p}\right) \cdot \bar{n} d S=\iint_{V} \nabla \cdot\left(\rho_{p} \bar{u}_{f}\right) d V
$$

where $\nabla$ is the vector operator (Del, Nabla) defined by:

$$
\nabla \equiv i \frac{\partial}{\partial x}+j \frac{\partial}{\partial y}+k \frac{\partial}{\partial y}
$$

From expressions (B-3) and (B-4) it follows that:

$$
-\iiint_{V} \nabla \cdot\left(\rho_{p} u_{p}\right) d V=\iiint_{V} \frac{\partial\left(\rho_{p} S_{p} \varphi\right)}{\partial_{t}} d V .
$$

Assume the porosity $\varphi$ of $V$ will not change in time. Then,

$$
\iiint \nabla \cdot\left(\rho_{p} u_{\varphi}\right)+\varphi \frac{\partial\left(\rho_{p} S_{p} \varphi\right)}{\partial_{t}} d V=0 .
$$

For this integral to be zero, the integrand must be zero at all points inside the boundary of the volume $\mathrm{V}$. 
Therefore, the negative divergence of the flow vector $u_{p}$ is the rate of accumulation of the corresponding fluid:

$$
-\nabla \cdot\left(\rho_{p} \bar{u}_{p}\right)=\varphi \frac{\partial\left(\rho_{p} u_{p}\right)}{\partial_{t}} .
$$

Equation (B-8) is the continuity (or material balance) equation for the fluid $f$ in the volume $V$.

Assume Darcy's Law, which relates the superficial

velocities to pressure (or potential) gradients, is valid for each fluid; then,

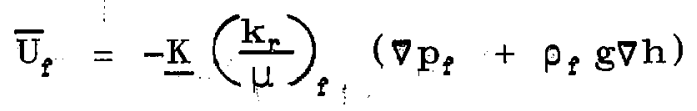

where,

$$
\underline{\mathbf{K}}=\left[\begin{array}{lll}
k_{1} & 0 & 0 \\
0 & k_{2} & 0 \\
0 & 0 & k_{3}
\end{array}\right]
$$

is the diagonal base permeability matrix obtained from the general permeability matrix (expressed in tensor form) by orienting the coordinate axes ( $x, y, z)$ orthogonal to the principal axes of the porous body of Volume $V$.

Define "Real Flow Potential" by:

$$
\Phi_{p} \equiv \int_{p_{\odot}}^{p_{\rho}} \frac{d p}{\rho_{f}(p)} \pm \frac{g h}{144 g_{c}}
$$

The real flow potential combines the pressure and gravity gradients into a single variable and also considers the variations of pressure dependent gas-law deviation factor. 
The definitions of the real flow potential and the superficial velocity lead to the following relationships:

$$
\nabla p_{\rho}+\frac{\rho_{\rho} g}{144 g_{c}} \nabla h=\rho_{p} \nabla \Phi_{f}
$$

Thus,

$$
\begin{aligned}
& \bar{u}_{p}=-\underline{K} k_{r} / \mu_{p} \rho_{p} \nabla \Phi_{p} \\
& =\left[\begin{array}{l}
u_{p_{x}} \\
u_{p} \\
u_{p}
\end{array}\right]_{f}=-\left(k_{v} \rho / \mu\right)_{p}\left[\begin{array}{ccc}
k_{x} & 0 & 0 \\
0 & k_{y} & 0 \\
0 & 0 & k_{z}
\end{array}\right]\left[\begin{array}{l}
\partial \Phi / \partial x \\
\partial \Phi / \partial y \\
\partial \Phi / \partial z
\end{array}\right]_{p}
\end{aligned}
$$

where $k_{x}^{\prime}$, $k_{y}$, and $k_{z}$ are the base permeabilities along the three principal axes of the porous medium.

Define "Formation Volume Factor" for fluid $f$ by:

$$
B_{f} \equiv \frac{\rho_{f}}{\rho_{f}} \frac{(\text { at reservoir conditions })}{(\text { at standard conditions })}
$$

Specific weights appearing in the material balance equations $(B-3, B-4, B-5, B-6, B-7, B-8)$ can now be replaced by $1 / B_{p}$ to express the material balance at standard conditions. Combination of the continuity equation (B-8) and Darcy's Law (B-12) yield for fluid $f$ :

$$
\nabla \cdot\left\{\left(\frac{\mathbf{k}_{x} \rho}{\mu B}\right)_{t}\left(\underline{K} \circ \nabla \Phi_{p}\right)\right\}=\varphi \frac{\partial}{\partial_{t}}(S / B)_{p} \cdot
$$

One should continue the mathematical formulation of the problem to include two homogeneous, compressible, and immiscible fluids in the porous body such as gas and water. 
Material balance also dictates that gas and water share the available pore space in $\mathrm{V}$; that is:

$$
S_{w}^{\prime}+S_{g}=1
$$

where $S$ is the phase saturation (fraction of pore volume occupied by one of the two fluids).

Define capillary pressure, $p_{c}$, by:

$$
\mathbf{p}_{\mathrm{G}} \equiv \mathbf{p}_{\mathbf{g}}-\mathbf{p}_{W}
$$

The displacement: of one fluid by another in the pores of a porous medium is either aided or opposed by the surface forces of the capillary pressure, which is the difference in pressure across the fluid-fluid interface caused by the interfacial tension. Two-phase capillary pressure and relative permeabilities have been successfully correlated with phase saturation. The empirical correlation functions used in this work to calculate the capillary pressure, and the phase relative permeabilities are of the form:

$$
\begin{aligned}
& \mathrm{p}_{\mathrm{e}}=\mathrm{p}_{\mathrm{g}}(\mathrm{S} *, \mathrm{C} *) \\
& \mathbf{k}_{\mathbf{r}}=\mathbf{k}_{\mathbf{r}}(\mathrm{S} *, \mathrm{C} * \underline{\mathrm{K}})
\end{aligned}
$$

where,

$$
\begin{aligned}
& S^{*}=\left\{S_{g}, \Delta S_{g}, S_{m g}, S_{h_{g}}, S_{h_{B}}, S_{I_{g}}, S_{1_{B}}, S_{g_{g}}, S_{c g}\right\} \\
& C^{*}=\text { Set of empirical coefficients and exponents } \\
& \underline{K}=\text { Diagonal base permeability matrix. }
\end{aligned}
$$


Specific empirical expressions for the explicit calculation of $P C, R P N$, and RPW are discussed in detail in the body of this text. - Since there are only two fluids saturating $V$, define:

$$
S=S_{w} ; 1-S=S_{B}
$$

From the definition of the real flow potential, it follows that,

$$
\begin{gathered}
p_{B}=\bar{\rho}_{g} \Phi_{g} \text { and } p_{w}=\bar{\rho}_{w} \Phi_{W} \\
\frac{\partial p_{B}}{\partial_{t}}=\bar{\rho}_{B} \frac{\partial \Phi_{g}}{\partial_{t}} \text { and } \frac{\partial p_{w}}{\partial_{t}}=\bar{\rho}_{W} \frac{\partial \Phi_{W}}{\partial_{t}}
\end{gathered}
$$

where, $\bar{\rho}_{g}$ and $\rho_{w}$ are mean-value specific weights over the infinitesimal element of time.

Capillary pressure can now be related to phase potentials by:

$$
p_{G}=p_{B}-p_{W}=\rho_{B} \Phi \rho-\rho_{W} \Phi_{W} \cdot
$$

Write Equation (B-15) for water and gas:

$$
\begin{gathered}
\nabla \cdot\left\{\left(\frac{k_{r} \rho}{\mu \mathrm{B}}\right)_{w}\left(\underline{K} \cdot \nabla \Phi_{w}\right)\right\}=\varphi \frac{\partial}{\partial t}\left(S / B_{w}\right) \quad(B-24) \\
\nabla \cdot\left\{\left(\frac{k_{r} \rho}{\mu B}\right)_{B}\left(\underline{K} \cdot \nabla \Phi_{w}\right)\right\}=\varphi \frac{\partial}{\partial_{t}}\left((1-S) / B_{B}\right) . \quad(B-25)
\end{gathered}
$$

Consider the right side of Equation (B-24). The operation implies, 


$$
\varphi \frac{\partial(S / B)}{\partial_{t}}=\varphi\left[\frac{1}{B_{w}} \frac{\partial S}{\partial t}+\frac{S \partial\left(1 / B_{s}\right)}{\partial_{t}}\right] .
$$

where,

$$
\begin{gathered}
\frac{\partial S}{\partial t} \equiv \frac{\partial S_{k}}{\partial_{t}}=\frac{d S}{d p_{c}} \frac{\partial p_{e}}{\partial t}= \\
S^{\prime}\left(\frac{\partial p_{g}}{\partial_{t}}-\frac{\partial p_{w}}{\partial_{t}}\right)=S^{\prime}\left[\rho_{g} \frac{\partial \Phi_{g}}{\partial_{t}}-\rho_{w} \frac{\partial \Phi_{w}}{\partial_{t}}\right]
\end{gathered}
$$

and,

$$
\frac{\partial\left(1 / B_{k}\right)}{\partial_{t}}=\frac{d\left(1 / B_{k}\right)}{d p_{w}} \frac{\partial p_{k}}{\partial t}=\left(1 / B_{w}\right)^{\prime} \rho_{w} \frac{\partial \Phi_{k}}{\partial t}
$$

where,

$$
\begin{aligned}
& S^{\prime}=\text { derivative of } S \text { with respect to } p_{o} \\
& \left(\frac{1}{B_{w}}\right)^{\prime}=\text { derivative of } \frac{1}{B} \text { with respect to } p_{w} . \\
& \text { Similarly, expand the right side of Equation (B-25): } \\
& \varphi \frac{\partial\left(1-S / B_{g}\right)}{\partial_{t}}=\varphi\left[\frac{1}{B_{g}} \frac{\partial(1-S)}{\partial_{t}}+(1-S) \frac{\partial\left(1 / B_{g}\right)}{\partial_{t}}\right]
\end{aligned}
$$

where,

$$
\begin{gathered}
\frac{\partial(1-S)}{\partial_{t}}=-\frac{\partial S}{\partial_{t}}=- \text { expression }(B-27) \\
\frac{\partial\left(1 / B_{g}\right)}{\partial_{t}}=\left(\frac{1}{B_{B}}\right)^{\prime} \rho_{g} \frac{\partial \Phi_{g}}{\partial_{t}} .
\end{gathered}
$$

Equations (B-24) and $(B-25)$ can now be written in the dependent variables $\Phi_{k}$ and $\Phi_{g}$ as follows: 


$$
\begin{aligned}
& \nabla \cdot\left\{\left(\frac{\mathbf{k}_{r} \rho}{\mu B}\right)_{w}\left(\underline{K} \cdot \nabla \Phi_{w}\right)\right\}=\varphi\left[\left(\frac{1}{B_{w}}\right) S^{\prime}\left(\rho_{g} \frac{\partial \Phi_{B}}{\partial_{t}}-\rho_{w} \frac{\partial \Phi_{w}}{\partial_{t}}\right)\right. \\
& \left.+S \rho_{w}\left(\frac{1}{B_{w}}\right) \cdot \frac{\partial \Phi_{w}}{\partial_{t}}\right] \\
& \nabla \cdot\left\{\left(\frac{\mathbf{k}_{r} \rho}{\mu B}\right)_{B}\left(\underline{K} \cdot \nabla \Phi_{B}\right)\right\}=\varphi\left[\left(\frac{-1}{B_{B}}\right) S^{\prime}\left(\rho_{B} \frac{\partial \Phi_{g}}{\partial_{t}}-\rho_{W} \frac{\partial \Phi_{B}}{\partial_{t}}\right)\right. \\
& \left.+(1-S) \rho_{g}\left(\frac{1}{B_{B}}\right)^{\prime} \frac{\partial \Phi_{B}}{\partial_{t}}\right]
\end{aligned}
$$

These equations form a simultaneous set of second-order non-linear partial differential equations in the dependent variables $\Phi_{W}$ and $\Phi_{\mathcal{E}}$.

The real flow potential $\Phi$ is a scalar and differentiable function of $x, y, z \cdot \nabla \Phi$ implies the operation;

$$
\nabla \Phi=i \frac{\partial \Phi}{\partial \mathbf{x}}+\mathbf{j} \frac{\partial \Phi}{\partial \mathbf{y}}+\mathbf{k} \frac{\partial \Phi}{\partial z}
$$

The divergence of a vector function $\bar{F}=F(x, y, z)$ implies the operation,

$$
\nabla \cdot F=i \cdot \frac{\partial \bar{F}}{\partial x}+j \cdot \frac{\partial \bar{F}}{\partial y}+k \cdot \frac{\partial \bar{F}}{\partial z}
$$

where, denotes dot product.

Remembering that the dot product of two vectors produces a scalar, the operation $\nabla \cdot\left(\frac{\mathbf{k}_{\mathbf{r}} \rho}{\mu \mathrm{B}}\right)(\underline{\mathrm{K}} \cdot \nabla \Phi)$ means 


$$
\frac{\partial\left(\underline{T} \frac{\partial \Phi}{\partial x}\right)}{\partial x}+\frac{\partial\left(\underline{T} \frac{\partial \Phi}{\partial y}\right)}{\partial y}+\frac{\partial\left(\underline{T} \frac{\partial \Phi}{\partial z}\right)}{\partial z}
$$

where $\underline{T}=\left(\frac{k_{r} \rho}{\mu B}\right) \underline{K}$.

It must be noted that in the Equations (B-30a and B-30b) all quantities represent average values over infinitesimal element of volume $v$, across an increment of time $\Delta \mathbf{x}=\mathrm{d} \mathbf{x}$ (except $\varphi$ and $K$ are assumed independent of time). For some of these variables, such as permeability, this is pure abstraction since they cease to have physical meanings for infinitesimal samples. However, to seek agreement between the integrals of these equations and the experimental observations; one does not compare the differential law to experiment for verification.

\section{Initial and Boundary Conditions}

The initial and boundary conditions are those statements which define the limits of connected regions, time, and the position and magnitude of imposed flows or potentials (pressures).

The problem considered in this text is an "initial value problem" since the differential system (B-30) has to be solved in the time region $t>0$ with given initial values of the potential (or pressure) functions and possibly their derivatives. It is also required that the phase potentials $\left(\Phi_{W}, \Phi_{B}\right)$ satisfy not only the differential equations throughout some domain of their independent variables 
$(x, y, z, t)$, but also some conditions on the boundaries of that domain. Such a set of requirements constitute a "boundary-value problem". If the potential(s) is specified on the boundary, one has a Dirichlet-type problem. A Neuman-type problem is one in which the spatial derivative normal to the boundary is specified. Since statements will be made about both the initial and the boundary conditions, our problem is an "initial and boundary value problem".

Let $\mathrm{L}_{x}, \mathrm{~L}_{y}, \mathrm{~L}_{z}$ be the spatial dimensions of the connected region. If at $t=0$, the two-phase system is in static equilibrium with no external forces imposed, a statement of the initial conditions is simply:

$$
\begin{aligned}
& 0 \leq x \leq L_{x} \\
& \Phi(x, y, z, 0)=\Phi_{0}(x, y, z) \text { for } 0 \leq y \leq L_{y} \\
& 0 \leq \mathrm{z} \leq \mathrm{L}_{\mathrm{z}}
\end{aligned}
$$

for each fluid, $\Phi_{\odot}:$ and $\Phi_{O_{w}}$ differ by the static capillary pressure expressed in potential units.

If at $t=0$, the two-phase system is not in static equilibrium, then the derivatives of $\Phi$ as well as $\Phi$ are required.

Every porous body is finite in volume; that is, an extremity exists in all directions where the permeability either vanishes (closed physical boundary) or becomes nearly infinite. If the permeability vanishes at the extremity of the permeable medium, which implies the absence of transverse flow, the component of the flow vector $\bar{u}$ in 
the direction perpendicular to the confining boundary must be zero. Since the flow vector $\bar{u}$ and the potential gradient at the boundary are related through Darcy's Law, a statement of the no-flow conditions at the confining boundary $b$ is:

$$
\frac{\partial \Phi}{\partial \mathrm{b}}=0
$$

or

$$
\nabla \Phi_{b}, t=0 \text { for completely confining boundary. }
$$

If the permeability is infinite at the boundary $b$, which implies constant terminal potential, then:

$$
\Phi_{\mathrm{b}, t}=\Phi_{0}(\mathrm{a} \text { constant })
$$

Sometimes one or more of the actual dimensions of a porous medium is so large that the potential transients created by imposed external forces never reach them within the time span specified to study the transient behavior. In this case, the condition at the external (infinite) boundary is:

$$
\Phi_{\infty, t}=\Phi_{0}(\text { initial static condition })
$$

At the interior boundaries, the conditions are usually more complex. Interior boundaries are the producing wells (sinks), the injection wells (sources), and the combination wells for cyclic systems.

Let $\psi(x, y, z)$ define the surface of the wellbore. In an injection well containing only one fluid, $f_{1}$, the 
potential of the injected fluid is continuous on $\psi$ and depends only one time; its value is that necessary to yield the specific injection rate or it is to be treated as a constant terminal potential. Since the flow of the other fluid, $f_{2}$, is zero on $\psi$, the component of the $f_{2}$ gradient normal to $\psi$ is zero. $\left(\Rightarrow\left(\nabla \Phi_{q_{2}}\right)=\perp_{\text {to } \psi}(B-38)\right.$.

At producing wells the conditions are the same as in the injection wells (excepting the change in the direction of flow) so long as one fluid enters the wellbore. When two-phase flow occurs, one of the two fluids in the wellbore can be assumed to be the carrying fluid and its potential on $\psi$ or its production rate specified. The concept of fluid distribution according to either static or dynamic capillary equilibrium deteriorates on $\psi$ or nearby, because at the wellbore (and nearby) the velocities are very high; that is, the flow regime is turbulent, and $P C$ can be taken as zero.

\section{Difference System}

Equations (B-30a and B-30b) cannot be solved for normally encountered initial and boundary conditions in reservoirs by analytical means. A numerical solution can be obtained by replacing the spatial and time derivatives by finite difference approximations, specifying appropriate initial and boundary conditions, and defining the magnitude and the number of time steps for which the solution is to be obtained. 
Divide the continuum of space and time into discreet (equal or unequal as the case may be). intervals and consider the dependent variables at the finite set of lattice points $x_{1}, y_{g}, z_{1}, t_{n}$ with the intervals chosen small enough to limit the truncation error. In transforming a differential system to a difference system, it is hoped that the resulting approximations to the time and space derivatives will yield systems of algebraic equations, which relate the known values of dependent variables at a time $t_{n}$ to unknown values of the dependent variables at a time $t_{n+1}$, thus permitting forward progress in time.

Previous work by numerous investigators $(38,39,40$, 41, 42) has shown that at least some of the second-order distance derivatives must be approximated in terms of unknown values at, time $t_{n+1}$. Consequently, one could use 3-D Douglas-Rachford Alternating-Direction-Implicit-Iterative (ADIP) procedure to develop the difference system. This selection of a differencing scheme is motivated by the desirability of unconditional stability, better accuracy, and simpler algebraic equations to solve. The reader who is not familiar with ADIP, is referred to several excellent technical papers published in the literature $(36-42,50$, 51).

Consider the replacement of $\frac{\partial \frac{S}{B}}{\partial t}$ by a forward difference scheme. What is needed is,

$$
\frac{\partial(S / B)}{\partial t} \cong\left(\frac{S_{n}+1}{B_{n+1}}-\frac{S_{n}}{B_{n}}\right) / \Delta t
$$


where $n$ and $n+1$ indicate the time levels at which the values of dependent variables are known and unknown, respectively. Since,

$$
\frac{\partial(S / B)}{\partial t}=\frac{1}{B} \frac{\partial S}{\partial t}+S \frac{\partial(1 / B)}{\partial t}
$$

it follows that:

$$
\frac{\frac{S_{n+1}}{B_{n+1}}-\frac{S_{n}}{B_{n}}}{\Delta T}=\frac{1}{B_{n+1}} \frac{\left(S_{n+1}-S_{n}\right)}{\Delta T}+S_{n} \frac{\frac{1}{B_{n+1}}-\frac{1}{B_{n}}}{\Delta t} . \quad(B-41)
$$

The first undifferenced multiplier is taken at time level $n+1$, and the second at $n$; otherwise the requirements of the left side of Equation (B-41) are not met. To see this, expand the right side of Equation (B-41) with any other combination of indexed undifferenced multipliers.

Compare Equation $(B-41)$ to the right side of the Equation (B-30); it is easily observed that:

$$
\begin{aligned}
& \frac{S_{n+1}-S_{n}}{\Delta t} \cong S^{\prime}\left[\rho_{g} \frac{\partial \Phi_{g}}{\partial t}-\rho_{w} \frac{\partial \Phi_{w}}{\partial t}\right] \\
& \left(\frac{1}{B_{n+1}}-\frac{1}{B_{n}}\right) / \Delta t \cong\left(\frac{1}{B}\right)^{\prime} \bar{p} \frac{\partial \Phi}{\partial t}
\end{aligned}
$$

which, in forward difference form becomes:

$$
\left(S_{n+1}-S_{n}\right) /\left(p_{c_{n+1}}-p_{a_{n}}\right)=S^{\prime}
$$

where, $S$ is a chord of $p_{c}$ versus $S$ curve. 


$$
\begin{aligned}
& \frac{\left(\frac{1}{B_{n+1}}-\frac{1}{B_{n}}\right)}{\Delta t}=\left(\frac{1}{B_{g}}\right), \rho_{g} \frac{\left(\Phi_{B_{n+1}}-\Phi_{g}\right)}{\Delta t} \\
& \frac{\left(\frac{1}{B_{n+1}}-\frac{1}{B_{n}}\right)_{w}}{\Delta t}=\left(\frac{1}{B_{w}}\right), \rho_{w} \frac{\left(\Phi_{w_{n}+1}-\Phi_{w_{n}}\right)}{\Delta t} .
\end{aligned}
$$

Use of Equations (B-42 - B-46) in Equations (B-30a and B-30b) gives :

\section{For gas}

$$
\begin{array}{r}
\frac{\varphi \partial\left(S_{g} / B_{g}\right)}{\partial t} \approx \frac{\left(\frac{S_{n+1}}{B_{n+1}}-\frac{S_{n}}{B_{n}}\right)}{\Delta t}=\frac{\varphi}{\Delta t}\left\{\frac { - 1 } { B _ { B _ { n } + 1 } } S ^ { \prime } \left[\rho_{g}\left(\Phi_{B_{n+1}}-\Phi_{g_{n}}\right)-\right.\right. \\
\left.\left.\rho_{w}\left(\Phi_{w_{n}+1}-\Phi_{w_{n}}\right)\right]+\left(1-S_{n}\right)\left[\left(\frac{1}{B_{g}}\right)^{\prime} \rho_{g}\left(\Phi_{g_{n}+1}-\Phi_{g_{n}}\right)\right]\right\} \\
(B-47 a)
\end{array}
$$

\section{For water}

$$
\begin{gathered}
\frac{\varphi \partial\left(S_{w} / B_{w}\right)}{\partial t} \approx \frac{\varphi}{\Delta t}\left\{\frac{1}{B_{w_{n}}+1} S^{\prime}\left[\rho_{g}\left(\Phi_{B_{n}+1}-\Phi_{B_{n}}\right)-\rho_{w}\left(\Phi_{w_{n}+1}-\Phi_{w n}\right)\right]\right. \\
\left.+S\left[\left(\frac{1}{B_{w}}\right)^{\prime} \rho_{w}\left(\Phi_{w_{n+1}}-\Phi_{n_{n}}\right)\right]\right\} .
\end{gathered}
$$

By factoring difference approximations to time derivatives, one obtains:

$$
\frac{\varphi \partial\left(S_{g} / B_{g}\right)}{\partial t} \approx \frac{\varphi}{\Delta t}\left\{\left[S^{\prime}\left(\frac{1}{B_{B_{n+1}}}\right) \rho_{w}\left(\Phi_{w_{n}+1}-\Phi_{w_{n}}\right)\right]\right.
$$




$$
\left.+\left[\left(1-S_{n}\right)\left(\frac{1}{B_{g}}\right)^{\prime} \rho_{g}-\left(\frac{1}{B_{g_{n}+1}}\right) S^{\prime} \rho_{g}\right]\left(\Phi_{g_{n}+1}-\Phi_{n}\right)\right\} \quad(B-48 a)
$$

and Equation (38) becomes:

$$
\begin{aligned}
\frac{\varphi \partial\left(S_{w} / B_{w}\right)}{\partial t} & \approx \frac{\varphi}{\Delta t}\left\{\left[S \rho_{w}\left(\frac{1}{B}\right)^{\prime}-\left(\frac{1}{B_{w}+I}\right) S^{\prime} \rho_{w}\left(\Phi_{w+1}-\Phi_{w_{n}}\right)\right.\right. \\
& \left.+\left[\left(\frac{1}{B_{w+1}}\right) S^{\prime} \rho_{B}\left(\Phi_{B+1}-\Phi_{n}\right)\right]\right\} .
\end{aligned}
$$

Multiply both sides of Equations (B-48a and B-48b) by the product of space intervals $\Delta x, \Delta y, \Delta z$ and denote:

$$
\mathrm{PV}=\varphi \Delta \mathrm{x} \cdot \Delta \mathrm{y} \cdot \Delta \mathrm{z} \cdot
$$

Define:

$$
\begin{gathered}
G 1=\frac{P V}{\Delta t}\left[S \rho_{w}\left(\frac{1}{B_{w}}\right)^{\prime}-S^{\prime}\left(\frac{1}{B_{w+1}}\right) \rho_{w}\right] \\
H 1=\frac{P V}{\Delta t}\left[S^{\prime} \rho_{B} \frac{1}{B_{w+1}}\right] \\
G 2=\frac{P V}{\Delta t}\left[S^{\prime}\left(\frac{1}{B_{B}+1}\right) \rho_{w}\right] \\
H 2=\frac{P V}{\Delta t}\left[(1-S) \rho_{B}\left(\frac{1}{B_{B}}\right)^{\prime}-S^{\prime} \rho_{g}\left(\frac{1}{B_{B n}+1}\right)\right] .
\end{gathered}
$$

Use of Equations (B-49-B-53) in Equations (B-48a and B-48b) yield:

$$
\varphi \Delta x \Delta y \Delta z \frac{\partial\left(S_{w} B_{w}\right)}{\partial t} \approx G 1 \Delta_{t} \Phi_{w}+H 1 \Delta_{t} \Phi_{B}
$$




$$
\varphi \Delta \mathbf{x} \Delta \mathrm{y} \Delta \mathrm{z} \frac{\partial\left(\mathrm{S}_{\mathrm{g}} / \mathrm{B}_{\mathrm{g}}\right)}{\partial \mathrm{t}} \approx \mathrm{G} 2 \Delta_{\mathrm{t}} \Phi_{\mathrm{w}}+\mathrm{H} 2 \Delta_{\mathrm{t}} \Phi_{\mathrm{g}}
$$

where $\Delta_{t}$ is forward time difference operator. The difference approximation of the right side of Equations (B-30a and $\mathrm{B}-30 \mathrm{~b}$ ) is now complete.

The left sides of the Equations (B-30a and $B-30 b)$ are of the form,

$$
\nabla \cdot \underline{T} \nabla \bar{\Phi}=\frac{\partial}{\partial x}\left(T_{x} \frac{\partial \Phi}{\partial x}\right)+\frac{\partial}{\partial y}\left(T_{y} \frac{\partial \Phi}{\partial y}\right)+\frac{\partial}{\partial z}\left(T_{z} \frac{\partial \Phi}{\partial z}\right) \cdot \quad(B-55)
$$

The application of central difference approximations replaces each term of Equation (B-55) with terms of the type,

$$
\begin{gathered}
\frac{\partial}{\partial x} T_{x} \frac{\partial \Phi}{\partial x}=T_{x_{1+1 / 2}} \Phi_{1+1}-\left(T_{x_{1+1 / 2}}+T_{x_{1-1 / 2}}\right) \Phi_{1}+ \\
\frac{\frac{T_{x_{1-1 / 2}} \Phi_{1-1}}{\Delta x^{2}}}{}
\end{gathered}
$$

All the coefficients $T$ and potentials $\Phi$ in Equation (B-56) are understood to apply at lattice points $(j, 1)$.

Since in a difference system the space coordinates are defined only at points $\left(x_{1}, y_{9}, z_{1}\right)$, the mass of a "difference element" is assumed to be concentrated at the lattice points and mass transfer is permitted between adjoining lattice points along chords, provided that a nonzero chord and potential gradient exists between any two points.

The coefficient of $\nabla \Phi$ in Equations (B-30a and B-30b) 
include the diagonal absolute permeability matrix; and phase specific weight, formation volume factor, viscosity, and relative permeability. Remembering that the right sides of Equations (B-48a and B-48b) and therefore (B-30a and B-30b) were multiplied by $\Delta x \Delta y \Delta z$, define phase transmissibility $T$ such that:

$$
\begin{aligned}
& \mathbf{T x}=\text { (A) } \frac{\Delta \mathbf{x} \Delta \mathbf{y} \Delta \mathbf{z}}{\Delta \mathbf{x}^{2}}=\mathbf{A} \frac{\Delta \mathbf{y} \Delta \mathbf{z}}{\Delta \mathbf{x}} ; \mathrm{Ty}=\text { (A) } \frac{\Delta \mathbf{x} \Delta \mathbf{z}}{\Delta \mathbf{y}} ; \\
& \mathbf{T z}=\text { (A) } \frac{\Delta \mathbf{x} \Delta \mathbf{y}}{\Delta \mathbf{z}} ; \mathbf{A}=\left(\frac{\mathbf{k} \mathbf{k}_{\mathbf{r}} \rho}{\mu \mathrm{B}}\right) \text { gas or water }
\end{aligned}
$$

In three dimensional space coordinates there are a total of six chords emanating from a lattice point to the adjoining lattice points (for each phase). Representing gas by $G$ and water by $W$ the chords (or transmissibilities) leading to the point $\left(x_{1}, y_{1}, z_{1}\right)$ are:

$$
\begin{aligned}
& \mathrm{TXG}_{1-1 / 2,1,1} ; \mathrm{TXG}_{1+1 / 2, j, 1} \\
& \mathrm{TYG}_{1, j-1 / 2,1} ! \mathrm{TYG}_{1, j+1 / 2,1} \\
& \mathrm{TZG}_{1,1,1-1 / 2 !} \mathrm{TZG}_{1, j, 1+1 / 2}
\end{aligned}
$$

for gas phase, and a corresponding set for water phase. The fraction ${ }^{1 / 2}$ comes from the type of central difference approximation used in replacing the first space derivative; that is,

$$
\frac{\partial U}{\partial \mathbf{x}} \cong \frac{U_{1+1 / 2}-U_{1+1 / z}}{\Delta x}
$$


The difference analog of the differential system (B-30a and $\mathrm{B}-30 \mathrm{~b})$ can now be written as follows:

$$
\begin{aligned}
& \Delta \mathrm{TG} \Delta \Phi_{\mathrm{g}}+\mathrm{Q}_{\mathrm{g}}=\mathrm{G} 2 \Delta_{\mathrm{t}} \Phi_{W}+\mathrm{H} 2 \Delta_{t} \Phi_{\mathrm{g}} \\
& \Delta \mathrm{TW} \Delta \Phi_{w}+\mathrm{Q}_{W}=\mathrm{G} 1 \Delta_{t} \Phi_{W}+\mathrm{H} 1 \Delta_{t} \Phi_{\mathrm{g}}
\end{aligned}
$$

where,

$$
\begin{aligned}
& \Delta \mathrm{TG} \Delta \Phi_{\mathrm{g}} \Rightarrow \operatorname{TXG}_{1+i / 2, j, 1}\left(\Phi_{B_{1+1, j, 1}}-\Phi_{1, j, 1}\right) \\
& -\operatorname{TXG}_{1=1 / 2, j, 1}\left(\Phi_{q, j, 1}-\Phi_{B_{1}=1, j, 1}\right) \\
& +\ldots-\ldots+\operatorname{TZG}_{1,1,1+1 / 2}\left(\Phi_{B_{1,1,1+1}}-\Phi_{B_{1,1,1}}\right) \\
& -\mathrm{TZG}_{1,1,1-1 / 2}\left(\Phi_{1,1,1}-\Phi_{1,1,1-1}\right) \\
& \mathrm{Q}_{\mathrm{g}}=\left(\rho_{\mathrm{g}} \mathrm{q}_{\mathrm{g}}\right) \Delta \mathrm{x} \Delta \mathrm{y} \Delta \mathrm{z} \text {, typically MSCF/D } \\
& Q_{w}=\left(\rho_{w} q_{w}\right) \Delta x \Delta y \Delta z \text {, typically STB/D. }
\end{aligned}
$$

Equations (B-30a and B-30b) do not contain any sink terms. $Q_{B}$ and $Q_{W}$, rates of gas and water input (or input) are added as source (sink) terms to equations (B-60a and B-60b). If they are specified explicitly, they become interior boundary conditions; however, if potentials are specified on the interior boundary, both $Q_{g}$ and $Q_{w}$ become implicit and must be computed.

The difference system can be represented in compact 
matrix form as follows:

$$
\begin{gathered}
\delta \underline{T} \delta \bar{\Phi}+\bar{Q}=\underline{B} \Delta_{t} \bar{\Phi} \\
\underline{T}=\left[\begin{array}{rr}
T G & 0 \\
0 & T W
\end{array}\right] ; \quad \bar{Q}=\left[\begin{array}{l}
Q_{B} \\
Q_{h}
\end{array}\right] ; \quad \underline{B}=\left[\begin{array}{ll}
G 2 & H 2 \\
G 1 & H 1
\end{array}\right] ; \quad \bar{\Phi}=\left[\begin{array}{l}
\Phi \\
\Phi_{w}^{s}
\end{array}\right] .
\end{gathered}
$$

If the determinant of matrix $B$ is zero, the difference system is an analog of the prototype incompressible system, which implies that the system of Equations (B-60) is elliptic. To show this, let:

$$
\frac{1}{B_{w}}=\frac{1}{B_{E}}=1 .
$$

Then,

$$
\begin{gathered}
\mathrm{G} 1=-\frac{\mathrm{PV}}{\mathrm{DT}}\left[\left(\frac{1}{\mathrm{~B}_{\mathrm{N}_{\mathrm{n}+1}}}\right) \rho_{\mathrm{w}} \mathrm{S}^{\prime}\right]=-\mathrm{G} 2 \\
\mathrm{H} 1=\frac{\mathrm{PV}}{\mathrm{DT}}\left[\left(\frac{1}{\mathrm{~B}_{\mathrm{n}+1}}\right) \mathrm{S}^{\prime} \rho_{\mathrm{g}}\right]=-\mathrm{H} 2=\frac{\mathrm{PV}}{\mathrm{DT}}\left[\left(\frac{1}{\mathrm{~B}_{\mathrm{B}+1}}\right) \mathrm{S}^{\prime} \rho_{\mathrm{B}}\right]
\end{gathered}
$$

which means $\mathrm{G} 1=-\mathrm{G} 2, \mathrm{H} 1=-\mathrm{H} 2$, and $\mathrm{B}=\mathrm{O}$ (incompressib1e, or elliptic system).

Such a system can be directly solved by a number of methods such as Gaussian elimination, iterative ADI (easier to solve and faster), SOR (successive over-relaxation).

If the determinant of the matrix $B$ is non-zero, the system of Equations (B-60) is parabolic; that is, the fluids are considered compressible. A parabolic system can be solved by non-iterative ADI or SOR. However, iterative ADI 
allows the use of larger time steps. Furthermore, noniterative $\mathrm{ADI}$ techniques have been developed for heat transfer and single phase mass flow studies, where the conductivity or transmissibility coefficients are not dependent on saturation. The dependency of the transmissibilities on the phase saturations preclude the use of large time steps in multiphase flow simulation, since these saturation changes per time step must be kept small.

\section{Solution of the Difference System}

\section{by Iterative $\mathrm{ADI}$}

Let $H_{k}$ and $k$ denote the iteration parameter and the iteration index respectively. Rewrite the system of Equations (B-64) in compact iterative ADI form:

$\Delta \operatorname{TW} \Delta \Phi_{w}(\eta)+Q_{w}-G 1 \Delta_{t} \Phi_{u}^{(\eta, n)}-H 1 \Delta_{t} \Phi_{g}(\eta, n)=H_{k} \Sigma T W \Delta \Phi_{w}(\eta, k)$

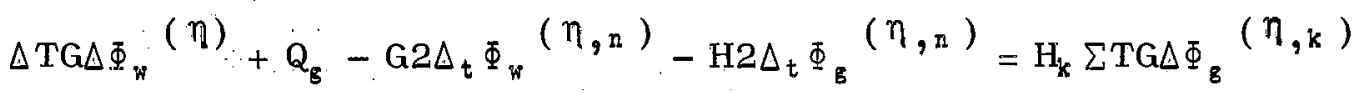

$$
\mathbf{k}, n \cong n+1
$$

where $\Sigma T G$ is the normalizing factor given by:

$$
\begin{aligned}
\sum \mathrm{TG}=\mathrm{TXG}_{1+1 / 2}, 1,1 & +\mathrm{TXG}_{1=1 / 2,1,1}+\ldots+\mathrm{TZG}_{1,1,1 \neq 1 / 2} \\
& +\mathrm{TZG}_{1,1,1-1 / 2}
\end{aligned}
$$

Iteration proceeds in three steps: 1) The X-sweep or implicit in the $\mathrm{X}$ direction, 2) The $\mathrm{Y}$-sweep or implicit in the $\mathrm{Y}$ direction, 3) The Z-sweep or implicit in the $\mathrm{Z}$ 
direction. This can be shown by expanding the system of Equations (B-65):

$$
\begin{aligned}
& \Delta_{x} \operatorname{TXW} \Delta_{x} \Phi_{W} *+\Delta_{y} \operatorname{TYW} \Delta_{y} \Phi_{W} k+\Delta_{z} \operatorname{TZW} \Delta_{z} \Phi_{W} k-G 1 \Phi_{W} *-H 1 \Phi_{g}^{*}= \\
& \dot{H}_{k} \sum T W\left(\Phi_{w}^{*}-\Phi_{w}{ }^{k}\right)-G 1 \Phi_{w}{ }^{n}-H 1 \Phi_{g}{ }^{n}-Q_{w} \\
& \Delta_{\mathrm{x}} \operatorname{TXW} \Delta_{\mathrm{x}} \Phi_{\mathrm{w}}{ }^{*}+\Delta_{\mathrm{y}} \operatorname{TYW} \Delta_{\mathrm{y}} \Phi_{\mathrm{W}} * *+\Delta_{\mathrm{z}} \operatorname{TZW} \Delta_{\mathrm{z}} \Phi_{\mathrm{w}}{ }^{\mathrm{k}}-\mathrm{G} 1 \Phi_{\mathrm{w}} * *-\mathrm{H} 1 \Phi_{\mathrm{g}} * *= \\
& \mathrm{H}_{\mathrm{k}} \sum \mathrm{TW}\left(\Phi_{\mathrm{W}}{ }^{* *}-\Phi_{\mathrm{W}}{ }^{\mathrm{k}}\right)-\mathrm{G} 1 \Phi_{\mathrm{W}}{ }^{\mathrm{n}}-\mathrm{H}_{1} \Phi_{\mathrm{g}} \mathrm{n}-\mathrm{Q}_{\mathrm{W}} \\
& \Delta_{x} \operatorname{TXW}_{x} \Phi_{w}{ }^{*}+\Delta_{y} \operatorname{TYW} \Delta_{y} \Phi_{w} * *+\Delta_{z} \operatorname{TZW}_{z} \Phi_{w}{ }^{k+1}-G 1 \Phi_{g}{ }^{k+1}-H 1 \Phi_{g}{ }^{k+1}= \\
& H_{k} \sum T W\left(\Phi_{W}{ }^{k+1}-\Phi_{W}{ }^{k}\right)-G 1 \Phi_{W} n-H 1 \Phi_{g} n-Q_{W} \cdot
\end{aligned}
$$

A corresponding system can be written for the gas phase with (w, W, G1, and H1) replaced by ( $g, G$, G2, and H2). $\Phi^{k}, \Phi^{*}, \Phi^{* *}, \Phi^{k+1}$ are successive approximations to the new time step values of $\Phi^{n+1}$. The solution of $\Phi_{w}^{*}$ from the Equation (B-66), $\Phi_{F} * *$ from the Equation $(B-67), \Phi_{*}{ }^{+1}$ from the Equation (B-68); and the solutions of $\Phi_{\mathrm{g}}{ }^{*}, \Phi_{\mathrm{g}}{ }^{*}{ }^{*}, \Phi_{\mathrm{g}}{ }^{\mathrm{k}+1}$ from the equations corresponding to the Equations (B-66 B-68) constitute (1) iteration. K iterations constitute (1) cycle; (1) cycle involves the solution of the Equations (B-66 - B-68) using parameters $\mathrm{H}_{\mathrm{k}_{1}}, \mathrm{H}_{\mathrm{k}_{2}}, \ldots, \mathrm{H}_{\mathrm{K}}$. Cycles are repeated until convergence is obtained within specified tolerances.

Consider the following increments which are differences in potentials with respect to the previous iteration: 


$$
\begin{aligned}
& \mathrm{PX} \equiv \Phi_{w} *-\Phi_{w}{ }^{k} \\
& \Phi_{w} *=\mathrm{PX}+\Phi_{w} \mathrm{~K} \\
& P Y \equiv \Phi_{W} * *-\Phi_{W} k \\
& \Phi_{\mathrm{W}} * *=\mathrm{PY}+\Phi_{\mathrm{W}}{ }^{\mathrm{k}} \\
& P Z \equiv \Phi_{W}{ }^{x+1}-\Phi_{W}{ }^{x} \\
& \Phi_{N}{ }^{k+1}=P Z+\Phi_{N}{ }^{k} \\
& \mathrm{RX} \equiv \Phi_{\mathrm{g}} *-\Phi_{\mathrm{g}} \mathrm{k} \\
& \Phi_{\mathrm{g}} *=\mathrm{RX}+\Phi_{\mathrm{g}} \mathrm{k} \\
& \mathrm{RY} \equiv \Phi_{\mathrm{g}} * *-\Phi_{\mathrm{g}} \mathrm{k} \\
& \Phi_{\mathrm{B}} * *=\mathrm{RY}+\Phi_{\mathrm{B}} \mathrm{k} \\
& R Z \equiv \Phi_{8}{ }^{k+1}-\Phi_{g} k \\
& \Phi_{\mathrm{g}}{ }^{\mathrm{k}+1}=\mathrm{RZ}+\Phi_{\mathrm{g}} \mathrm{k} \text {. }
\end{aligned}
$$

The use of these newly defined increments gives:

$$
\begin{aligned}
& \Delta_{x} \operatorname{TXW} \Delta_{x} \Phi_{W}{ }^{*}=\Delta_{x} \operatorname{TXW} \Delta_{x} P X+\Delta_{x} \operatorname{TXW} \Delta_{x} \Phi_{W}{ }^{k} \\
& \Delta_{y} \operatorname{TYW} \Delta_{y} \Phi_{W}{ }^{*}=\Delta_{y} \operatorname{TYW} \Delta_{y} P Y+\Delta_{y} \operatorname{TYW} \Delta_{y} \Phi_{W}{ }^{k} \\
& \Delta_{z} \operatorname{TZW}_{z} \Phi_{W}{ }^{k+1}=\Delta_{z} \operatorname{TZW} \Delta_{z} P Z+\Delta_{z} \operatorname{TZWTZ} \Phi_{W}
\end{aligned}
$$

also,

$$
\begin{aligned}
& \text { G1 } \Phi_{W}^{*}=\mathrm{G} 1 \mathrm{PX}+\mathrm{G} 1 \Phi_{w}{ }^{k} \\
& \text { G1 } \Phi_{W}{ }^{*}=\mathrm{G} 1 \mathrm{PY}+\mathrm{G} 1 \Phi_{W}{ }^{k} \\
& \text { GI } \Phi_{W}{ }^{k+1}=\mathrm{G} 1 \mathrm{PZ}+\mathrm{G} 1 \Phi_{W}{ }^{k} .
\end{aligned}
$$

Substituting Equations (B-70 - B-75) into Equation (B-66) yields:

$$
\begin{gathered}
\Delta_{x} \text { TXW } \Delta_{x} P X-G 1 P X-H 1 R X-H_{k} \Sigma T W P X= \\
-\Delta_{x} \text { TXW } \Delta_{x} \Phi_{W}{ }^{k}-\Delta_{y} T_{Y W} \Delta_{y} \Phi_{W}{ }^{k}-\Delta_{z} T Z W \Delta \Phi_{W}{ }^{k}
\end{gathered}
$$




$$
\begin{aligned}
& +\mathrm{G} 1 \Phi_{w}^{k}+\mathrm{H} 1 \Phi_{w}^{k}-\mathrm{G} 1 \Phi_{w}^{n}-\mathrm{H} 1 \Phi_{w}^{n}-\mathrm{Q}_{w} \\
& =\operatorname{G1}\left(\Phi_{W}{ }^{k}-\Phi_{w}^{n}\right)+H 1\left(\Phi_{g}^{k}-\Phi_{w}^{n}\right)-\Delta T W \Delta \Phi_{k}{ }^{k}-Q_{W} \\
& =-\mathrm{B} 1 \mathrm{X}=\mathrm{X} \text { direction residual. }
\end{aligned}
$$

Substitution of the Equations (B-70 - B-75) into (B-67) gives:

$$
\begin{gathered}
\Delta_{x} \text { TXW } \Delta_{x} P X+\Delta_{x} \text { TYW } \Delta_{y} P Y-G 1 P Y-H 1 R Y-H_{k} \Sigma T W P Y= \\
-G 1 \Phi_{W}^{n}-H 1 \Phi^{n}-\Delta_{x} T X W \Delta_{x} \Phi_{W}{ }^{k}-\Delta_{y} T Y W \Delta_{y} \Phi_{W}^{k}-\Delta_{z} T Z G \Delta_{z} \Phi_{W}{ }^{k} \\
+G 1 \Phi_{W}^{k}+H 1 \Phi_{k}^{k}-Q_{W}
\end{gathered}
$$

Now, solve Equation (B-76) for $\Delta_{x}$ TXW $\Delta_{x}$ PX and substitute into Equation $(B-77)$. Observing that

$$
-\Delta_{x} \operatorname{TXW}_{x} \Phi_{W}^{k}-\Delta_{y} \operatorname{TYW}_{y} \Phi_{W}^{k}-\Delta_{z} \operatorname{TZW} \Delta_{z} \Phi_{W}^{k}=-\Delta T W \Delta_{W}{ }^{k},
$$

Equation (B-77) becomes,

G1 PX $+\mathrm{H}_{1} \mathrm{RX}+\mathrm{H}_{k} \Sigma \mathrm{TW} \mathrm{PX}-\Delta \mathrm{TW} \Delta \Phi_{w}{ }^{k}-\mathrm{Q}_{w}+\mathrm{G} 1\left(\Phi_{w}{ }^{k}-\Phi_{W}{ }^{n}\right)$

$$
\begin{aligned}
& +\mathrm{H} 1\left(\Phi_{g} k-\Phi_{g}{ }^{n}\right)+\Delta_{y} \text { TYW } \Delta_{y} P Y-G 1 P Y-H 1 R Y \\
& -H_{k} \Sigma T W P Y=G 1\left(\Phi_{W}{ }^{k}-\Phi_{W}^{n}\right)+H 1\left(\Phi_{g} k-\Phi_{g} n\right) \\
& -\Delta T W \Delta \Phi_{W}{ }^{k}-Q_{W}
\end{aligned}
$$

and rearranging,

$$
\Delta_{y} \text { TYW } \Delta_{y} P Y-G 1 P Y-H 1 R Y-H_{k} \Sigma T W P Y=
$$

$-(\mathrm{G} 1+\mathrm{HK} \Sigma T W) \cdot \mathrm{PX}-\mathrm{H} 1 \mathrm{RX}=-\mathrm{B} 1 \mathrm{Y}=\mathrm{Y}$ direction residual. $(\mathrm{B}-78)$ 
Similarly, substitute Equations (B-70 - B-75) into Equation $(B-68) . \Delta_{x}$ TXW $\Delta_{x}$ PX and $\Delta_{y}$ TYW $\Delta_{y}$ PY now appear in the Equation (B-68) after this substitution. Solve Equations $(B-76)$ and $(B-78)$ for these terms, respectively, and substitute into Equation (B-68):

$$
\begin{gathered}
\Delta_{2} \mathrm{TZW} \Delta_{2} \mathrm{PZ}-\mathrm{G1} \mathrm{PZ}-\mathrm{H} 1 \mathrm{RZ}-\mathrm{H}_{\mathrm{k}} \Sigma \mathrm{TW} \mathrm{PZ}= \\
-\left(\mathrm{G1}+\mathrm{H}_{\mathrm{k}} \Sigma \mathrm{TW}\right) \mathrm{PY}-\mathrm{H} 1 \mathrm{RY}=-\mathrm{B} 1 \mathrm{Z}=\mathrm{Z} \text { direction residual. }
\end{gathered}
$$

Equations for the gas phase corresponding to the residual equations for the water phase can be developed by first writing the ADI form for the gas phase, using the increments defined by Equations (B-69b), and performing the same sequence of operations described above for the water phase. In fact, one only needs to change phase indicators ( $w, w$ ) to $(g, G)$ and variables (G1, H1, PX, PY, PZ) to (G2, H2, RX, $R Y, R Z)$, in the sequence of Equations $(B-70-B-79)$ in order to develop the following residual equations for the gas phase:

$$
\begin{aligned}
& \Delta_{\mathrm{x}} \mathrm{TXG} \Delta_{\mathrm{x}} \mathrm{RX}-\mathrm{G} 2 \mathrm{PX}-\mathrm{H} 2 \mathrm{RX}-\mathrm{H}_{\mathrm{k}} \Sigma \mathrm{TG} \mathrm{RX} \\
& =\mathrm{G} 2\left(\Phi_{w}^{k}-\Phi_{W}{ }^{n}\right)+H 2\left(\Phi_{s} k-\Phi_{w} n\right)-\Delta T G \Delta \Phi_{B} k-Q_{s}=-B 2 X \\
& \Delta_{y} T Y G \Delta_{y} R Y-G 2 P Y-H 2 R Y-H_{k} \sum T G R Y \\
& =-\mathrm{G} 2 \mathrm{PX}-\left(\mathrm{H} 2+\mathrm{H}_{\mathrm{k}} \Sigma \mathrm{TG}\right) \mathrm{RX} \quad=-\mathrm{B} 2 \mathrm{Y}
\end{aligned}
$$




$$
\begin{gathered}
\Delta_{2} \mathrm{TZG} \Delta_{2} R Z-G 2 P Z-H 2 R Z-H_{k} \Sigma T G R Y \\
=-G 2 P Y-\left(H 2+H_{k} \Sigma T G\right) R Y=-B 2 Z .
\end{gathered}
$$

\section{$\underline{X-S W E E P}$}

Let :

$$
\begin{aligned}
& \mathrm{G} 1 \mathrm{~A} \equiv \mathrm{G} 1+\mathrm{H}_{\mathrm{k}} \Sigma \mathrm{TW} \\
& \mathrm{H} 2 \mathrm{~A} \equiv \mathrm{H} 2+\mathrm{H}_{k} \Sigma T G .
\end{aligned}
$$

The fraction $1 / 2$ appearing in some of the transmissibilities in Equation (53) can be removed by defining base permeabilities as:

$$
\begin{aligned}
\mathbf{k}_{\mathbf{x}_{1}=} & \mathbf{x}-\text { direction permeability between blocks } \\
& i \text { and } i-1 \\
\mathbf{k}_{y_{1}=} & \mathbf{y}-\text { direction permeability between blocks } \\
& j \text { and } j-1 \\
k_{z_{1}=} & z-\text { direction permeability between blocks } \\
& 1 \text { and } 1-1 .
\end{aligned}
$$

Operations indicated by Equations $(B-76)$ and (B-80) yield:

$$
\begin{aligned}
& \mathrm{TXW}_{1+1} \mathrm{PX}_{1+1}-\left(\mathrm{TXW}_{1+1}+\mathrm{TXW_{1 }}+\mathrm{G} 1 \mathrm{~A}\right) \mathrm{PX}_{1} \\
&+ \mathrm{TXW}_{1} \mathrm{PX}_{1-1}-\mathrm{H} 1 \mathrm{RX}_{1}=-\mathrm{B} 1 \mathrm{X}_{1} \\
& \mathrm{TXG}_{1+1} \mathrm{RX}_{1+1}-\left(\mathrm{TXG}_{1+1}+\mathrm{TXG}_{1}+\mathrm{H} 2 \mathrm{~A}\right) \mathrm{RX}_{1} \\
&+\mathrm{TXG}_{1} \mathrm{RX}_{1-1}-\mathrm{G}_{2} \mathrm{PX}_{1}=-\mathrm{B} 2 \mathrm{X}_{1}
\end{aligned}
$$

where each TXW, TXG, and PX, RX, B1X, B2X carry subscripts 
$j$ and 1.

\section{$\underline{\text { Y-SWEEP }}$}

$\mathrm{TYW}_{\mathrm{g}+1} \mathrm{PY}_{\mathrm{g}+1}-\left(\mathrm{TYW}_{\mathrm{g}+1}+\mathrm{TYW} W_{\mathrm{g}}+\mathrm{G} 1 \mathrm{~A}\right) \mathrm{PY}_{\mathrm{g}}+\mathrm{TYW} \mathrm{PY}_{\mathrm{g}-1}-\mathrm{H} 1 \mathrm{RY_{j }}=-\mathrm{B} 1 \mathrm{Y}_{\mathrm{g}}$

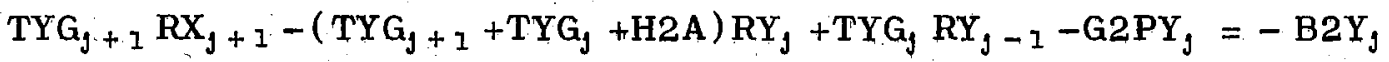

where subscripts $i$ and 1 are understood for each $T, P$, and B.

\section{Z-SWEEP}

$\mathrm{TZW}_{1+1} \mathrm{PZ}_{1+1}-\left(\mathrm{TZW}_{1+1}+\mathrm{TZW} \mathrm{W}_{1}+\mathrm{G} 1 \mathrm{~A}\right) \mathrm{PZ}_{1}+\mathrm{TZW}_{1} \mathrm{PZ}_{1-1}-\mathrm{H}_{1} \mathrm{RZ}_{1}=-\mathrm{B} 1 \mathrm{Z}_{1}$

$\mathrm{TZG}_{1+1} \mathrm{RZ}_{1+1}-\left(\mathrm{TZG}_{1+1}+\mathrm{TZG}_{1}+\mathrm{H} 2 \mathrm{~A}\right) \mathrm{RZ}_{1}+\mathrm{TZG}_{1} \mathrm{RZ}_{1-1}-\mathrm{G} \mathrm{PZ}_{1}=-\mathrm{B} 2 \mathrm{Z}_{1}$

\section{Solution of Sweep Equations}

Let :

$$
\mathbf{P}=\mathbf{P X} ; \mathbf{R}=\mathbf{R X} ; \beta=\mathrm{G} 1 \mathrm{~A} ; \quad \mathrm{b}=-\mathrm{B} 1 \mathrm{X} ; \alpha=\mathrm{H} 1
$$

Consider a Dirichlet-type problem in $\mathbf{x}$ direction, with the values of pressure specified on the boundary:

$$
P(0, n)=A ; P(N+1, n)=B
$$

where $n$ is the time index, and linear sweep system is divided into $N+1$ equal increments, and $x=\Sigma \Delta x_{1}$. Writing the equation (B-85) with $T, P$, and $R$ at $i=1,2, \ldots, N-$. 
one obtains:

$$
\begin{array}{cc}
-\left(T_{2}+T_{1}+\beta_{1}\right) P_{1}+T_{2} P_{2} & -\alpha_{1} R_{1}=b_{1}-T_{1} P_{0}=b_{1}-T_{1} A \\
T_{2} P_{1}-\left(T_{3}+T_{2}+\beta_{2}\right) P_{2} & -\alpha_{2} R_{2}=b_{2} \\
\cdot & \cdot \\
T_{N} P_{N-1}-\left(T_{N+1}+T_{N}+\beta_{N}\right) P_{N}-\alpha_{N} R_{N}=b_{N}-T_{N}+1 B
\end{array}
$$

This system may be written in compact matrix form as follows :

$$
\underline{\mathrm{T}} \overline{\mathrm{P}}+\underline{\alpha} \overline{\mathrm{R}}=\overline{\mathrm{C}}
$$

where,

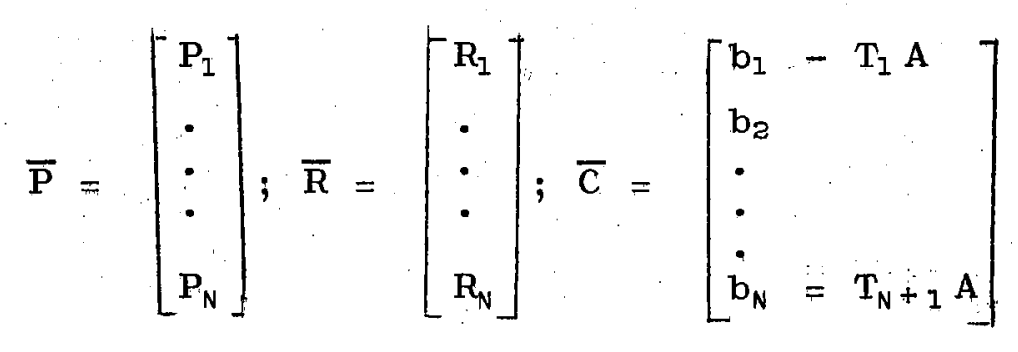

$\underline{\alpha}$ is the diagonal matrix:

$$
\underline{\alpha}=\left[\begin{array}{lllll}
\alpha_{1} & & & \\
& \cdot & & & \\
& & \cdot & & \\
& & & \alpha_{N}
\end{array}\right]
$$

and $T$ is the tri-diagonal matrix: 


\begin{tabular}{|c|c|c|c|c|c|}
\hline$-\left(T_{1}+T_{2}+\right.$ & $\mathrm{T}_{2}$ & 0 & 0 & $\cdots$ & 0 \\
\hline $\mathrm{T}_{2}$ & $-\left(T_{2}+T_{3}+\beta_{3}\right)$ & $T_{3}$ & 0 & $\cdots$ & 0 \\
\hline 0 & $\mathrm{~T}_{3}$ & $-\left(T_{3}+T_{4}+\beta_{4}\right)$ & $\mathrm{T}_{4}$ & $\ldots$ & 0 \\
\hline - & • & - & & $\ldots$ & - \\
\hline - & • & • & & $\ldots$ & - \\
\hline . & • & $\cdot$ & & $\cdots$ & - \\
\hline 0 & 0 & 0 & & & \\
\hline
\end{tabular}

By letting $P, R, \beta, b$, and $\alpha$ represent variables corresponding to those indicated by the set of transformations (B-91) for any other sweep direction equations (B-86 B-90), one can observe that they can be written in the form of the Equation (B-92). For the gas phase equations, the positions of the vectors $P$ and $R$ are interchanged. Thus, each sweep requires the simultaneous solution of the following bi-tri-diagonal system:

$$
\begin{aligned}
& \underline{T_{w}} \overline{\mathrm{P}}+\alpha_{w} \overline{\mathrm{R}}=\overline{\mathrm{C}}_{w} \\
& \underline{T_{g}} \overline{\mathrm{R}}+\alpha_{g} \overline{\mathrm{P}}=\overline{\mathrm{C}}_{8}
\end{aligned}
$$

where $w$ and $g$ are the usual phase subscripts.

Solution of the Equations (B-93a and B-93b)

Equation (B-93) may be solved by extending an algorithm developed by Richtmeyer (74) to solve the system

$$
\text { I } \overline{\mathrm{P}}=\overline{\mathrm{C}}
$$

by Gaussian elimination. Assume a property $\psi_{1}$, which is a 
relationship between $P_{1=1}$ and $P_{1}$. Similarly, assume a property $\xi_{1}$ which is a relationship between $R_{1=1}$ and $R_{1}$. Now, show that if $\psi_{1}$ and $\xi_{1}$ are "true", then by the system of Equations (3), $\psi_{1+1}$ and $\xi_{1+1}$ are also "true". If it can now be shown that $\psi_{2}$ and $\xi_{2}$ are "true", then by induction $\psi_{3} \rightarrow \psi_{n+1}$ and $\xi_{3} \rightarrow \xi_{n+1}$ are all true.

The relations assumed are:

$$
\begin{aligned}
& \mathrm{PX}_{1-1}=\mathrm{C} 1_{1} \mathrm{PX}_{1}+\mathrm{D} 1_{1} \mathrm{RX}_{1}+\mathrm{E} 1_{1} \\
& \mathrm{RX}_{1-1}=\mathrm{C} 2_{1} \mathrm{PX}_{1}+\mathrm{D} 2 \mathrm{RX}_{1}+\mathrm{E} 2_{1}
\end{aligned}
$$

Substitute $\mathrm{PX}_{1-1}$ and $\mathrm{RX}_{1-1}$ from these equations into Equations $(\mathrm{B}-85)$ and $(\mathrm{B}-86)$ :

$$
\begin{aligned}
& \mathrm{TXW}_{1+1} \mathrm{PX}_{1+1}-\left[\mathrm{TXW}_{1+1}+\left(1-\mathrm{C}_{1}\right) \mathrm{TXW}_{1}+\mathrm{G} 1 \mathrm{~A}\right] \mathrm{PX}_{1}- \\
& \left(\begin{array}{ll}
H 1-\mathrm{TXW}_{1} & \mathrm{D} 1_{1}
\end{array}\right) \mathrm{RX}_{1}=-\mathrm{B} 1 \mathrm{X}_{1}-\mathrm{TXW} \mathrm{E} 1_{1} \\
& \mathrm{TXG}_{1+1} \mathrm{RX}_{1+1}-\left[\mathrm{TXG}_{1+1}+\left(1-\mathrm{D} 2_{1}\right) \mathrm{TXW}_{1}+\mathrm{H}_{2 \mathrm{~A}}\right] \mathrm{RX}_{1}- \\
& \left(\mathrm{G} 2-\mathrm{TXG}_{1} \mathrm{C2} 2_{1}\right) \mathrm{PX}_{1}=-\mathrm{B} 2 \mathrm{X}_{1}-\mathrm{TXG}_{1} \mathrm{E} 2_{1} \cdot \\
& \mathrm{a}_{1} \mathrm{PX}_{1+1}-\mathrm{b}_{1} \mathrm{PX}_{1}-\mathrm{c}_{1} \mathrm{RX}_{1}=-\mathrm{d}_{1} \\
& \mathrm{a}_{2} \mathrm{RX}_{1+1}-\mathrm{c}_{2} \mathrm{RX}_{1}-\mathrm{b}_{2} \mathrm{PX}_{1}=-\mathrm{d}_{2}
\end{aligned}
$$

and rearranging:

$$
\begin{aligned}
& \mathrm{b}_{1} \mathrm{PX}_{1}+\mathrm{c}_{1} \mathrm{RX}_{1}=\mathrm{a}_{1} \mathrm{PX}_{1+1}+\mathrm{d}_{1} \\
& \mathrm{~b}_{2} \mathrm{PX}_{1}+\mathrm{c}_{2} \mathrm{RX}_{1}=\mathrm{a}_{2} \mathrm{RX}_{1+1}+\mathrm{d}_{2} .
\end{aligned}
$$


Simultaneously solving for $\mathrm{PX}_{1}$ and $\mathrm{RX}_{1}$,

$$
\operatorname{PX}_{1}=\frac{\left|\begin{array}{lll}
\mathrm{a}_{1} \mathrm{PX}_{1+1}+\mathrm{d}_{1} & \mathrm{c}_{1} \\
\mathrm{a}_{2} \mathrm{RX}_{1+1}+\mathrm{d}_{2} & \mathrm{c}_{2}
\end{array}\right|}{\mathrm{M}} ; \operatorname{RX}_{1}=\frac{\left|\begin{array}{lll}
\mathrm{b}_{1} & \mathrm{a}_{1} \mathrm{PX}_{1+1}+\mathrm{d}_{1} \\
\mathrm{~b}_{2} & \mathrm{a}_{2} \mathrm{RX}_{1+1}+\mathrm{d}_{2}
\end{array}\right|}{\mathrm{M}}
$$

$(\mathrm{B}-103)$

where,

$$
M=\left|\begin{array}{ll}
b_{1} & c_{1} \\
b_{2} & c_{2}
\end{array}\right|
$$

Performing the indicated operations yields:

$$
\mathrm{PX}_{1}=\left(\frac{\mathrm{a}_{1} \mathrm{c}_{3}}{\mathrm{M}}\right) \mathrm{PX}_{1+1}-\left(\frac{\mathrm{a}_{2} \mathrm{c}_{2}}{\mathrm{M}}\right) \mathrm{RX}_{1+1}+\frac{\mathrm{d}_{1} \mathrm{c}_{2}-\mathrm{d}_{2} \mathrm{c}_{1}}{\mathrm{M}}
$$

$$
R X_{1}=\left(\frac{a_{2} b_{1}}{M}\right) R X_{1+1}-\left(\frac{a_{1} b_{2}}{M}\right) P X_{1+1}+\frac{b_{1} d_{2}-b_{2} d_{1}}{M}
$$

The set of equations $(B-104)$ and $(B-105)$ are of identical form to the set of equations (B-95) and (B-96). It was, thus, shown that if the relationships (B-95) and (B-96) are "true" at $i$, they are also "true" at $i+1$. The coefficients can be obtained recursively as follows:

$C_{1+1}=\frac{a_{1} c_{2}}{M} ; D 1_{1+1}=\frac{-a_{2} c_{1}}{M} ; C 2_{1+1}=\frac{-a_{1} b_{2}}{M} ; D 2_{1+1}=\frac{a_{2} b_{1}}{M} ;$

$$
E 1_{1+1}=\frac{d_{1} c_{2}-d_{2} c_{1}}{M} ; E 2_{1+1}=b_{1} d_{2}-b_{2} d_{1} \quad(B-106)
$$

where, 


$$
\begin{aligned}
& a_{1}=\operatorname{TXW}_{1+1} ; a_{2}=T X G_{1+1} \\
& \mathrm{~b}_{1}=\mathrm{TXW}_{1+1}+\left(1-\mathrm{C}_{1}\right) \mathrm{TXW}_{1}+\mathrm{G} 1 \mathrm{~A} ; \mathrm{b}_{2}=\mathrm{G} 2-\mathrm{TXG}_{1} \mathrm{C} 2 \\
& c_{1}=H 1-\mathrm{TXW}_{1} \quad \mathrm{D} 1_{1} ; \mathrm{c}_{2}=\mathrm{TXG}_{1+\mathrm{I}}+\left(1-\mathrm{D} 2_{1}\right) \mathrm{TXG}_{1}+\mathrm{H} 2 \mathrm{~A} \\
& \mathrm{~d}_{1}=-\mathrm{B} 1 \mathrm{X}_{1}-\mathrm{TXW}_{1} \mathrm{E} 1_{1} ; \mathrm{d}_{3}=-\mathrm{B} 2 \mathrm{X}_{1}-\mathrm{TXG}_{1} \mathrm{E}_{1} \cdot \quad(\mathrm{B}-107)
\end{aligned}
$$

The starting values of the recursion coefficients at $i=2$ are obtained from Equations (B-85) and (B-86) written at $i=1$ (boundary). The set of equations (B-106) and (B-107) permit the calculation of the recursion coefficients for $i=2,3, \ldots, N+1$. The upper boundary conditions give:

$$
\begin{aligned}
& \mathrm{PX}_{\mathrm{N}+\mathrm{I}}=\mathrm{BW} \\
& \mathrm{RX}_{\mathrm{N}+\mathrm{I}}=\mathrm{BG} .
\end{aligned}
$$

Thus, from Equations (B-95) and (B-96), one obtains:

$$
\begin{aligned}
& P X_{N}=C 1_{N+1} \quad B W+D 1_{N+1} B G+E 1_{N+1} \\
& R X_{N}=C 2_{N+1} \quad B W+D 2_{N+1} B G+E 2_{N+1}
\end{aligned}
$$

and, $\left(\mathrm{PX}_{\mathrm{N}-1}, \mathrm{RX}_{\mathrm{N}-1}\right),\left(\mathrm{PX}_{\mathrm{N}-2}, \mathrm{RX}_{\mathrm{N}-2}\right), \ldots,\left(\mathrm{PX}_{1}, \mathrm{RX}_{1}\right)$ are back-calculated, in order, from Equations (B-95) and (B-96). What is meant by a "residual" and how it is used is perhaps clearer to the reader at this juncture. For instance, $B 1 X$ and $B 2 X$ are residuals of $X-$ Sweep equations $(\mathrm{B}-76)$ and $(\mathrm{B}-80)$, at any iteration level $\mathrm{k}$; they are indicative of the amount of error in the current approximations to the correct solution. The observation of the recursion 
coefficients, (B-106), show that they are directly used in the $k+1$ level iteration for the determination of the new $P X$ and $\mathrm{RX}$ values. $\mathrm{Y}$-Sweep and $\mathrm{Z}$-Sweep residuals have analogous functions at the same iteration levels $(k$ to $k+1)$.

\section{Iteration Logic}

Now it remains to develop some schemes for ascertaining when the numerical solution at any time level is sufficiently close to the "correct" solution of the analogous differential system; that is, when to stop iterating for the time level $t_{n+1}$ solution. Furthermore, iteration parameters and methods of updating pressure and saturation dependent properties are needed.

\section{Closure Criteria}

If the numerical solution at a given time step were perfect, the incremental material balance should be zero. In other words, the sum of all changes in the system over a time step should be equal to material added to or removed from the system. Due to truncation error introduced by finite difference approximation, and round-off error, incremental material balance cannot be perfect. For closure criteria, any combination of the following can be used.

a) Incremental material balance less than a specified tolerance: 
GAS BALANCE:

$$
\Sigma\left\{G 2\left(\Phi_{n+1}^{k+1}-\Phi_{n}\right)+H 2\left(\Phi_{n+1}^{k+1}-\Phi_{n}\right)\right\}=\Sigma Q_{1}
$$

\section{WATER BALANCE:}

$$
\Sigma\left\{G 1\left(\Phi_{n+1}^{x+1}-\Phi_{n}\right)+H 1\left(\Phi_{n+1}^{x+1}-\Phi_{n}\right)\right\}=\Sigma Q_{n}
$$

where $\Phi_{n+1}^{x+1}$ implies $k+1$ iteration 1 evel approximation to $\Phi_{n+1}$. Definition of incremental material balance error as a percent (or fraction) of $\Sigma Q_{\text {a }}$ for gas phase and $\Sigma Q_{w}$ for water phase yields:

\section{For water:}

$$
\left|\frac{\sum\left\{G 2\left(\Phi_{n+1}^{k+1}-\Phi_{n}\right)+H 2\left(\Phi_{n+1}^{k+1}-\Phi_{n}\right)\right\}-\sum Q_{n}}{\sum Q_{k}}\right| \leq \epsilon_{k}
$$

For gas:

$$
\left|\frac{\sum\left\{G 1\left(\Phi_{n+1}^{k+1}-\Phi_{n}\right)+H 1\left(\Phi_{n+1}^{k+1}-\Phi_{n}\right)\right\}-\sum Q_{n}}{\sum Q_{n}}\right| \leq \epsilon_{n}
$$

Typical values for $\epsilon_{*}$ and $\epsilon_{\text {, }}$ can be taken in the neighborhood of 0.001 . All values are understood to apply at the spatial position $i, j, 1$, and the summation is taken over the full ranges of $i, j, 1$. 
b) Maximum saturation change in a grid block, less than a specified tolerance:

$$
\left|S_{1,1,1, n+1}^{k+1}-S_{1,1,1, n+1}^{k}\right| \leq \epsilon_{\text {sat. }}(i . e:: 0.001)
$$

c) The sum of each of the absolute residuals over the grid system less than a specified tolerance:

$$
\begin{aligned}
& \left.\Sigma\left|\mathrm{B} 1 \mathrm{X}_{1, j, 1, \mathrm{n}+1}^{\mathrm{k}}\right| \leq \epsilon_{\mathrm{B} 1 \mathrm{X}}, \quad \text { (i.e.: } 0.05\right) \\
& \Sigma\left|\mathrm{B} 2 \mathrm{X}_{1, j, 1, \mathrm{n}+1}^{\mathrm{k}, 1}\right| \leq \epsilon_{\mathrm{B} 2 \mathrm{X}}, \quad(\text { i.e.: } 0.05) .
\end{aligned}
$$

In this dissertation, expressions (B-114), (B-115), and (B-116) were used as closure criteria.

In addition to the iteration closure criteria, cumulative material balance for each phase was used to check the "correctness" of the solution, as it marches through the time domain, and to indicate the cumulative truncation and round-off error. This was necessary because a finite (1-3) number of cycles with a finite (4-10) number of iterations were employed in an attempt to force $\Phi_{n+1}^{k+1}$ values to the desired $\Phi_{n+1}$. If the full range of specified cycles were exhausted, while the applicable closure criteria were not met, the simulation run was either stopped or the last phase potential iterates were used as potentials at the new time level $t_{n+1}$, with the hope that at $t_{n+2}$, the closure criteria would be satisfied. Thus, the cumulative material balances were checked at the end of each time step to make 
sure that the solution did not diverge as time progressed.

d) Cumulative material balance error less than a specified tolerance:

The amounts of gas and water in the system at any time $t_{n}$ are given by:

$$
\begin{aligned}
\operatorname{GIP}_{n} & =\sum_{1=1}^{N Z} \sum_{j=1}^{N Y} \sum_{1=1}^{N X} P V_{1, j, 1, n} \frac{\left(1-S_{1,1,1, n}\right)}{B_{1, j, 1, n}} \\
W_{1} P_{n} & =\sum_{1=1}^{N Z} \sum_{n=1}^{N Y} \sum_{1=1}^{N X} P V_{1, j, 1, n} \frac{S_{1, j, 1, n}}{B_{k}, j, 1, n}
\end{aligned}
$$

where $N X, N Y$, and $N Z$ are upper bounds on $i, j, l$ and $P V=$ $(\Delta x \Delta y \Delta z) \varphi \cdot$ GIP and WIP denote gas-in-place and water-inplace, respectively.

By convention of this dissertation, injection volumes are considered positive and withdrawals negative. The differences between the gross cumulative injections and withdrawals, of the two fluids, or the net volumes are given by:

$$
\begin{aligned}
& \operatorname{CUMG}_{n}=\operatorname{CUMG}_{n-1}+\sum Q_{B} \Delta t \\
& \operatorname{CUMW}_{n}=\operatorname{CUMW}_{n-1}+\sum Q_{n} \Delta t
\end{aligned}
$$

where the summation is taken over the spatial positions $i, j, l$ containing sinks (or wells). The cumulative material balance requires that:

$$
\begin{aligned}
& G I P_{0}-G_{n}=C U M P_{n} \\
& W I P_{\odot}-W_{n} P_{n}=C U M W_{n}
\end{aligned}
$$


Hence,

$$
\begin{aligned}
& \left|\frac{G I P_{0}-G I P_{n}}{C U M G_{n}}\right| \leq \epsilon_{C U M G},(i . e .: 0.1) \\
& \left|\frac{W I P_{0}-W I P_{n}}{C U M W_{n}}\right| \leq \epsilon_{C U M W},(i . e .: 0.1)
\end{aligned}
$$

were used to decide to continue with the simulation run or to terminate it.

Updating of Potential (Pressure)

Dependent Properties

After each iteration at a time step, phase potentials change; therefore, potential dependent properties must be updated. To satisfy the updating needs, tables of corresponding phase potentials, phase pressures, and phase formation volume factors were generated for each simulation case. Table look-up and interpolation techniques were used to update potential dependent properties for each potential iterate; that is, for each block $i, j, 1$.

$$
\begin{aligned}
& \left\{\Phi_{w}, \Phi_{g}\right\}_{n+1}^{k+1} \stackrel{\text { TABLES }}{\longrightarrow}\left\{P_{w}, P_{g}, P_{e}, \frac{1}{B_{w}}, \frac{1}{B_{g}}\right\}_{n+1}^{k+1} \\
& \left\{P_{0}\right\}_{n+1}^{k+1} \stackrel{\text { FROM CAPILLARITY }}{\text { RELATIONS }} \longrightarrow\{S\}_{n+1}^{k+1}
\end{aligned}
$$




$$
S^{\prime} \mathrm{k}+2=W\left(\frac{S_{n+1}^{k+1}-S_{n}}{P_{0}^{k+1}-P_{0}}\right)+(1-W) S^{\prime k+1}
$$

where $S^{\prime} k+2$ is the value of $S^{\prime}$ used over the next iteration. W dampens $S^{\prime k+2}$, since $P_{a+1}^{k+1}$ employed in calculating $S^{\prime} k+2$ is derived from phase potential iterates of the previous iteration. W was applied as a function of the iteration number :

$$
W \equiv \frac{\mathbf{k}}{\mathbf{k}_{\max }} ; 0 \leq \mathrm{W} \leq 1
$$

In two phase flow exhibiting significant hysteresis in capillarity, the calculation represented by expression (44) became a painstaking problem; because, for this type of flow, capillary pressure is not a single valued function of phase saturation. The calculation of $S_{n+1}^{k+1}$ corresponding to $\mathrm{P}_{\mathrm{a}+1}^{\mathrm{k}+1}$ itenate requires a special algorithm to determine whether $\left(\mathrm{P}_{0_{n+1}+1}^{\mathrm{k}+1}-\mathrm{P}_{0_{n}}\right)$ implies tendency to imbibe or to drain along a bounding PC curve or an intermediate scanning PC curve. Similarly, an algorithm is needed to ascertain what is implied by the value of $\left(S_{n+1}-S_{n}\right)$ for a given block. Algorithms named HYSTRACK and SEMHYSTRACK were developed to keep track of the saturation history of specified blocks, and select the appropriate subset from the set of equations given in Chapter IV for RP and PC. These algorithms are described in Appendix $C$. 


\section{Updating of Saturation Dependent Properties}

When the applicable closure criteria is met for a given iteration over the time step from $t_{n}$ to $t_{n+1}$, one obtains:

$$
\begin{aligned}
& \Phi_{n+1}^{k+1} \cong \Phi_{n+1} ; \Phi_{n+1}^{k+1} \cong \Phi_{n+1} ; S_{n+1}^{\prime k+2}=S_{n+1}^{\prime} \\
& S_{n+1}=S_{n}+S_{n+1}^{\prime}\left[\bar{\rho}_{n}\left(\Phi_{n+1}-\Phi_{n}\right)-\bar{\rho}_{n}^{\prime}\left(\Phi_{n+1}^{\prime}-\Phi_{n n}\right)\right] \\
& =S_{n}+S_{n+1}^{\prime} P_{o_{n+1}}
\end{aligned}
$$

Using $S_{n}$ and $S_{n+1}$, saturation dependent variables, $R P$ and PC, can be updated for use over the time interval $t_{n+1}$ to $t_{n+2}$. For non-hysteretic flow or when the conditions permit the replacement of hysteresis envelopes with single representative curves, both phase RP's and PC's become single valued functions of phase saturation. In this case, the updating work is straightforward. For hysteretic flow, the algorithms described in Appendix $C$ were used to update saturation dependent properties.

$$
\begin{gathered}
\text { Effect of Heterogeneity on Relative } \\
\text { Permeability-Capillarity }
\end{gathered}
$$

All hydrocarbon reservoirs are more or less heterogeneous. Absolute permeability of the typical reservoir rock varies from point-to-point. Hence, RP also varies from point-to-point. In multi-phase flow parallel to bedding planes, total resistance to flow is dominated by component 
strata of high effective RP, whereas the converse is true in flow perpendicular to bedding planes.

Semi-empirical relations for $R P$ presented in Chapter IV contain constant residual WF saturation $\left(S_{F}{ }_{w}^{\prime}=1-S_{B}\right)$. In simulating heterogeneous reservoirs, $S_{\text {m }}$ can either be treated as a subscripted variable and stored in computer memory for each grid block (or layer as the case may be), or as a function of base permeability if there is laboratory data to support the functional relation. The higher the WF residual saturation, the lower the relative permeability to NWF appears to be, at this residual saturation. This observation can be quantified in RP expressions by transforming interference coefficient $F_{B}$ from a simple multiplier to a function of residual WF saturation; i.e.

$$
F_{B}=F_{B}\left(1-S_{F_{B}}\right)=F_{B}\left(S_{z B}\right) .
$$

If enough samples of heterogeneous reservoir rock with different RP characteristics are available, Equation (B-137) can be written as a polynomial fit to the laboratory data. Such a correlation can also be used in accounting for differences in sample capillarity data.

$$
\text { In this dissertation, Leverett's (27) capillarity }
$$
retention relation was used to calculate the bounding drainage PC values for layers or grid blocks whose Klinkenberg permeability and porosity differed from those of the base block. Leverett developed the semi-empirical relation: 


$$
J\left(S_{w}\right)=\frac{P_{c}}{\sigma \cos \theta}\left(\frac{k_{a} \dot{s}}{\varphi}\right)^{\frac{1}{2}}=\frac{213.875(p s i)}{\text { dynes/cm }}\left(\frac{m d}{\text { fraction }}\right)^{\frac{1}{2}}
$$

$(B-134)$

which he accepted because it was supported by dimensional analysis and experimental data. $J\left(S_{w}\right)$ is a dimensionless function of the physical properties of rock-fluid system. The factor $(\mathrm{k} / \varphi)^{\frac{1}{2}}$ is also encounteréd in Poiseuille and Darcy models of flow in porous media, and represents the "average pore radius", thus imparting theoretical justification in Equation (B-134).

Once the capillary retention trend characterizing a given type of rock is obtained, $P C$ can be related to $J\left(S_{w}^{5}\right)$ which, in turn, is related to $S_{w}^{\prime}$ values. All that need be known are estimated values for $k_{a s}, \varphi$, and $\sigma$. In this dissertation, Equations (8) and (13) represented the PC of the base layer (or region) of the prototype reservoir. For any other layer or region, exhibiting different properties, a capillarity multiplier, $M_{\odot}$, was used. Since,

$$
\left.\left.J\left(S_{w}\right)=\frac{P_{c}}{\sigma \cos \theta}\left(\frac{k}{\varphi}\right)^{\frac{1}{2}}\right)_{\text {base }}=\frac{P_{c}}{\sigma \cos \theta}\left(\frac{k}{\varphi}\right)^{\frac{1}{2}}\right)_{\text {region } R}, \quad(B-135)
$$

it follows that:

$$
\begin{aligned}
& P_{c_{R}}=P_{o_{b}} \frac{(\sigma \cos \theta)_{R}}{(\sigma \cos \theta)_{\text {base }}} \frac{(k / \varphi)_{b \text { ase }}^{\frac{1}{2}}}{(k / \varphi)_{R}^{\frac{1}{2}}} \\
& =M_{c} P_{c} \text { base } ; M_{c}=\text { capillarity multiplier } \text {. }
\end{aligned}
$$




\section{Boundary Conditions for the \\ Numerical Simulator}

The initial and boundary conditions are statements which define the limits of the connected regions, the time, and the position and magnitude of the imposed flows. A detailed discussion of the initial and boundary conditions for the differential system was given earlier in this appendix. In this section the initial and boundary conditions actually used in the numerical simulator are described.

External Boundary Conditions

Since every porous body is finite in volume, an extremity exists in all directions where the permeability either vanishes (closed boundary) or becomes nearly infinite (constant terminal potential condition). In simulating twophase systems, it is not necessary to extend the grid network to cover all the boundaries of the prototype system, provided that their effects are properly accounted for. When the external faces of any number of grid blocks coincide with a closed prototype boundary, the transmissibilities TG and TW of these blocks normal to the boundary are set equal to zero. Otherwise, steady-state or unsteadystate WF influx-efflux methods should be applied. The simplest method of treating an open boundary, used in this work was the "aquifer strength method", whereby the WF movement across the external faces of the edge grid blocks, over a time interval, was calculated from: 


$$
q_{w b}=q_{0} \quad B_{w} \bar{\rho}_{w}\left(\Phi_{w}^{n+1}-\Phi_{w}^{n}\right)
$$

where:

$$
\begin{aligned}
q_{w_{b}}= & \text { WF movement into (or out of) the edge block } \\
& i, j, 1 \text { (typically in STB/D). } \\
q_{0}= & \text { Aquifer strength beyond the external face } \\
& \text { of block } i, j, 1 \text { (Res. Barrels/D). } \\
b \quad & \text { Subscript designating the boundary. }
\end{aligned}
$$

For simulation cases requiring more accurate representation of the effects of WF movement, outside the area covered by the grid, on the potential and saturation distributions within the grid, Carter-Tracy (72) Unsteady-State Method was utilized. This method circumvents the need for superposition calculations normally required under unsteadystate and varying rate conditions by a clever approximation of Hurst-Van Everdingen solutions (75, 76). Carter-Tracy approximate water influx equation (72) is:

$W_{n}=W_{(n=1)}+\left(t_{D_{n}}-t_{D_{(n=1)}}\right)\left\{\frac{B \Delta P_{n}-W_{\theta}(n-1) P_{D}^{\prime}\left(t_{D_{n}}\right)}{P_{D}\left(t_{D_{n}}\right)-t_{D_{(n-1}} P_{D}^{\prime}\left(t_{D_{n}}\right)}\right\}$

where:

$$
\begin{aligned}
t_{D_{n}} & =\frac{0.00633 k_{w} t_{n}}{\varphi \mu_{w} C_{t} r_{b}} \text {, dimensionless time. } \\
B & =1.1191 \varphi C_{t} h r_{b} 2 F
\end{aligned}
$$




$$
\begin{aligned}
& P_{D}^{\prime}\left(t_{D}\right)=\frac{d}{d t_{D}}\left[P_{D}\left(t_{D}\right)\right] \\
& P_{D}\left(t_{D_{n}}\right)=\text { Van Everdingen-Hurst dimensionless pressure } \\
& \text { drop. } \\
& r_{b}=\text { Radius of an imaginary circle encompassing } \\
& \text { the prototype reservoir area represented by } \\
& \text { the grid system, feet. } \\
& F=\text { Correction for limited portion of reservoir } \\
& \text { perimeter open to water influx-efflux, } \\
& \text { fraction. } \\
& c_{t}=\text { Total compressibility for the aquifer, psi-1. } \\
& W_{n}=\text { Cumulative water influx at } t_{n} \text {. } \\
& \Delta P_{n}=P_{0}-P_{n}
\end{aligned}
$$

Equation (B-138) was transformed into a function of real gas potential on the open boundary as follows. Let:

$$
\begin{aligned}
& D=P_{D}\left(t_{D_{n}}\right)-t_{D_{(n-1)}} P^{\prime}{ }_{D}\left(t_{D_{n}}\right) \\
& \Delta P_{n} \equiv P_{D}-P_{n-1}+P_{n-1}-P_{n} .
\end{aligned}
$$

Since Equation (B-138) has the dimensions of reservoir volume of water movement over a cumulative time period, the term in the brackets becomes:

\{\}$=\frac{B\left(P_{0}-P_{n-I}\right)-W_{\theta}(n-I) P_{D}^{\prime}\left(t_{D_{n}}\right)}{D}-\frac{B \rho_{w}^{-}\left(\Phi_{w n}-\Phi_{(n-I)}\right)}{D} \cdot$

Transposing $W_{(n-1)}$ to the left side of Equation (B-138) and 
dividing both sides by $t_{n}-t_{(n-I)}$ yields the mean influx rate over the $\mathrm{n}^{\text {th }}$ time interval:

$$
q_{b}, t_{n}=A_{b}+B_{b} \Delta_{t} \Phi_{w}
$$

where:

$\mathrm{b}=$ represents the boundary block

$$
\begin{aligned}
A_{b} & =\frac{\left[B\left(P_{\Theta}-P_{n-1}\right)-W_{(n-1)} P_{D}^{\prime}\left(t_{D_{n}}\right)\right] t_{D_{n}} / t_{n}}{D}(B-143) \\
& \therefore \quad \bar{p}_{w} t_{D_{n} / t_{n}}
\end{aligned}
$$

\section{Internal Boundary Conditions}

Interior boundaries are the producing wells (sinks), the injection wells (sources), and the combination wells for cyclic systems. Since the difference system used in this work is not a "purely implicit" system, there is a choice between implicit or explicit internal boundary conditions. Furthermore, at least two distinct types of boundary conditions can be set up at each well over a time interval: constant terminal rate and constant terminal pressure. The equations used in this simulator to describe twophase flow toward (production) or away from (injection) a well's bore-hole, within a single block, will be described first. Various manipulations of these equations yield boundary conditions at each well of interest. 


\section{Flow Within a Grid Block}

When reservoirs are represented by regular or irregular grid networks, the dimensions of grid blocks are many times greater than the well-bore diameters, and the calculated pressure and saturation for lattice point $i, j, 1$ are average properties of the block represented by that point. If a well is shut-in, the well pressure and the block pressure are comparable. However, if the well is active, the well pressure differes markedly from the average block pressure, also known as dynamic pressure.

If the grid system is set up so that there is at most one well per block, Darcy flow may be assumed within the well blocks. Pressures in a square or almost square finite system can be assumed to behave similar to those in a finite radial system ( 77$)$.

Since ADI solution of the difference system gives the block average pressure and saturation over any time interval, each block with an active well is treated as a finite system for flow within the block.

As before, consider the NWF to be gas and the WF to be water. Darcy's Law for radial gas flow in standard conditions is:

$$
q_{s}=\frac{(A) 2 \pi r h k p T_{s}}{\mu Z T_{R} p_{s}} \quad \frac{d p}{d r}
$$

where subscripts $s$ and $R$ refer to standard and reservoir conditions, respectively, and $A$ is the proper unit 
conversion constant.

Defining the "real gas potential" as

$$
\Phi=\int_{0}^{p} \frac{p}{Z(P)} d p
$$

and substituting Equation (B-146) into Equation (B-145) yields:

$$
q_{s}=-\frac{(A) 2 \pi r h k p T_{s}}{\mu T_{R} p_{s}} \frac{d \Phi}{d r} .
$$

Hence,

$$
\int_{r_{b}}^{r} \frac{q_{g} \mu T_{R} p_{s}}{(A) 2 \pi h k T_{s}} \frac{d r}{r}=-\int_{r_{b}}^{r} d \Phi
$$

where $r_{b}$ is the wellbore radius.

Letting,

$$
\hat{Q}=\frac{q_{s} \mu T_{R} p_{s}}{(A) 2 \pi h k T_{s}}
$$

substituting Equation (B-149) into Equation (B-148) and integrating both sides gives:

$$
Q \ln \left(\frac{r}{r_{b}}\right)=\Phi_{b}-\Phi(r) \text {. }
$$

Consider a $\Phi$ which is the integrated average potential bounded by the volume characterized some external radius, $r_{0}:$

$$
\Phi \equiv \frac{\int_{r_{b}}^{r_{a}} \Phi(r) 2 \pi r d r}{\pi r_{e}^{2}} .
$$


Solving Equation (B-150) for $\Phi(r)$ and substituting into Equation (B-151), one obtains:

$$
\begin{aligned}
\Phi & =\frac{2}{r_{b}^{2}} \int_{r_{b}}^{r_{0}}\left(\Phi_{b}-\hat{Q} \ln \frac{r_{a}}{r_{b}}\right) r d r \\
& =\Phi_{b}-\hat{Q}\left(\ln \frac{r_{b}}{r_{b}}-1 / 2\right)
\end{aligned}
$$

where,

$$
\begin{aligned}
\Phi_{b} & =\text { Potential at the well-bore. } \\
h & =\text { Block thickness in feet. } \\
\mathbf{k} & =\text { Block base permeability. } \\
T_{B} & =\text { Standard temperature (i.e. } 520^{\circ} \text { R) } \\
p_{B} & =\text { Standard pressure (i.e. } 14.73 \text { psia). }
\end{aligned}
$$

The selection of $r_{0}$, in the definition of $\Phi$ by the Equation (B-151), depends upon the detail in the reservoir definition. If few, large dimension blocks are used, it is normally assumed that $\Phi_{0}=\Phi$, and $r_{0}$ is computed from:

$$
r_{e}=\left\{\begin{array}{l}
\Delta x / 2, \text { for } \Delta x=\Delta y \\
\frac{\sqrt{x} \frac{y}{\pi},}{n} \text { for } \Delta x \neq \Delta y .
\end{array}\right.
$$

For detailed computations with numerous blocks, $r_{\bullet}$ may still be obtained from the relationship (B-153) provided that $\Phi$ in Equation $(B-152)$ is replaced by $\Phi_{\text {. calculated from }}$ the pressures of blocks adjoining the well blocks. A simple method to calculate $\Phi_{\text {, is: }}$ 


$$
\Phi_{1}=\Phi_{1}+F_{1}\left[\begin{array}{c}
\frac{4}{j=1} \\
\sum_{j=1}^{4}(h k / \mu)\left(\Phi_{j}-\Phi\right)
\end{array}\right]
$$

where $\Phi ' s$ are the block average potentials and $F$ is a weighing factor. As $F$ approaches zero, $\Phi_{\text {. approaches }} \Phi$.

Equation (B-152) is a single phase flow equation. For two-phase flow, the mobilities of both phases must be considered. Letting:

$$
\begin{aligned}
& \mathbf{M}_{s}=\left(\mathbf{k}_{\mathrm{r}} / \mu\right)_{\mathrm{B}}=\text { gas phase mobility } \\
& \mathbf{M}_{\mathrm{w}}=\left(\mathbf{k}_{\mathrm{r}} / \mu\right)_{\mathrm{w}}=\text { water phase mobility }
\end{aligned}
$$

and expressing reservoir flow rates in reservoir barrels per day gives:

$$
\begin{aligned}
& q_{g}=\frac{0.00708 M_{g} B_{B} \hat{\rho}_{b}\left(\hat{\Phi}-\Phi_{b}\right)}{\ln \frac{r_{b}}{r_{b}}-1 / 2}, \operatorname{MSCF/D} \\
& q_{B_{w}}=\frac{0.00708 M_{w} B_{w}^{\prime} h \hat{\rho}_{w}\left(\Phi-\Phi_{b}\right)}{\ln \frac{r_{b}}{r_{b}}-1 / 2}, \text { STB } / D
\end{aligned}
$$

where,

$B_{i}^{\prime}=$ gas formation volume factor, MCSF/RB.

$B_{W}=$ water formation volume factor, STB/RB.

$\hat{\rho}_{c}=$ specific weight of gas at reservoir conditions $\left(\mathrm{T}_{\mathrm{R}}\right.$ and $\left.\Phi\right)$, psi/foot.

$\hat{\rho}_{w}=$ specific weight of water at reservoir conditions. 
Constant Terminal Rate Condition (Explicit)

In this case, flow rates are explicitly specified for injection wells, and used directly as $q^{\prime}$ and $q_{w}$ in the difference system (B-65a \& b) and in X-direction residuals (Equations $\mathrm{B}-76$ and $\mathrm{B}-80$ ). The corresponding wellbore injection potentials $\Phi_{b}, \Phi_{b}$, are calculated from Equations $(B-156)$ and $(B-157)$. For production wells, gas (or water as the case may be) flow rate is specified. If the other phase is also mobile within the block, it can be simply calculated from $(B-156)$ and $(B-157)$ since the specified rate of the first phase yields $\left(\Phi-\Phi_{b}\right)$.

In 2-D cross-sectional and 3-D simulation studies, a well may be active in more than one block; hence, the total gas or water rate must be allocated to each participating block.

A simple method of allocation consists of multiplying the total injection rate into a well by phase mobility fraction; that is,

$$
q_{3}=q_{s} M_{1} / \sum_{l=1}^{L} M_{1}
$$

where $M$ is $M_{8}$ or $M_{w}^{\prime}$, and $L$ is the total number of blocks supplied by the well.

In case of production, the block flow rates are calculated from:

$$
\left.q_{8}\right)_{1}=q_{8}\left(M_{8}\right)_{1} / \sum_{1=1}^{L}\left(M_{8}\right)_{1}
$$




$$
\left.q_{F}\right|_{1}=\left.q_{g}\right|_{1} ^{\frac{\left(M_{w}\right)_{1}}{\left(M_{g}\right)_{1}}} \frac{B_{B}}{B_{w}}
$$

where $\mathrm{M}_{\mathrm{g}}, \mathrm{M}_{w}, \mathrm{~B}_{\mathrm{g}}$, and $\mathrm{B}_{w}$ are understood to apply at spatial position $i, j, 1$.

\section{Constant Terminal Pressure Condition}

In this case, $\Phi_{b}$ is specified and the flow rates are calculated from Equations $(B-156)$ and (B-157). This scheme is explicit: the known value of $\Phi$ at $t_{n}$ is used to compute the flow rates over the time interval $t_{n}$ to $t_{n+i}$. It is quite extraordinary to inject both gas and water into a well; hence, the phase being injected must be specified in addition to $\$$. For multi-block wells, allocation schemes present no special problem. Since $\Phi$ is known for each block and $\Phi_{b}$ specified, Equations (B-156) and (B-157) directly yield the block flow rates.

\section{Constant Terminal Rate Condition (Implicit)}

Implicit techniques approximate the wellbore conditions more realistically. To express the sink-source terms in the difference system (B-65a and B-65b) implicitly, let:

$$
\begin{aligned}
& q_{B}=q_{B}=C_{B}\left(\Phi_{n+1}^{k+1}-\Phi_{B}\right) \\
& q_{w}=q_{B}=C_{w}\left(\Phi_{n+1}^{k+1}-\Phi_{w b}\right)
\end{aligned}
$$

where, 


$$
\begin{aligned}
& c_{B}=0.00708 M_{B} B_{k} h \hat{\rho}_{B} /\left(\ln \frac{r_{b}}{r_{b}}-\frac{1}{2}\right) \\
& c_{w}=0.00708 M_{w} B_{w} h \hat{\rho}_{w} /\left(\ln \frac{r_{b}}{r_{b}}-\frac{1}{2}\right) .
\end{aligned}
$$

In this case of a gas well, $\Phi_{b}$ is assumed and iterations performed until the flow rate calculated from the Equation $(B-161)$ closes on the desired rate. On each iteration $\Phi_{b}$ is corrected by the relationship,

$$
\Phi_{b}^{k}=\Phi_{b}-\frac{q_{s}\left(\ln \frac{r_{b}}{r_{u}}-\frac{1}{2}\right)}{0.00708 M_{g} B_{B} h_{b}}
$$

where $g_{b}$ is the desired rate for this boundary condition. Equation (B-163) also applies to a water well when subscript g is replaced by subscript w. If the well is producing, the flow rate of water moving simultaneously with gas into the well-bore is calculated from the relationship (B-160).

For multi-block wells, the method remains essentially the same, with all subscripted terms involved in summations over the number of completion blocks for allocation purposes. In other words, $\Phi_{b}$ is first assumed and subsequently iterated on, until the sum of the flow rates, $q_{s}$, of individual blocks (1) closes on the total desired rate. Equation $(B-160)$ is used to calculate the water flow rate in each block drained by the well, after closure on total gas rate for the well. 
Constant Terminal Pressure Condition (Implicit)

In this case $\Phi_{b}$ (or $\Phi_{b y}$ as the case may be) is specified as the known internal boundary condition in any given block excited by an active well, and the relationships (B-161 and B-162) are directly used in the difference system to replace explicit $q_{B}$ and $q_{k}$.

\section{The Role of Relative Permeability in Establishing}

\section{Internal Boundary Conditions}

The computation of the we11-bore flow pressure corresponding to an explicit terminal flow rate condition, or the calculation of terminal flow rate(s) for a given well-bore pressure involves relative permeability terms in phase mobilities $M_{B}, M_{w}$. After closure of a time level $t_{n}$, the simulator computes block average saturation; hence, in simulation runs with fairly large grid blocks, significant saturation gradients may exist within a block. Furthermore, a well may be only partially completed in a given block, across a portion of the block whose saturation differs from the block average saturation. In these cases, relative permeability versus phase saturation correlations based upon laboratory data must be transformed to the prototype reservoir or simulator conditions. Various schemes have been proposed for such transformations. Those used in this dissertation will be discussed briefly in Appendix D.

Once the fluids saturating the prototype reservoir are specified, phase mobilities are controlled by phase relative 
permeabilities. Assuming steady-state or semi-steady-state flow within a block, the maximum mobility to the injected fluid is equal to or less than the mobility of the displaced fluid. This presents a difficult problem in simulating gas injection into a virgin aquifer. Possible solution methods are presented in Appendix E. 


\section{APPENDIX C}

\section{HYSTRACK ALGORITHM}

When the applicable closure criteria is met for a given iteration over a time step from $t_{m}$ to $t_{m+1}$, the simulator yields $S(i, j, 1)$. Using $S_{m}$ and $S_{m+1}$ values for each block, saturation dependent variables can be easily updated for non-hysteretic flow. However, for hysteretic flow the saturation history and the current locations of RP and PC points on $R P-S$ and $P C-S$ maps generated by the semiempirical equations must be considered. Defining a set of state indicators [I*]:

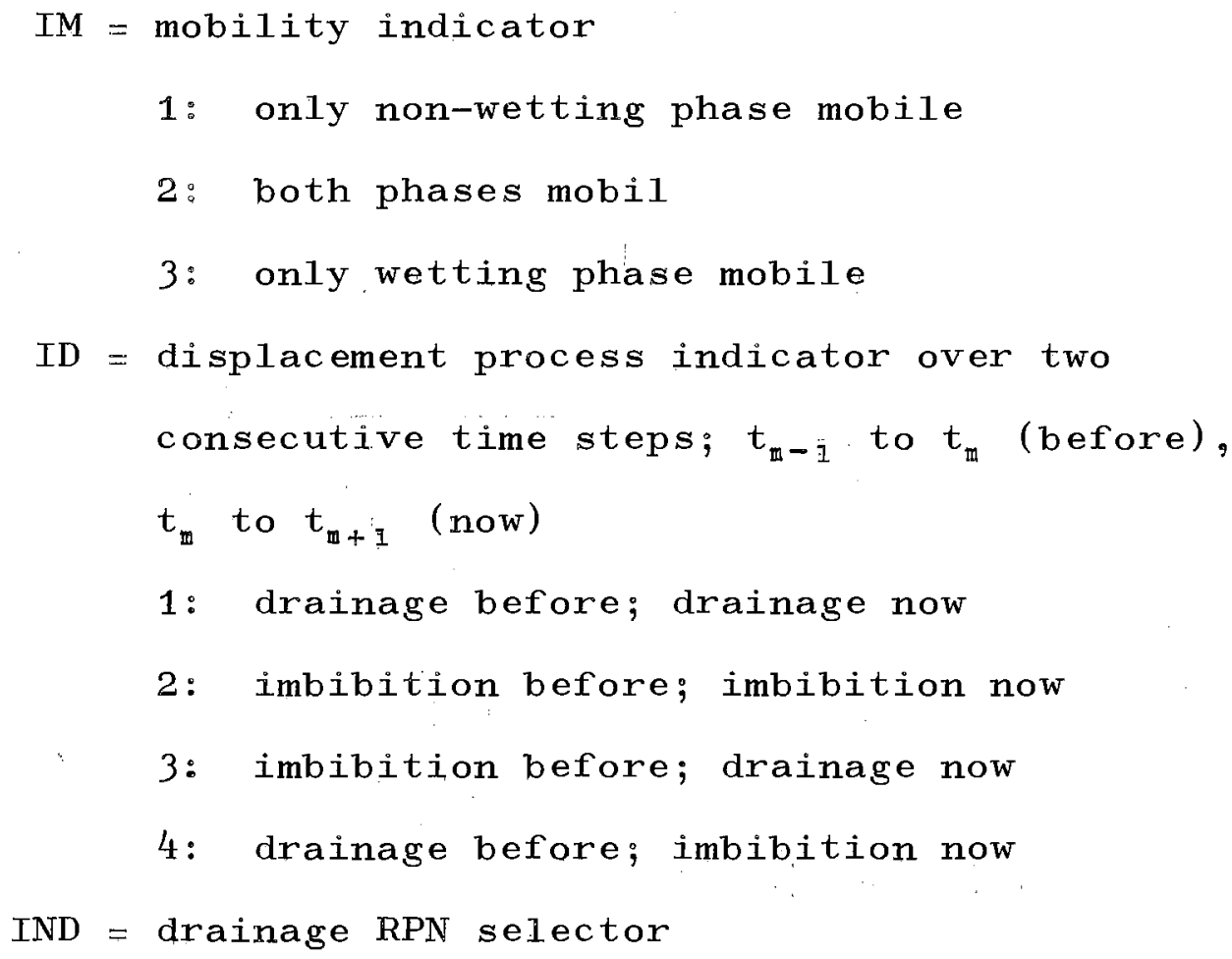




$$
\begin{aligned}
& \text { IWD }=\text { drainage RPW selector } \\
& \text { INI }=\text { imbibition RPN selector } \\
& \text { IWI }=\text { imbibition RPW selector }
\end{aligned}
$$

such that,

IND, IWD =

1: RP values on bounding drainage curves

2: RP values on intermediate drainage scanning curves branching off the bounding imbibition curves

3: RP values on intermediate drainage curves branching off scanning imbibition curves INI, IWI =

1: RP values on bounding imbibition curves

2: RP values on intermediate imbibition scanning curves branching off bounding drainage curves

3: RP values on intermediate imbibition scanning curves branching off scanning drainage curves,

the algorithm, named Hystrack (Figure 12) was developed to: 1) update the saturation history, and 2) select the set of appropriate equations yielding time level $t_{m+1}$ values of $R P$ and PC for use at time level $t_{w+2}$. All indicators are understood to carry subscripts ( $i, j, 1)$.

Since the economy of core storage is important in digital computers, a universal indicator (IU) was used to replace the state indicators in the computer program: 

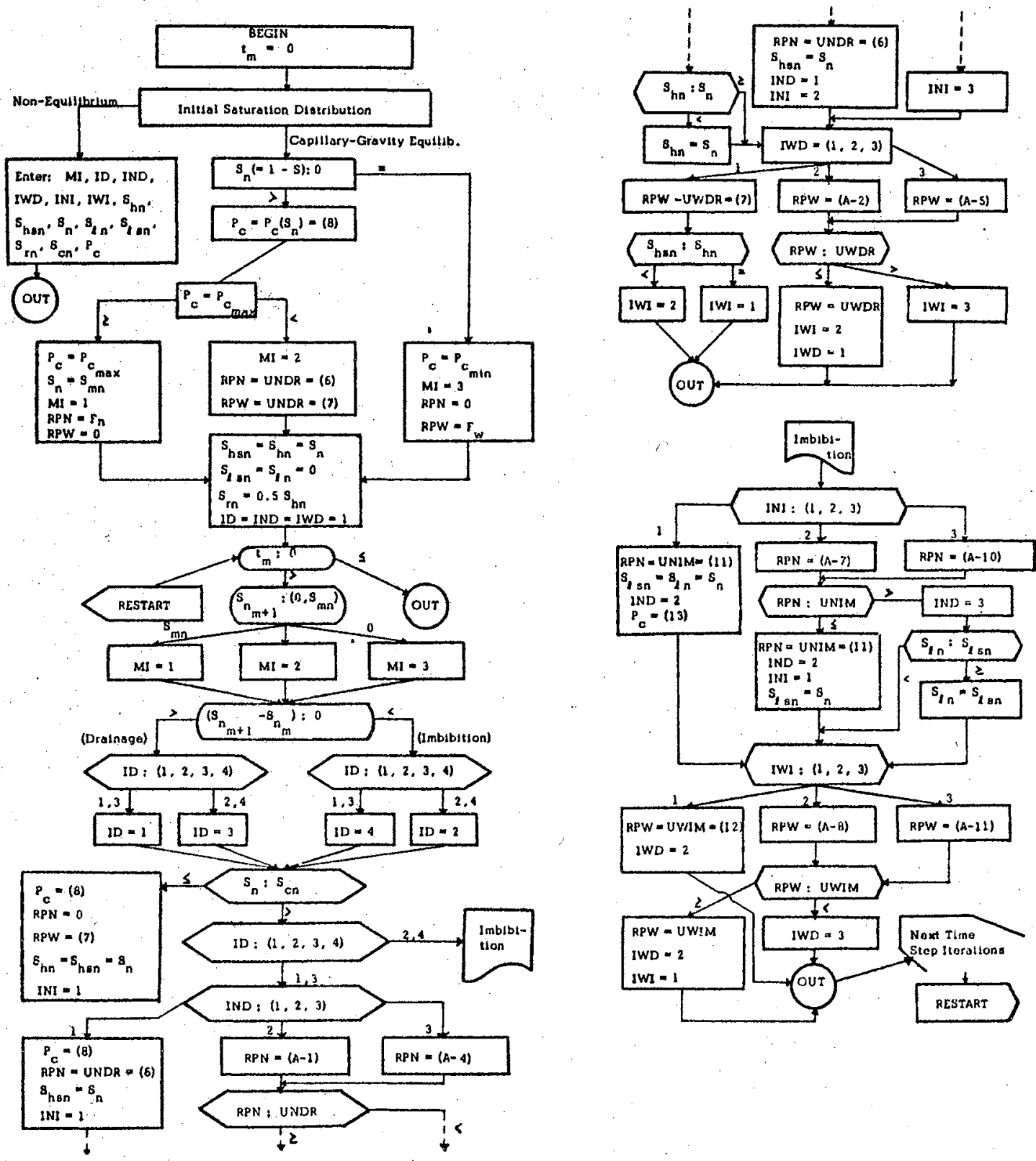

Figure 12. Flow Chart for Hystrack Algorithm 


$$
\begin{aligned}
\mathrm{IU}= & (\mathrm{IM}) 10^{5}+(\mathrm{ID}) 10^{4}+(\mathrm{IND}) 10^{3} \\
& +(\mathrm{INI}) 10^{2}+(\mathrm{IWD}) 10+(\mathrm{IWI}) \\
\mathrm{IM}= & \Psi\left(\mathrm{IU} \times 10^{-5}\right) \\
\mathrm{ID}:= & \Psi\left(\mathrm{IU} \times 10^{-4}\right)-10 \Psi\left(\mathrm{IU} \times 10^{-5}\right) \\
\mathrm{IND}= & \Psi\left(\mathrm{IU} \times 10^{-3}\right)-10 \Psi\left(\mathrm{IU} \times 10^{-4}\right) \\
\mathrm{INI}= & \Psi\left(\mathrm{IU} \times 10^{-2}\right)-10 \Psi\left(\mathrm{IU} \times 10^{-3}\right) \\
\mathrm{IWD}= & \Psi\left(\mathrm{IU} \times 10^{-1}\right)-10 \Psi\left(\mathrm{IU} \times 10^{-2}\right) \\
\mathrm{IWI}= & \mathrm{IU}
\end{aligned}
$$

where $\Psi$ represents the type transfer function ENTIER of the Extended Algol computer programming language. Figure 13 shows the loci of RPN calculated by HYSTRACK.

Conditions prevailing in the prototype reservoir, or lack of appropriate data, or simulation policy, or any combination thereof, may permit the use of hysteresis envelopes only, without scanning loops. A simplified version of HYSTRACK algorithm, SEMHYSTRACK was used for updating the reduced set $\left[S^{*}\right]$ and generating $R P$ and $P C$ loci on the basis of dynamic hysteresis envelopes without scanning loops, whenever simulation conditions did not require full rigor. 


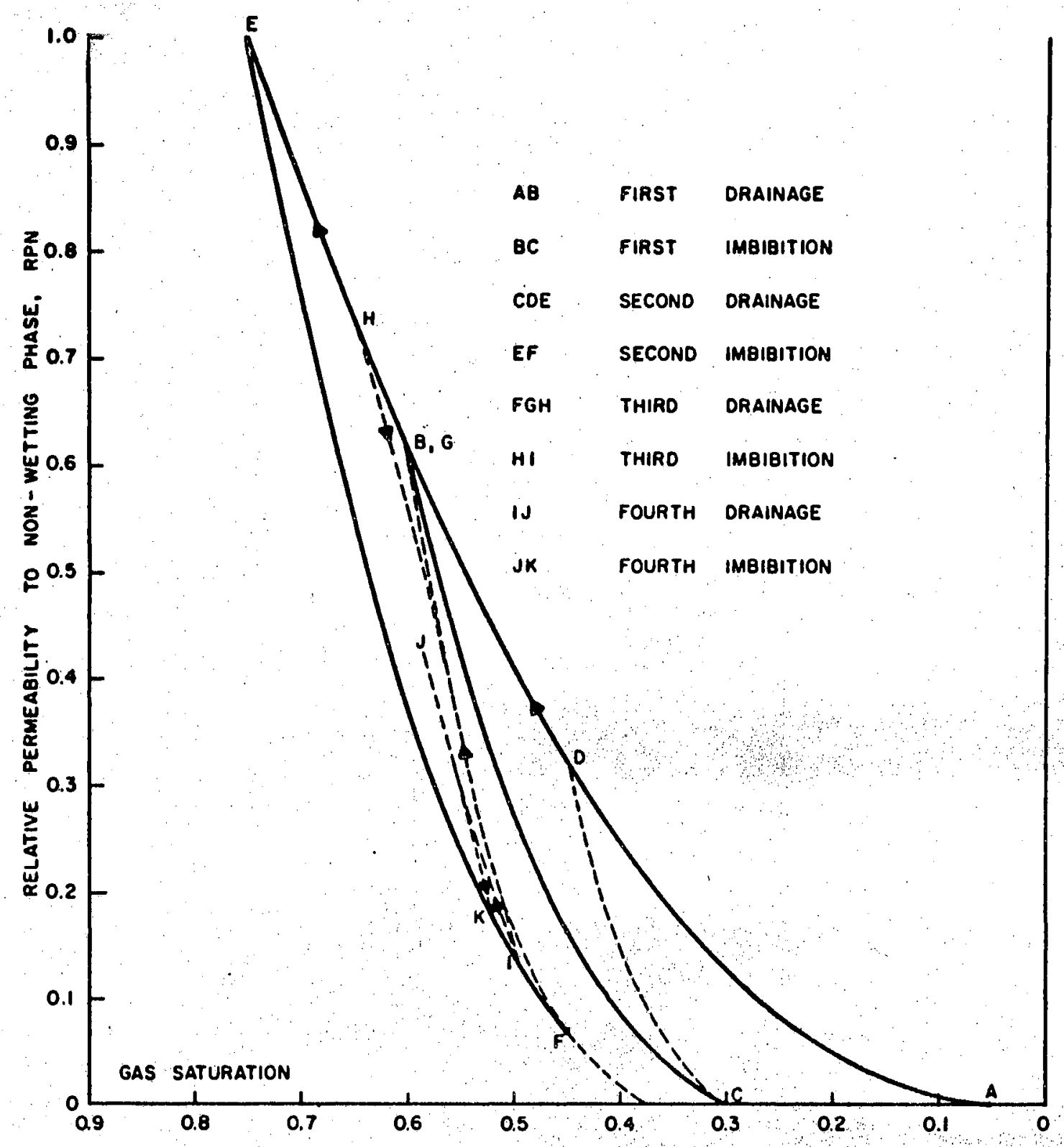

Figure 13. Loci of Non-Wetting Phase Relative Permeability Calculated by Hystrack Algorithm 


\section{APPENDIX D}

\section{PARTIAL INTEGRATION OF EQUATIONS OF TWO-PHASE FLOW--VERTICAL EQUILIBIUM (VE)}

The limiting vertical equilibrium (VE) concept, first investigated by Coates (8), permits the calculation of limiting pseudo-capillarity and relative permeability curves. In this dissertation, these pseudo-saturation functions played a very important role in reducing the scope of sensitivity studies. A recent paper by J. Martin (73) presents a rigorous method of partial integration of multi-phase flow equations, together with a theoretical foundation of $\mathrm{VE}$ condition.

When VE condition obtains in a reservoir flow system,

$$
\begin{aligned}
& \frac{\partial \Phi_{E}}{\partial_{z}}=\frac{\partial \Phi_{w}}{\partial_{z}}=0 \\
& P_{c x, y, z}=P_{x_{x y y}^{*}}^{*}-\cos \psi \int_{0}^{Z} \Delta \rho d z
\end{aligned}
$$

where,

$$
\begin{aligned}
P_{x, y}^{*}= & \text { value of capillary pressure at any areal } \\
& \text { point }(x, y) \text { on a reference surface. } \\
\Delta \rho= & \rho_{g}-\rho_{w}, \text { difference in specific weights, } \\
& \text { psilft. }
\end{aligned}
$$




$$
\Downarrow \quad=\text { dip angle. }
$$

The $z$ coordinate is oriented in the dip-normal direction and measures the distance below the reference surface. For sake of convenience, consider the reference plane to pass through the midpoints of all dip-normal line segments crossing the reservoir rock. Given any dip-normal saturation gradient $S_{w}(z)$ within a grid block and laboratory relative permeability curves, volumetrically averaged (or pseudo) saturation and effective relative permeabilities, for flow parallel to the $X-Z$ plane at any areal point on the reference surface, are obtained from:

$$
\begin{aligned}
\hat{S}_{w} & =\int_{-h / 2}^{h / 2} \varphi(z) S_{w}(z) d z / \int_{-h / 2}^{h / 2} \varphi(z) d z \quad(D-3) \\
\hat{k}_{r_{z}} & =\int_{-h / 2}^{h / 2} k_{x y}(z) k_{z_{g}}(z) d z / \int_{-h / 2}^{h / 2} k_{x y}(z) d z \quad(D-4) \\
\hat{k}_{r_{w}} & =\int_{-h / 2}^{h / 2} k_{x y}(z) k_{y_{w}}(z) d z / \int_{-h / 2}^{h / 2} k_{x y}(z) d z
\end{aligned}
$$

where porosity $\varphi$ and base permeability on the $x-y$ plane vary with $z$ in stratified reservoirs. The saturation $S_{w}$ and the relative permeabilities are indirect functions of $z$. When VE condition occurs, Equation (D-2) yields:

$$
\mathrm{d} \mathbf{z}=-\mathrm{d} \mathrm{P}_{\theta} / \Delta \rho \cos \psi
$$

Replacing the variable of integration dz by $d_{e}$, 
pseudo-relative permeability and capillary pressure functions are defined as:

$$
\begin{aligned}
& S_{w}=c \int_{a}^{b} \varphi(z) S_{w} d P_{\odot} / \int_{-h / 2}^{h / 2} \varphi(z) d z \\
& \hat{k}_{r_{g}}=c \int_{a}^{b} k_{x / y}(z) k_{r_{s}} d P_{o} / \int_{-h / 2}^{h / 2} k_{x y}(z) d z \\
& \hat{\mathbf{k}}_{\mathbf{r}_{w}}=c \int_{a}^{b} k_{x y}(z) k_{y_{w}} d P_{\theta} / \int_{-h / 2}^{h / 2} k_{x y}(z) d z
\end{aligned}
$$

where

$$
\begin{aligned}
& \mathrm{a}=\mathrm{P}_{\theta}^{*}-\Delta \rho \cos \psi \frac{\mathrm{h}}{2}=\mathrm{P}_{\mathrm{\theta}}^{*}-\frac{\Delta \mathrm{P}_{\mathrm{\theta}}}{2} \\
& \mathrm{~b}=\mathrm{P}_{\theta}^{*}+\Delta \rho \cos \psi \frac{\mathrm{h}}{2}=\mathrm{P}_{\mathrm{c}}^{*}+\frac{\Delta \mathrm{P}_{\theta}}{2} \\
& \mathrm{c}=1 / \Delta \rho \cos \psi \cdot
\end{aligned}
$$

The above equations represent $\hat{k}_{\delta_{g}}\left(\hat{S}_{w}\right), \hat{k}_{r_{w}}\left(\hat{S}_{w}\right)$, and $P_{0}^{*}\left(S_{W}\right)$ curves which reflect the dip-normal stratification and saturation distribution, under VE configuration.

When the prototype reservoir is homogeneous with constant dip-angle and thickness, and saturated with two immiscible fluids of negligible compressibility, the pseudorelationships (D-6 through D-8) are applicable over the entire reservoir. Otherwise, with areal variation of stratification, dip angle, anistropy in $x-y$ plane, thickness, and specific weight differences, separate 
pseudo-curves are required for each areal grid point representing a grid block.

The treatment of partial penetration of a well into a given block is fairly straightforward if vertical equilibrium can be assumed. In this case, the position of the center of the completion interval of the well relative to block height (or thickness) h is specified, and the relative permeability curves representing the total block adjusted to represent the completion interval only. Otherwise, the best approach appears to be the study of typical (or even individual wells) with detailed (small block dimensions) numerical simulators to determine saturation-rate relationships for well blocks such that gas or water will not move, and to adjust the RP curves accordingly. 


\section{APPENDIX E \\ PARAMETRIC SENSITIVITY STUDIES OF RP AND PC IN RESERVOIR MODELING}

The following data was used, except, as indicated, throughout the sensitivity studies:

$$
\begin{aligned}
& \lambda_{n}=1-2 ; \lambda_{k}=1-4 ; \\
& \omega_{n}=\omega_{M}=\omega_{c}=2 ; S_{m}=0.8 \\
& S_{c n}=0.1 \text { or } 0.05 \text { or } 0.01 \\
& S_{n n}=\left[\begin{array}{lll}
S_{n} & \text { when } & S_{n} \leq S_{0 m} \\
0.5 & S_{n n} & \text { when } S_{n}>S_{e n}
\end{array}\right. \\
& \Delta x=\Delta y=1000 \mathrm{ft} \cdot ; \Delta z=10 \mathrm{ft} . \\
& \varphi=0.2 ; \mathrm{k}_{\mathrm{a}_{\mathrm{g}}}=500 \mathrm{md} \cdot ; \mathrm{T}_{\varepsilon}=60^{\circ} \mathrm{F} \\
& P_{s}=780-830 \text { psi range at equilibrium } \\
& \rho_{n}=50 \mathrm{MSCF} / \mathrm{bbl} ; \rho_{n}=62.41 \mathrm{bf} \mathrm{ft}^{3} .
\end{aligned}
$$

Case of Gas Injection Into a Thin, Virgin Aquifer

Simulation Runs B1, B2, B3, B4

In this case, the $11 \times 11 \times 1$ grid system shown in Figure 10 was employed. The internal boundary conditions 
(or excitation functions) consisted of the explicit constant terminal injection rate of $1000 \mathrm{MSCF} / \mathrm{D} /$ well in blocks $(5,5),(7,5),(5,7),(7,7)$. The aquifer was assumed to be continuous to infinity, beyond the area spanned by the grid network; hence, Carter-Tracy type water influx-efflux conditions were used as external boundary conditions. In all four of the runs, the curvilinear capillarity data shown in Figure 14 was applied.

Simulation Run B1 was made with $\lambda_{n}=2, \lambda_{n}=4$, and $S_{e_{n}}=$ 0.1. Saturation distribution for simulation time level of 763 days is shown in Table III. After 763 days of injection, block $(5,5)$ water saturation was 0.6780 . At this saturation, the mobility ratio $\left(k_{n n} \mu_{w} / k_{n w} \mu_{n}\right)$ is 65 to 1 in favor of the gas phase. This explains the rather high water saturation remaining in the input block. With $S_{n}=0.1$, gas cannot move out of one block and enter another, until the water saturation of the block with higher gas potential is less than 0.90. At a water phase saturation of 0.85 , the mobility ratio is 0.975 ; hence, at that saturation gas can move as easily as water from block-to-block. High water saturations observed in gas invaded blocks of the grid system, at $t=763$ days, are caused by the high mobility of gas at low gas saturations. In this 10 feet thick system continuously supplied by four wells, the viscous forces dominate the capillary-gravity forces, and coupled with the high viscosity contrast, sustain an inefficient displacement process. 


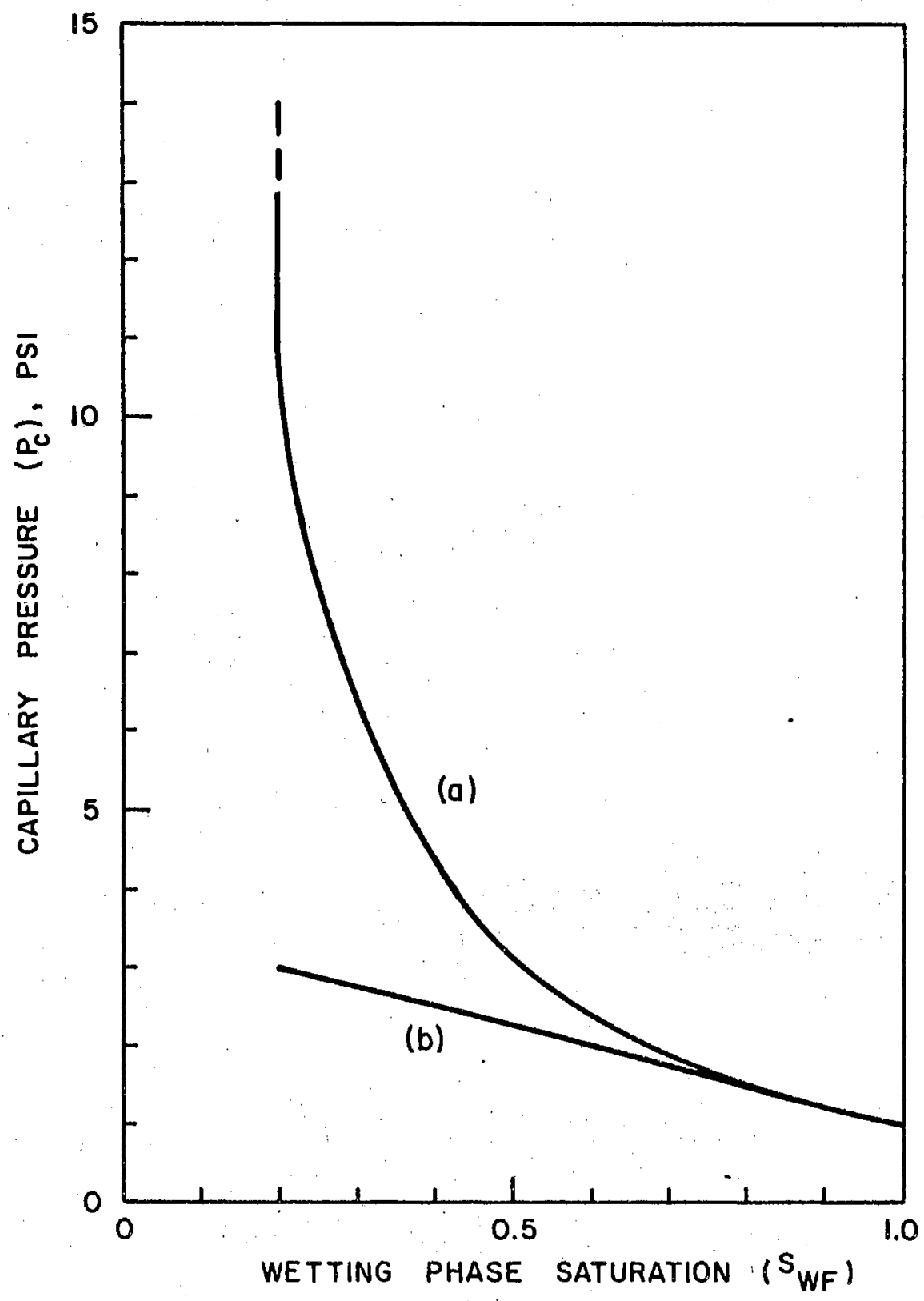

Figure 14. Linear and Curvilinear Capillary Pressure Curves Used in Injection and Production Simulation Studies 
TABLE III

SATURATION $\left(\mathrm{S}_{W}\right)$ MAP FOR RUN B1 AT THE STMULATION TIME LEVEL OF 763 DAYS

\begin{tabular}{llllll}
\hline $\mathrm{I}=\mathrm{J}=1$ & & & 0.99 & 0.92 & 1.00 \\
& & 0.99 & 0.88 & 0.81 & 0.97 \\
0.99 & 0.89 & 0.86 & 0.80 & 0.78 & 0.90 \\
0.92 & 0.81 & 0.78 & 0.73 & $0.67^{*}$ & 0.80 \\
1.00 & 0.97 & 0.90 & 0.85 & 0.80 & 0.95 \\
\hline
\end{tabular}

*Input well location 
Since the gas phase has high mobility even at low gas saturations, $S_{0}$ becomes a very important parameter in thin reservoir studies. The lower is the $S_{e}$, the higher is the RPN - hence mobility - to gas at a given saturation. To show the impact of $S_{e}$, simulation runs $B 2$ with $S_{0}=0.05$ and $B 3$ with $S_{0}=0.01$ were made. Figures 15 and 16 display water saturation versus simulation time relationships of the key blocks corresponding to three values of $\mathrm{S}_{\mathrm{c} n}=0.1,0.05$, and 0.01 . In well blocks, higher $S_{n}$ values are obtained with higher values of $S_{0}$, throughout the simulation runs, as expected from mobility considerations. The converse is true for edge blocks; that is, the lower the $\mathrm{S}_{\mathrm{e}}$, the higher is the gas saturation. This is entirely as expected. The more mobile is the gas, the farther it will move from the input blocks, at a given time and for a given injection volume. The volumes of gas injected for Runs B1, B2, and B3 were kept the same for comparison.

Runs B1, B2, B3 demonstrate that $S_{\text {en }}$ is the most important parameter in simulating gas injection. into a virgin aquifer. $S_{G n}$ is also first order important in hysteretic, cyclic flow. For a given volume of gas injected, the higher is the $S_{o n}$, the smaller is the size of the gas bubble. Hence, some blocks containing gas would not have undergone gas invasion, had $S_{c n}$ been higher than the value used in the simulation study, and no gas would have been trapped in such blocks upon imbibition. The reader is reminded that, in this work, trapped gas saturation of a block is a function 


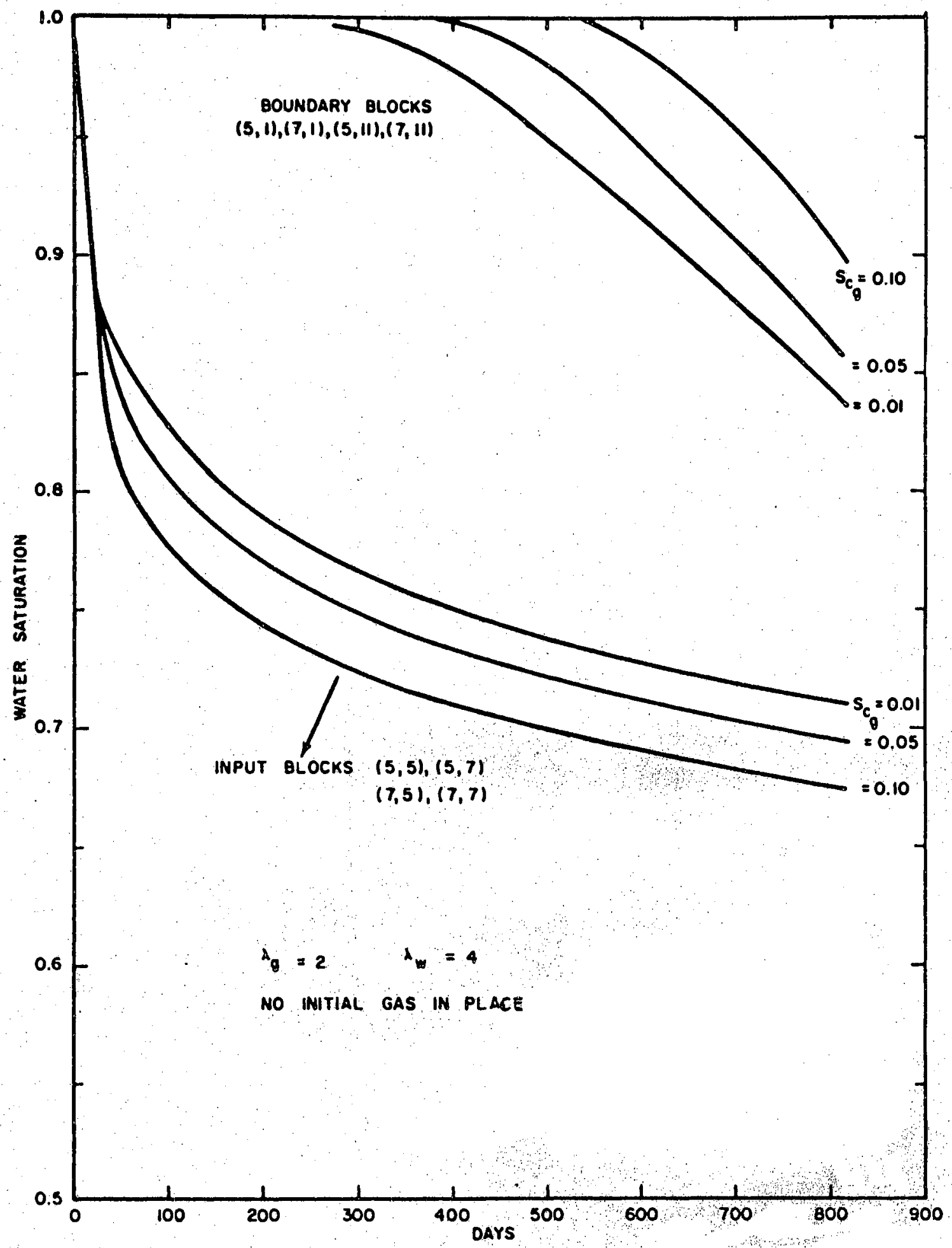

Figure 15. Water Saturation Versus Simulation Time Plots for Runs B1, B2, and B3 [B1ocks $(5,1)$ and $(5,5)]$ 


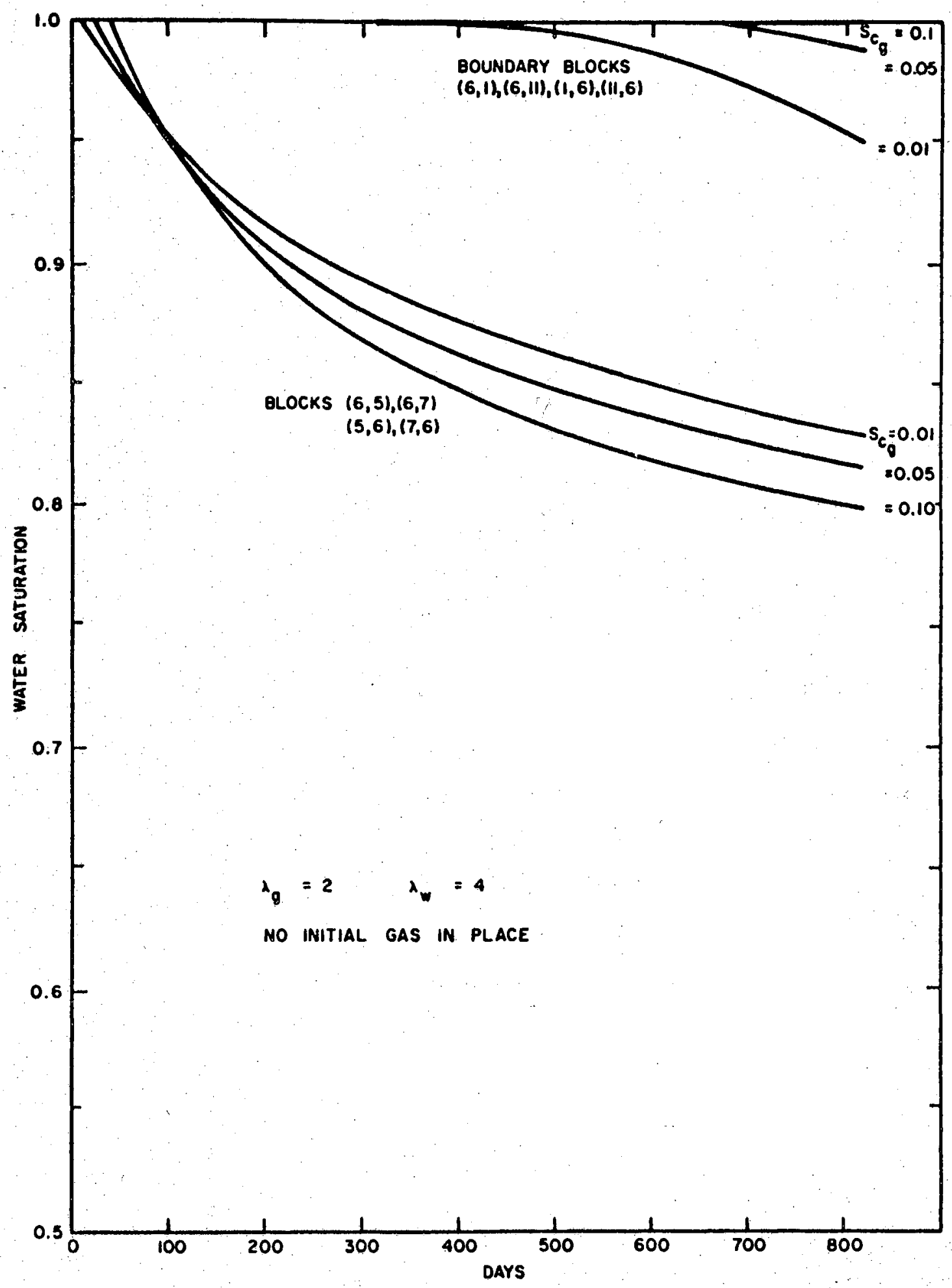

Figure 16. Water Saturation Versus Simulation Time Plots for Runs B1, B2, and B3 [Blocks $(6,1)$ and $(6,5)]$ 
of the historical peak gas saturation of that block. The impact of $S_{c}$ decreases, however, as the viscosity contrast between the two fluids decreases.

The difference in the saturations of the adjacent blocks $(5,1)$ and $(6,1)$, caused by well interference (see Figure 10), is amplified by the rather coarse grid system applied. For example, at $t=763$ days and for $s_{c}=0.01$, $S_{k}$ in blocks $(5,1)$ and $(6,1)$ were 0.835 and 0.995 , respectively. Furthermore, the $S_{w}$ contrast $(0.895$ and 1.00$)$ increased as $S_{o n}$ increased. If a more detailed grid network were used, these differences would have been considerably smoothened. The simulation technologist should expect higher saturation "errors" in frontal development as the value of $S_{0}$ and block size increase.

Run B4 was made with $\lambda_{n}=\lambda_{w}=1$ and $S_{e n}=0.1$. When the two interference exponents are equal to unity, RP curves become straight lines. Water phase saturation versus simulation time relationships of various key blocks are shown in Figure 17. Run B1 data is also presented as dotted lines for comparison. The impact of $\lambda$ on the saturation distribution is similar to that of $S_{n}$; that is, as $\lambda$ decreases, $R P$ values increases, both gas and water become more mobile in two-phase saturated blocks; hence, the gas "bubble" spans a larger region of the reservoir, for a given injection volume.

Dynamic pressure versus simulation time relationships of the gas input blocks and the boundary blocks, obtained 


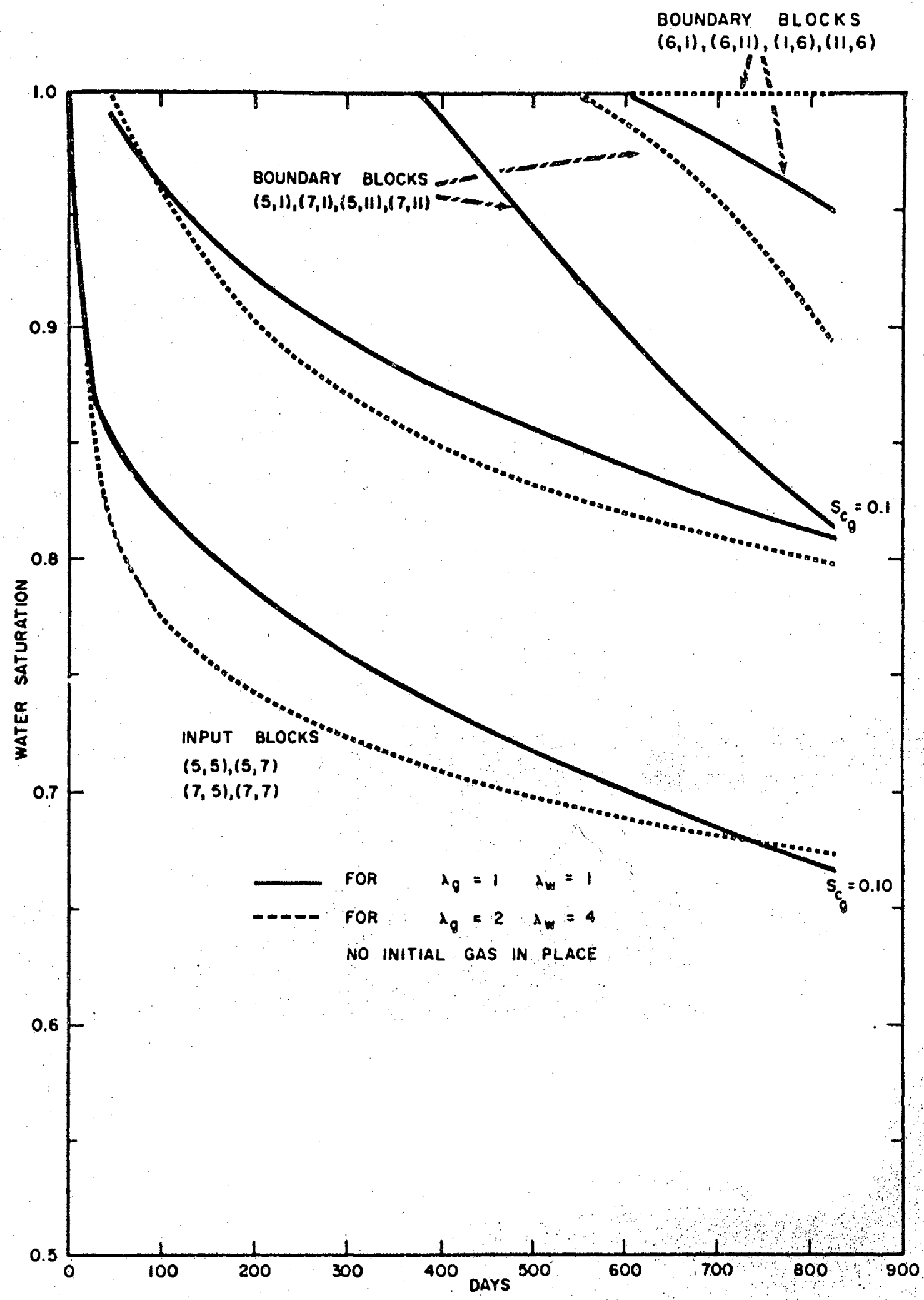

Figure 17. Water Saturation Versus Simulation Time Plots for Run B4 [Blocks $(5,1)$, $(5,5)$, $(6,1)$, and $(6,5)]$ 
from the simulation Runs B1 and B4, are presented in Figure 18. Well block pressure increases with the interference exponent, $\lambda$; because, the higher values of $\lambda$ produce lower phase mobilities within the two-phase flow zone. The converse is true for the boundary blocks, as indicated by Figure 18. Higher input block dynamic pressures, at a given saturation and simulation time, simply mean that more gas was stored in the input blocks due to compression; hence, water efflux rate into the aquifer and the grid boundary pressure are lower. As the gas front approaches the grid boundary, the pressure differences in boundary blocks, due to $\lambda$, decrease. Interference coefficients, $F_{*}$ and $F_{n}$, are direct multipliers of $R P$ relations. The lower are $F_{w}$ and $F_{n}$, the lower are the phase mobilities in direct proportions, within the two-phase zone.

To summarize, the saturation and pressure distribution calculations for the case of initial gas injection into a thin virgin aquifer are sensitive to the critical gas saturation, the phase interference exponent, and the phase interference coefficient, in that order, as they govern the mobility of the two fluids. However, the differences in these distributions, due to $\lambda$ and $S_{e n}$, may well be within the computational accuracy desired. This is usually the case when the geometry of the prototype reservoir is not well-known. For example, dynamic pressure variation due to $\lambda$ in well block $(5,5)$ is about $3.5 \%$, based on the higher value. 


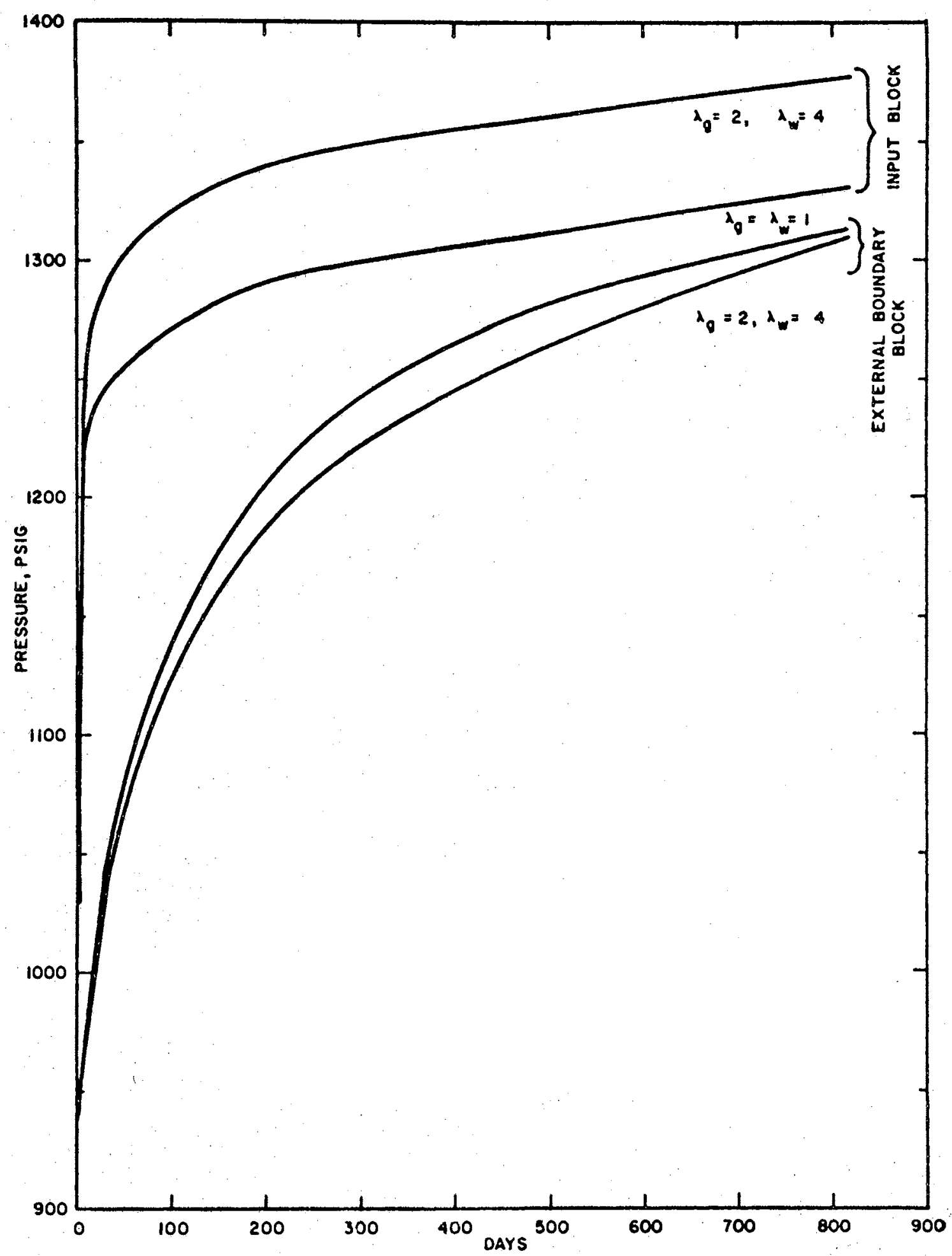

Figure 18. Dynamic Block Pressure Versus Simulation Time Plots for Runs B1 and B4 
For gas-water systems, PC is generally very low, except near $S_{r w}$. Therefore, it is the slope of the capillary pressure curve, not the capillary pressure leve1, that influences the computations, for $S_{k}>S_{k}$. The change in the saturation distribution with simulation time, for an initial drainage process, is such that the drainage PC curve, Figure 14(a), can be replaced with a straight line, Figure 14(b). The effects of the size of the grid area and the size of the grid blocks on saturation-pressure distributions were also investigated. The findings are reported in this Appendix.

$$
\begin{gathered}
\text { Case of Gas Injection Into a Partially Gas } \\
\text { Saturated, Thin Aquifer at Initial } \\
\text { Capillary-Gravity Equilibrium }
\end{gathered}
$$

Simulation Runs B5 and B6

Simulation Runs B5 and B6 were made to investigate the effects of the interference exponent, $\lambda$, governing phase mobility, on saturation and pressure distributions, when an equilibrium gas bubble was excited by gas injection and forced to grow in a thin aquifer. Original gas-water contact was placed at 174 feet above the center of the reference block $(1,1,1)$. Grid system and boundary conditions used for Runs B1 through B4 were again applied. Run B5 was made with linear bounding RP curves $(\lambda=1)$. Run B6 employed curvilinear RP, with $\lambda_{B}=2$ and $\lambda_{w}=4$.

Saturation profiles at the beginning and at the end of 
the Runs $\mathrm{B} 5$ and $\mathrm{B} 6$ are presented in Table IV. The response to $\lambda$ was the same as the response in Runs $B \lambda$ through $B 4$; however, the saturation differences produced by $\lambda$ were very small near the gas injection points where gas saturation was relatively high. The largest $S_{w}$ variation in response to $\lambda$ was observed in edge block $(5,1) ; 0.8864$ for Run B5 and 0.9695 for Run 6. The pressure variation in response to $\lambda$ in well block $(5,5)$ was approximately $2.7 \%$, which is within the desired accuracy for most applications.

The distributions computed by the simulator for the case of constant rate gas injection into a thin aquifer, with or without initial gas in place, showed that the, maximum variation due to relative permeability, were about $8 \%$ of pore volume for saturation and $3.5 \%$ for pressure, relative to the simulation conditions for Runs B5 and B6. For thin reservoir systems with higher pressure levels, these percentages would be smaller. Conversely, for systems with lower base permeability the errors due to RP data would be higher.

Case of Constant Terminal Rate Production From a Thin Reservoir-Aquifer System, Initially at Capillary-Gravity Equilibrium

Simulation Runs C1, C2, C3, D1

In this case, the grid system (see Figure 10) and rock-fluid properties used in Simulation Run group B were again applied. Original gas-water contact was placed at 
TABLE IV

SATURATION ( $\left.S_{w}\right)$ PROFILES FOR RUNS B5 AND B6 AT THE SIMULATION TIME LEVEL OF 763 DAYS

\begin{tabular}{cccr}
\hline$J(I=5)$ & $S_{w}^{\prime}($ Initial $)$ & $S_{w}^{\prime}(B 5)$ & $S_{w}^{\prime}(B 6)$ \\
1 & 1.000 & 0.886 & 0.969 \\
2 & 1.000 & 0.856 & 0.832 \\
3 & 1.000 & 0.838 & 0.787 \\
4 & 1.000 & 0.722 & 0.723 \\
5 & 0.338 & 0.321 & 0.337 \\
6 & 0.236 & 0.237 & 0.235 \\
$\lambda_{n}=\lambda_{w}=1$ for Run B5: & $\lambda_{n}=2, \lambda_{w}=4$ for B6; \\
and $S_{c n}=0.1$. & & \\
\hline
\end{tabular}


160 feet above the center of the reference block $(1,1,1)$. The internal boundary conditions consisted of the explicit constant terminal gas withdrawal rate of $1000 \mathrm{MSCF} / \mathrm{D} / \mathrm{We} 11$ from blocks $(5,5),(7,5),(5,7),(7,7)$. Water cut was calculated from the relation:

$$
Q_{w}=\frac{Q_{n} k_{r n}-\mu_{w} B_{n}}{k_{r w} \mu_{n} B_{w}}, \quad S T B / D / W e l l
$$

where $Q_{n}=1000 \mathrm{MSCF} / \mathrm{D}$.

Runs C1, C2, and C3 were made with the following values of parameters controlling the shapes of RP and PC curves:

\begin{tabular}{|c|c|c|c|c|c|c|}
\hline Run & $\underline{\lambda_{n}}$ & $\underline{\lambda_{x}}$ & $\underline{w}_{n}$ & $\underline{w}_{w}$ & $y$ & Drainage $P_{0}$ \\
\hline $\mathrm{C}_{1}, \mathrm{D} 1$ & 1 & 1 & 2 & 2 & 1 & curvilinear \\
\hline $\mathrm{C} 2$ & 2 & 4 & 2 & 2 & 1 & curvilinear \\
\hline C 3 & 2 & 4 & 2 & 2 & 1 & 1inear \\
\hline
\end{tabular}

Imbibition relative permeability to both phases, hence phase mobility, is higher for lower values of $\lambda$, with $w$ held constant.

Saturation profiles for these runs are given in Table V. A comparison of Run $\mathrm{C} 1$ and $\mathrm{C2}$ saturation distributions, at simulation time level of 313 days, revealed insignificant saturation differences. However, pressure comparison revealed significant sensitivity to $\lambda$ in blocks with high or full water saturation, even though saturation differences between runs $C 1$ and $C 2$ were negligible. This is entirely as expected, since water relative permeability, hence mobility, is very sensitive to $\lambda_{w}$. With $\lambda_{w}=1$, water phase is considerably more mobile, compared to $\lambda_{w}=4$ level. Higher 
mobility to water produces lower pressure gradients. Pressures in blocks $(5,5)$ and $(5,6)$ compared very closely, because in these blocks water saturation were near the residual level where RPW is insensitive to $\lambda_{\text {w }}$.

Lower values of $\lambda_{w}$ should produce higher water cuts for a given gas production rate. This is shown to be true in Figure 19, which is a plot of water cut versus simulation time. In Run $\mathrm{C} 1$ the production wells were found to be incapable of sustaining the explicit gas flow rate of 1000 MSCF/D/We1l at and beyond 373 days, whereas this incapability occurred at the time level of 433 days for Run C2. An examination of saturation distributions, at these failure times for each run, shows that the gas saturation of the blocks adjoining well blocks were at residual (or trapped) level; hence, water phase gushed into well blocks and increased the water cut of producing wells (Figure 19). An interesting paradox was observed in the saturation distributions of $t=373$ days and $t=403$ days for Runs $C_{1}$ and $\mathrm{C} 2$, respectively. How could a well placed in a block, such as block $(5,5)$, with a saturation of only $22-28 \%$ water, produce such tremendous quantities of water? The answer was very simple. The simulator base transmissibility was taken to be a "chord" between the centers of two adjoining blocks, and it was time independent. However, effective transmissibilities were saturation, hence, time dependent. When two adjoining blocks have a very large saturation contrast ( such as 0.66 versus 0.28 ) one is 


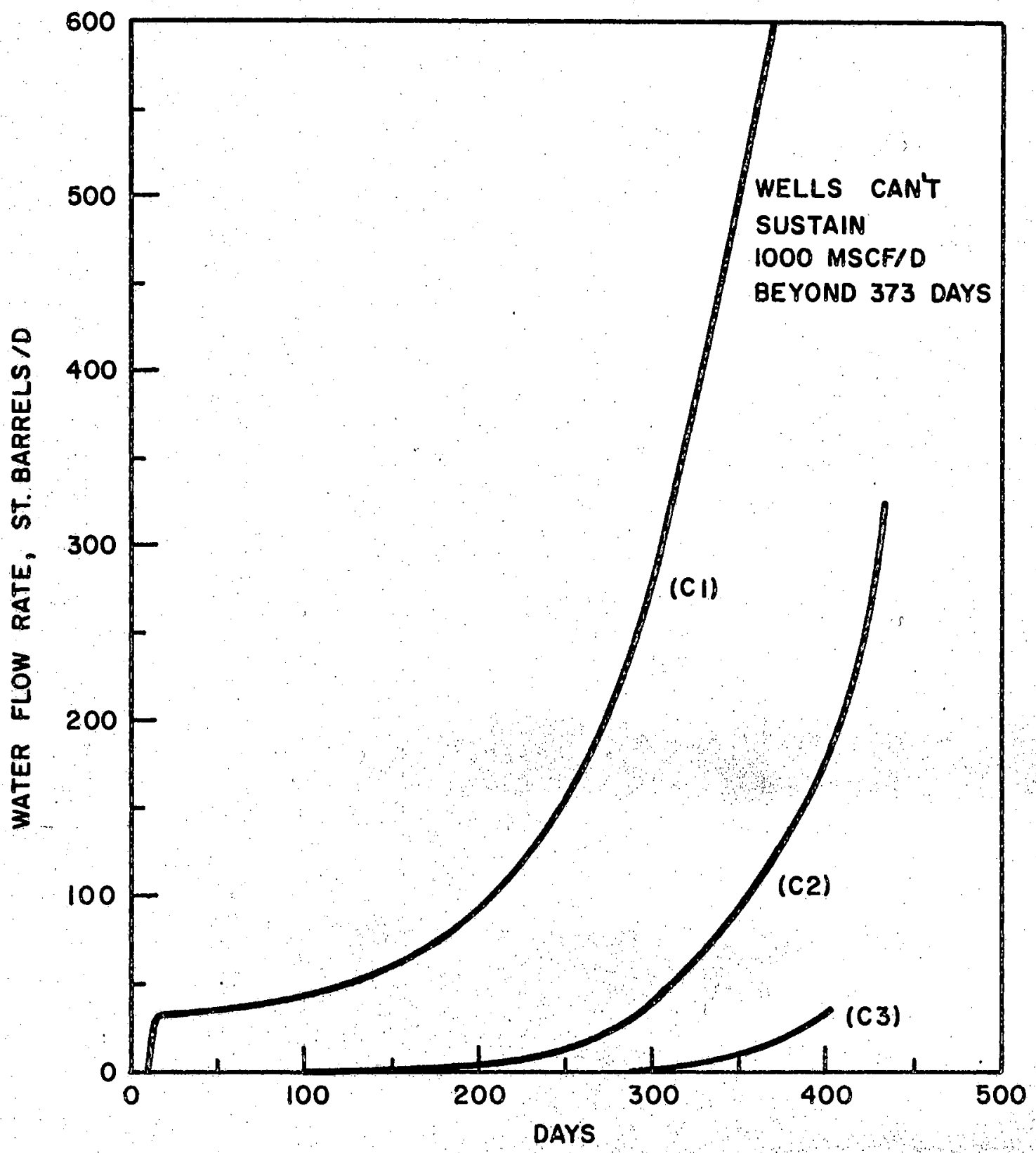

Figure 19. Water Cut Per Well in Simulation Runs C1, $\mathrm{C} 2$, and $\mathrm{C} 3$ 
confronted with a dilemma; which saturation should be used in applying an RP to the base permeability cord joining the two blocks? In these simulation runs the saturation of the block with higher potential was used. In this example, the key block would be $(5,4)$, where RPW is nearly unity. Now, the reason why block $(5,5)$ water saturation was still about 0.28 while large quantities of water entered this block, was that the producing well removed the water almost as fast as it came into the block.

This paradox can be circumvented by using the saturation of the producing we11 block to compute the we11's water output, and the saturations of the two blocks weighed equally for block-to-block transfer of the two fluids. However, for any two adjoining block pairs without we11s, the use of the saturation of the higher potential blocks was found to be quite satisfactory. Another reason for excessive water production from wells, in Runs $C_{1}$ and $C_{2}$, was that the imbibition RPW curve used in these runs, with $E_{w}=1$ was not realistic. The usual range for $E_{p}$ is 0.1 to 0.5 . Since $E_{w}$ is a direct multiplier of imbibition RPW, block-toblock water transfer rate would have been smaller with $\mathrm{E}_{\mathrm{w}}<1$. These runs served well to demonstrate that internal boundary conditions and those parameters governing the shape of imbibition RPW become very important as the gas bubble "collapses" toward the producing wells. Therefore, the neglect of the hysteresis in $R P$ and $S_{r n}$ could cause significant errors in phase mobility and entrapment, at the 
later stages of gas withdrawal from an aquifer-reservoir complex.

Run C3 was made with linear drainage capillarity data. (Figure 14b) In contrast to the group B injection simulation studies, PC data did influence the pressure and saturation distributions throughout the simulation run by affecting the initial gas-in-place and saturation distribution. With the initial gas-water contact and initial reservoir pressure held constant, the initial distribution is quite sensitive to PC data if the capillary-gravity equilibrium prevails. In Runs $\mathrm{C} 1$ and $\mathrm{C2}$, the calculated gas in place volume was 1.926 BSCF, whereas Run C3 initial gas volume was 2.157 BSCF. Water cut of one of the producing wells, for Run C3, is also shown in Figure 19. It is much lower than its counterparts for Runs $C_{1}$ and $C_{2}$. The explanation for the reduced water cut is fairly simple. The difference in initial gas in place volumes mentioned earlier is 231 MMSCF, which is better than one-half of the 403 MMSCF of produced gas.

\section{Simulation Run D1}

Run D1, which is similar to Run C1 in every aspect, excepting the production schedule, was made to investigate how a well's capability to produce deteriorated as the gas bubble collapsed toward the producing wells. Explicit terminal flow rate per well was specified to be 1000 MSCF/D of two-phase misture. Simultaneous gas and water flow 
rates computed by the simulator for Run D1 are plotted in Figure 20 and compared to Run $C_{1}$ performance. The initial distributions for Run D1 were the same as those of Run C1. Phase saturation profiles for Run D1 are shown in Table V. The most interesting observation on this run was that the gas flow rate per well declined from $1000 \mathrm{MSCF} / \mathrm{D}$ to 38 MSCF/D, over 823 days, as the gas bubble collapsed about the producing wells. The term collapsing gas bubble implies vanishing mobility to gas as gas saturation nears the residual (or trapped) level about the producing wells. Once again, the paradox described earlier was present in blocks $(5,4)$ and $(5,5)$; however, it was not as obvious as it was in Run C1. The main contribution of Run D1 was the demonstration of the fact that clever and realistic specification of the internal boundary conditions, hence the RP's of the blocks containing wells, are of first order importance in production simulation studies and accurate description of trapped gas volumes.

Case of Gas Injection-Withdrawal Operations in Multi-Layered or Thick Aquifer-Reservoir Systems

The sensitivity of saturation and pressure distributions in simulating multi-layered aquifer-reservoir systems, to RP and PC data, is dependent upon the sensitivity of the individual layers, with strata of high effective permeability dominating the over-all sensitivity. When individual 


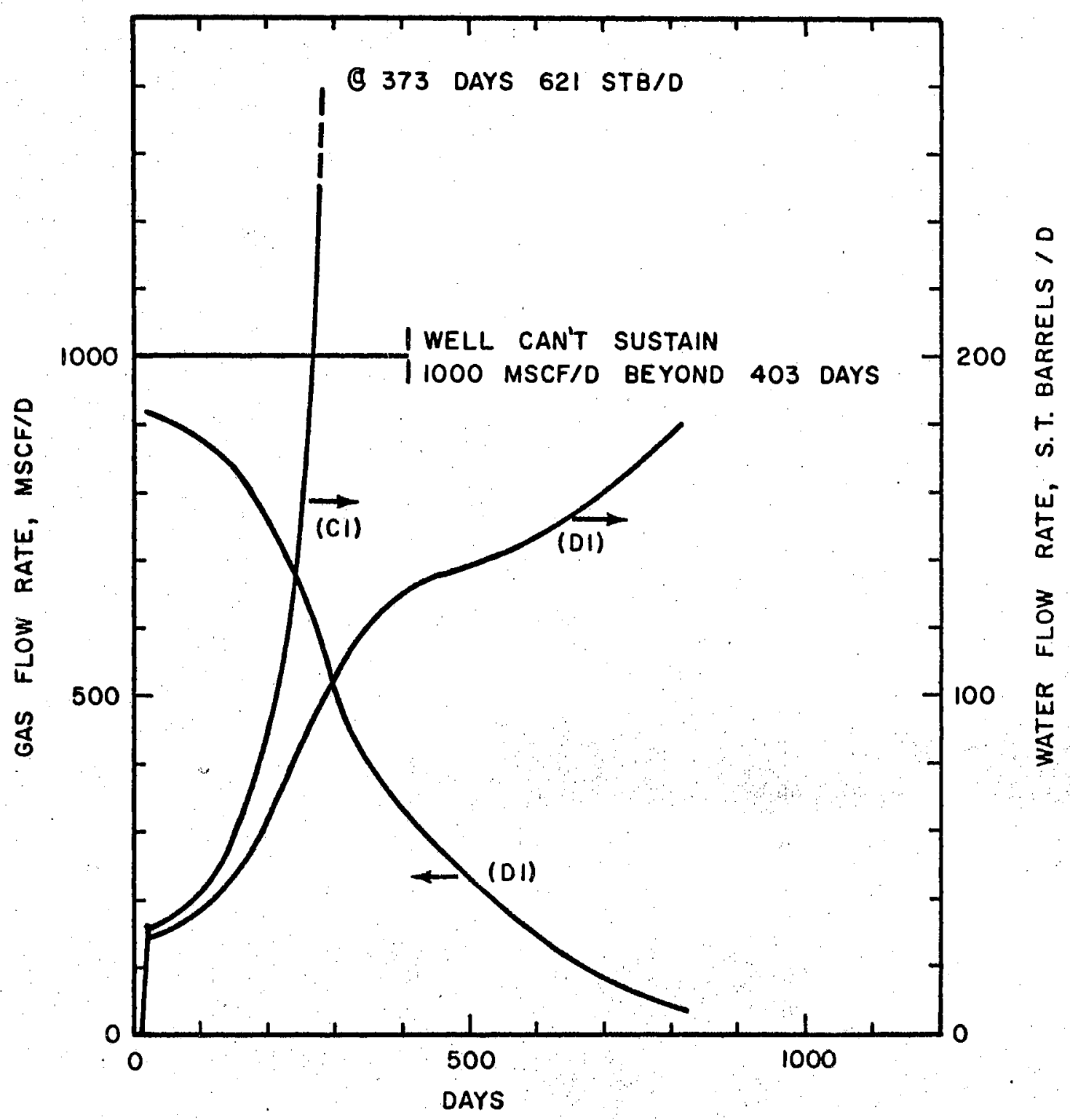

Figure 20. Production Schedules of Wells in Simulation Runs $\mathrm{C} 1$ and D1 
TABLE V

SATURATION $\left(S_{w}\right)$ PROFILES FOR RUNS $c_{1}, c_{2}, c_{3}$ AT STMULATION TIME OF 313 DAYS AND RUN D1 AT 823 DAYS

\begin{tabular}{|c|c|c|c|c|c|}
\hline$J(I=5)$ & $S_{w}($ Initial $)$ & $S_{w}\left(C_{1}\right)$ & $S_{W}(c 2)$ & $S_{W}(D 1)$ & $S_{w}\left(c_{3}\right)$ \\
\hline 1 & 1.000 & 1.000 & 1.000 & 1.000 & 1.000 \\
\hline 2 & 1.000 & 1.000 & 1.000 & 1.000 & 1.000 \\
\hline 3 & 1.000 & 1.000 & 1.000 & 1.000 & 1.000 \\
\hline 4 & 0.380 & 0.630 & 0.647 & 0.690 & $0.579(0.2) *$ \\
\hline 5 & 0.200 & 0.203 & 0.203 & 0.600 & $0.203(0.2) *$ \\
\hline 6 & 0.200 & 0.200 & 0.200 & 0.426 & $0.200(0.2) *$ \\
\hline \multicolumn{6}{|c|}{$\begin{array}{l}\lambda_{n}=\lambda_{w}=1 \text { for Runs } C 1 \text { and } D 1 ; \lambda_{n}=2, \lambda_{w}=4 \text { for Runs } C 2 \text { and } \\
C_{3} ; \text { linear PC curve for Run } C 3 ; \text { all runs with } \omega_{n}=w_{w}=2, \\
\gamma=1, S_{e n}=0.2, S_{n}=0.5, S_{n} ; * \text { denotes initial saturation. }\end{array}$} \\
\hline
\end{tabular}


layers are thin and/or the dip-normal base permeability is very low, dip-normal saturation gradients become negligible. In this case, the simulator performance is sensitive to RP and PC data to the extent presented in the previous sections of this chapter.

In the case of the single or multi-layered systems with appreciable thickness, dip-normal base permeability is of primary importance. Consider laboratory PC versus water saturation data shown in Figure 21 and the RP data presented in Figure 22. These figures describe PC, RPN, and RPW hysteresis envelopes. Equilibrium PC and RP curves for various depths of partial integration in dip-normal direction were computed by an auxiliary integration program. These are also shown in Figures 21 and 22 . The comparison of rock (dotted lines) and dip-normal equilibrium curves (solid lines) reveals that; if dip-normal equilibrium (or near equilibrium) prevails, the sensitivity of model performance to PC and RP data declines as the formation thickness increases, excepting the residual saturations. In other words, as the thickness increases, one has:

$$
\lambda_{n}^{*}, \lambda_{n}^{*}, \omega_{n}^{\prime} *, \omega_{n}^{*} \rightarrow 1, S_{0}^{*} * 0
$$

where * denotes simulator conditions. As a matter of fact, equilibrium drainage $P C$ and $R P$ curves can be represented by straight lines encompassing the residual and full saturations of the wetting phase for thicknesses larger than 10 feet. Similarly, straight lines spanning WF and NWF 

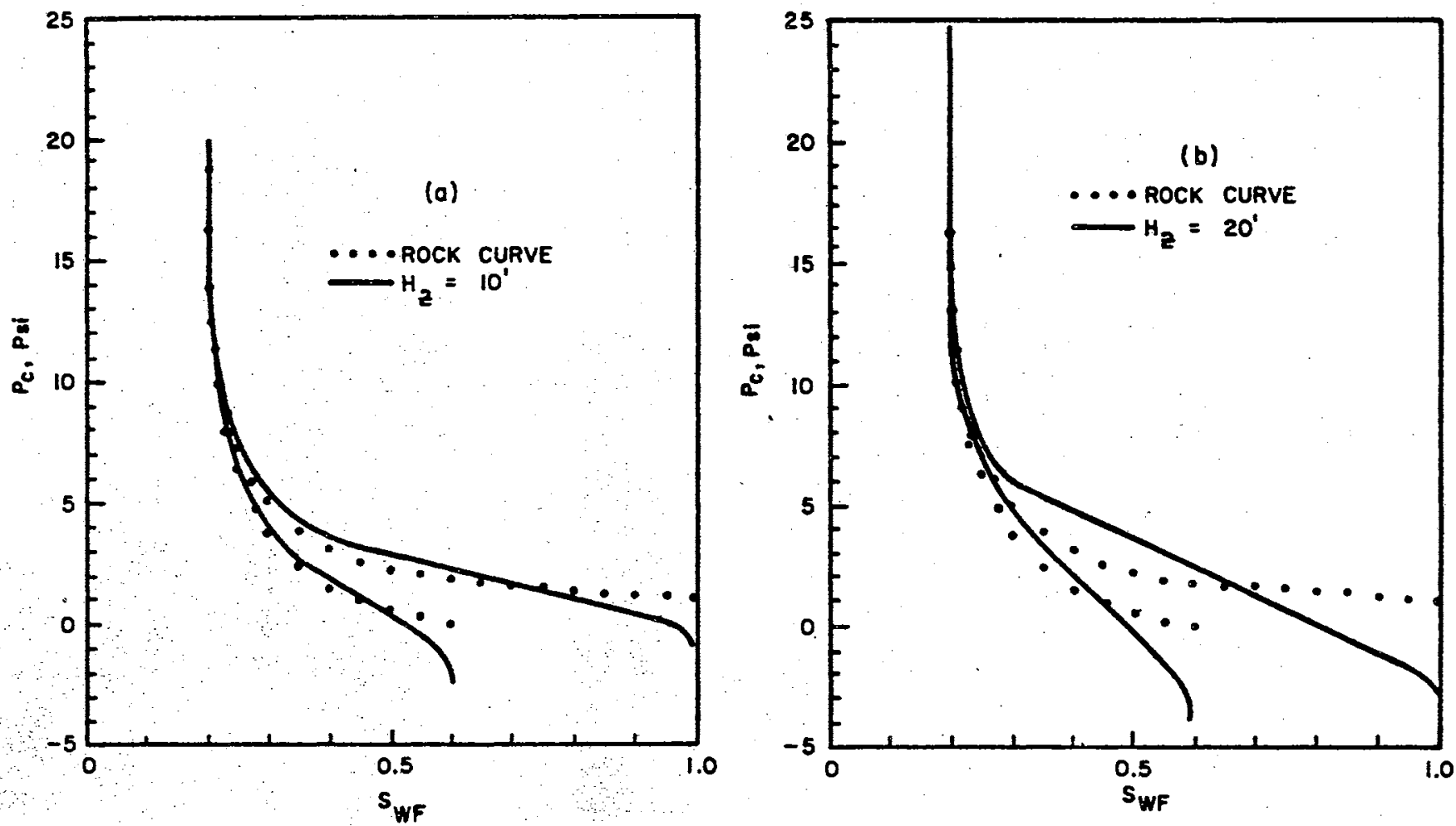

Figure 21. Sensitivity of Equilibrium Capillary Pressure to Depth of Partial Integration in Dip-Normal Direction, $\mathrm{H}_{2}$ 


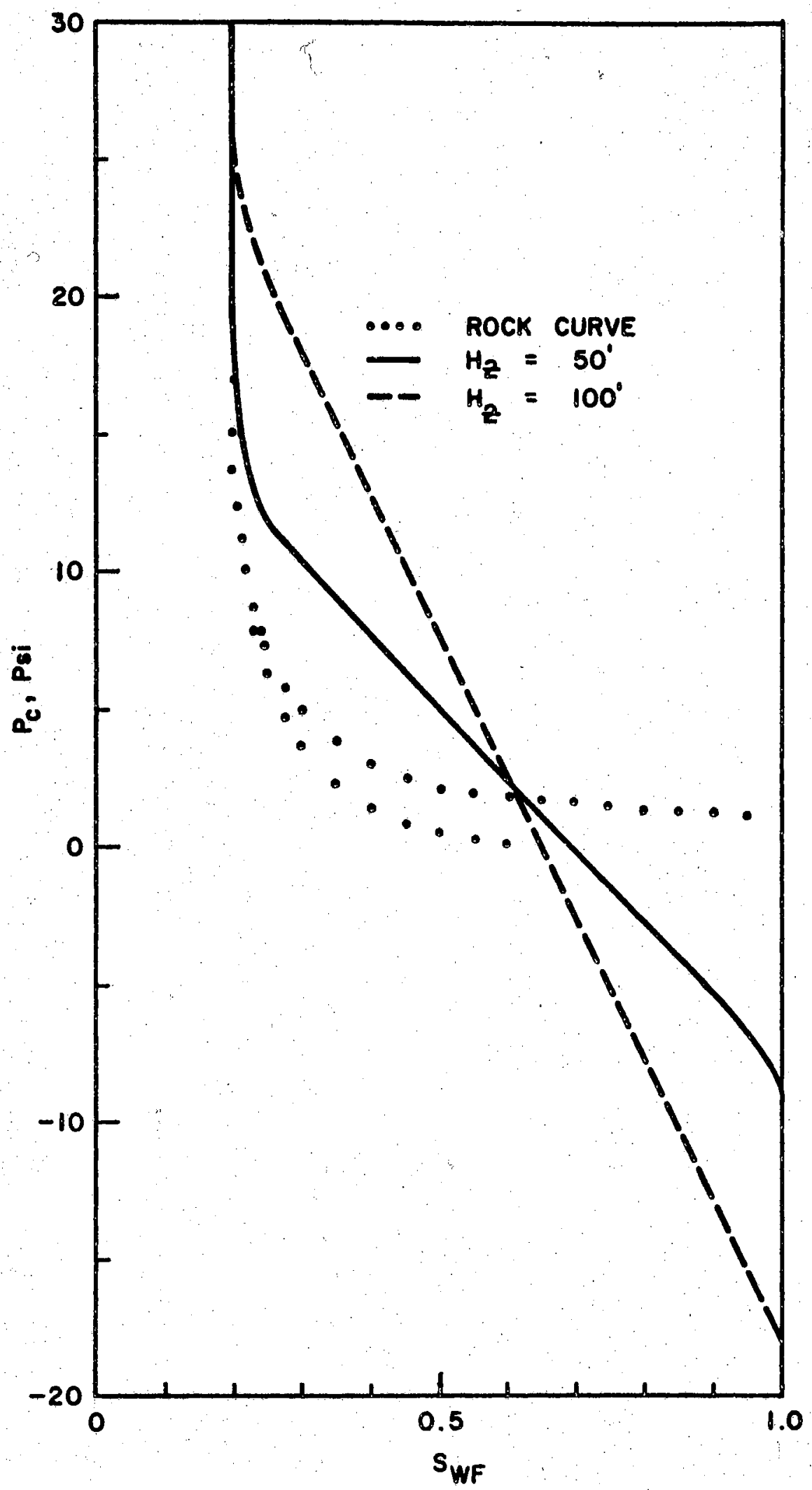

Figure 21. (Continued) 

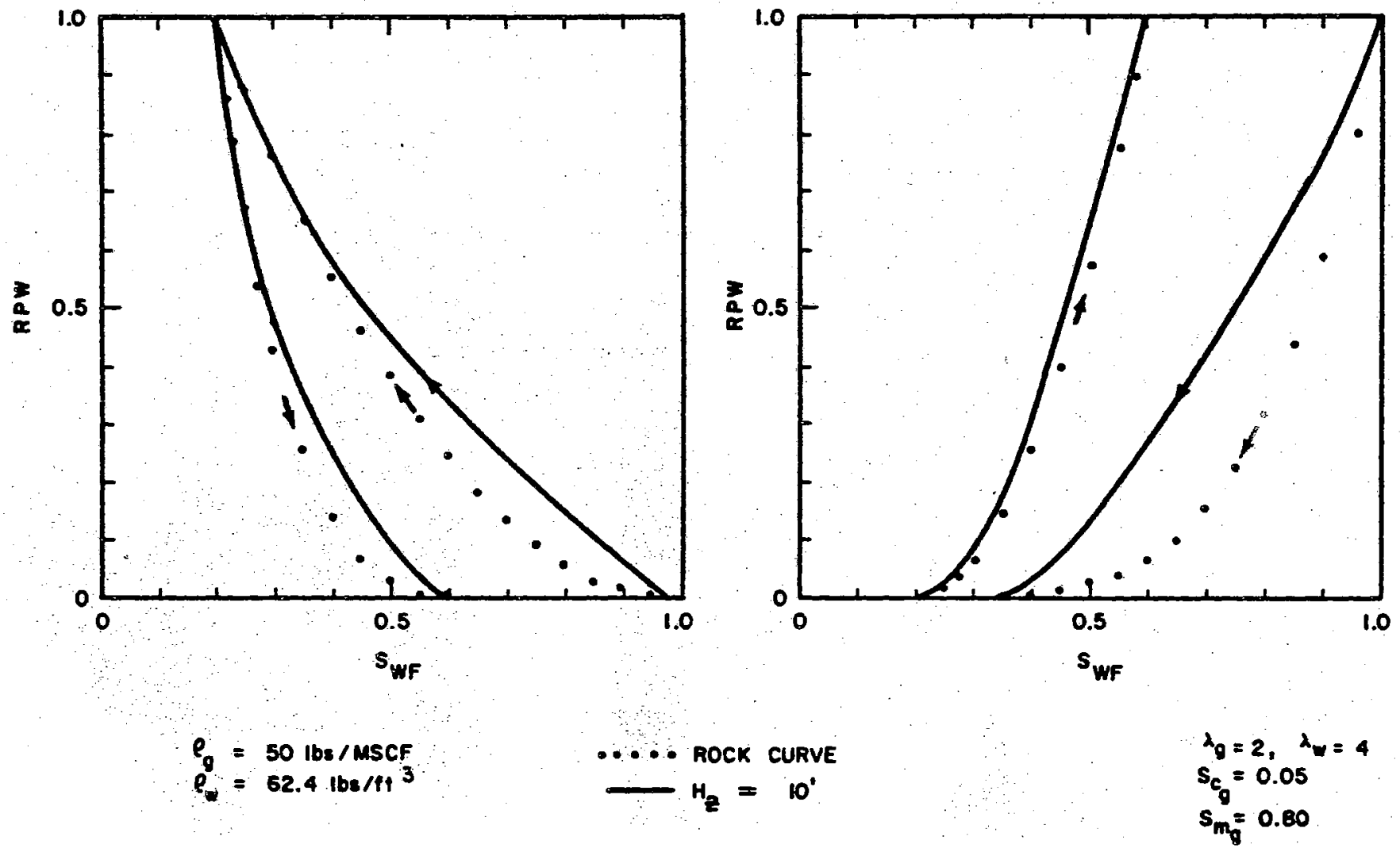

Figure 22. Sensitivity of Equilibrium Relative Permeability to Depth of Partial Integration in Dip-Normal Direction, $\mathrm{H}_{z}$ 


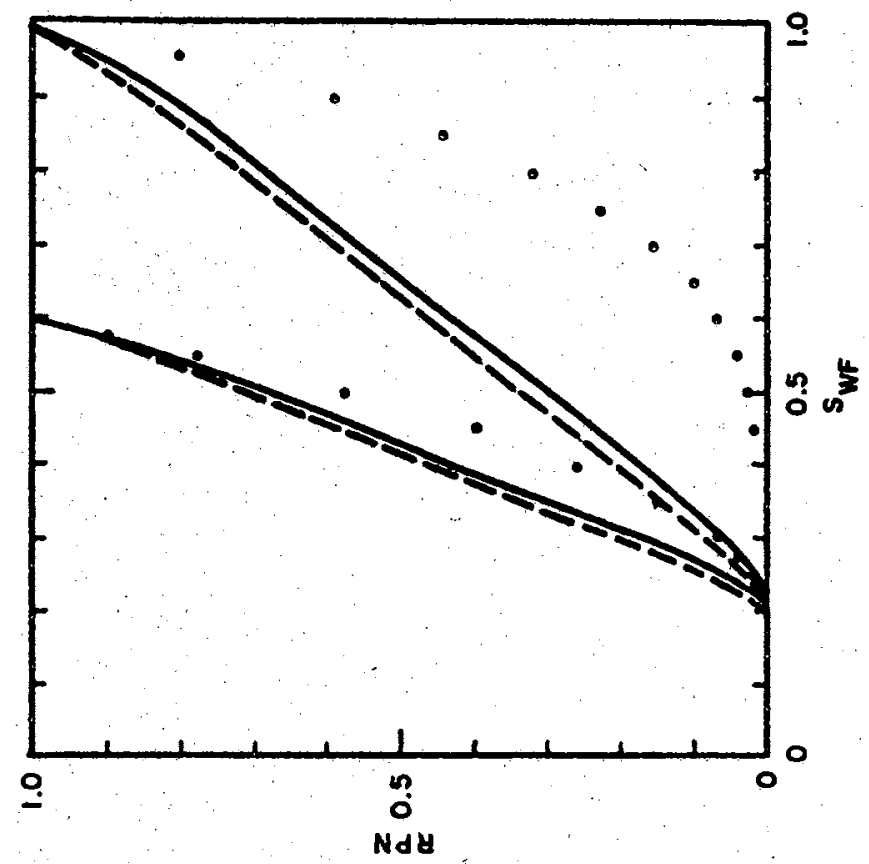

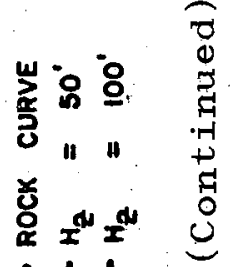

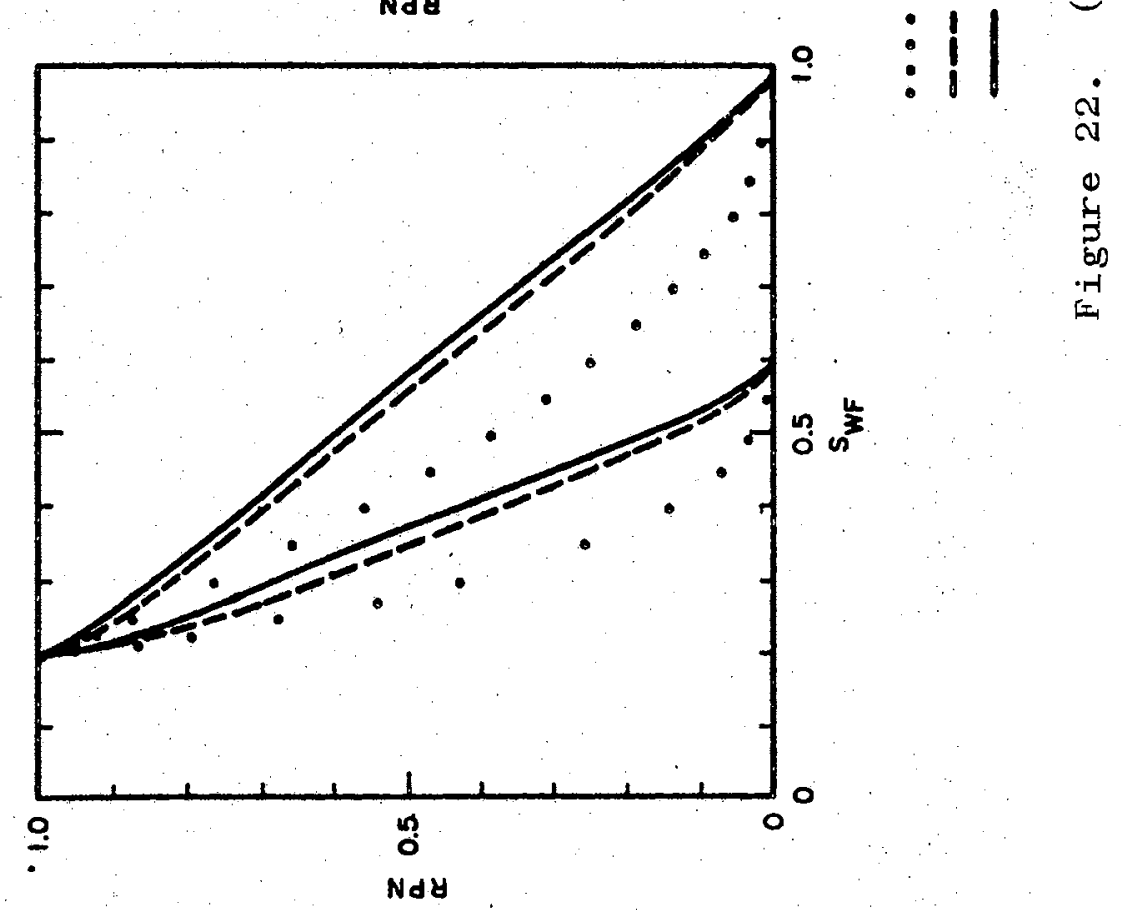


residual saturations can describe the equilibrium imbibition curves. The primary requirement is that dip-normal equilibrium (or near equilibrium) obtains.

If prototype reservoir conditions require the use of the three-dimensional simulator, the VE concept becomes valuable in reducing the grid spacing in dip-normal direction. For example, in studying layered, thick systems, one or more layers may be thicker than 10 feet, with flow conditions favoring dip-normal equilibrium. The saturation and pressure distributions would be in error if rock curves are used without adjustment. Simulation technologist would then face two alternatives; one is to set up sub-layers of 10 feet or less in thickness and use rock curves, the other is to adjust rock curves for dip-normal equilibrium. This adjustment, however, must be made with caution; because, the flow conditions permitting the partial integration in the dip-normal direction require that the layer (or reservoir as the case maybe) thickness be small relative to maximum distance across the reservoir. The main contribution of Run D1 was the demonstration of the fact that clever and realistic specification of the internal boundary conditions, hence the RP's of the blocks containing wells, are of first order importance in production simulation studies and accurate description of trapped gas volumes. 
Effect of Grid Size on Saturation and

Pressure Distributions

Simulation Run E1

The simulation Run E1, similar to Run B1 in every aspect except the size of the grid area was made to investigate the effects of additional grid blocks between well

blocks and grid system boundary on saturation and pressure distributions. Five additional blocks, $1000 \times 1000 \times 10$ feet in size, were placed beyond each boundary block of the first original grid system, while keeping the dip angle the same, $2^{0} 18^{*}$. This expansion amounted to moving the grid boundary 5000 feet into the infinite aquifer in $\mathrm{X}$ and $\mathrm{Y}$ directions, and 287 feet in $Z$ direction (see Figure 23).

The increase in the effective "radius" of the grid system from 5500 to 10,500 feet and the lowering of the structural position of this boundary another 287 feet below the top of the structure produced block dynamic pressure variations of the same order of magnitude as those attributable to $R P$ variations due to $\lambda$ and $S_{n}$; however, the saturation distribution was only slightly affected as shown below:

Cross section $I=5$

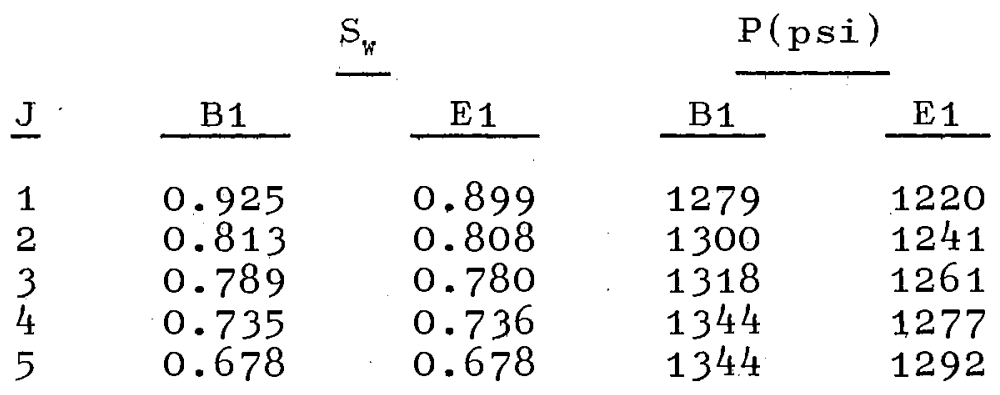




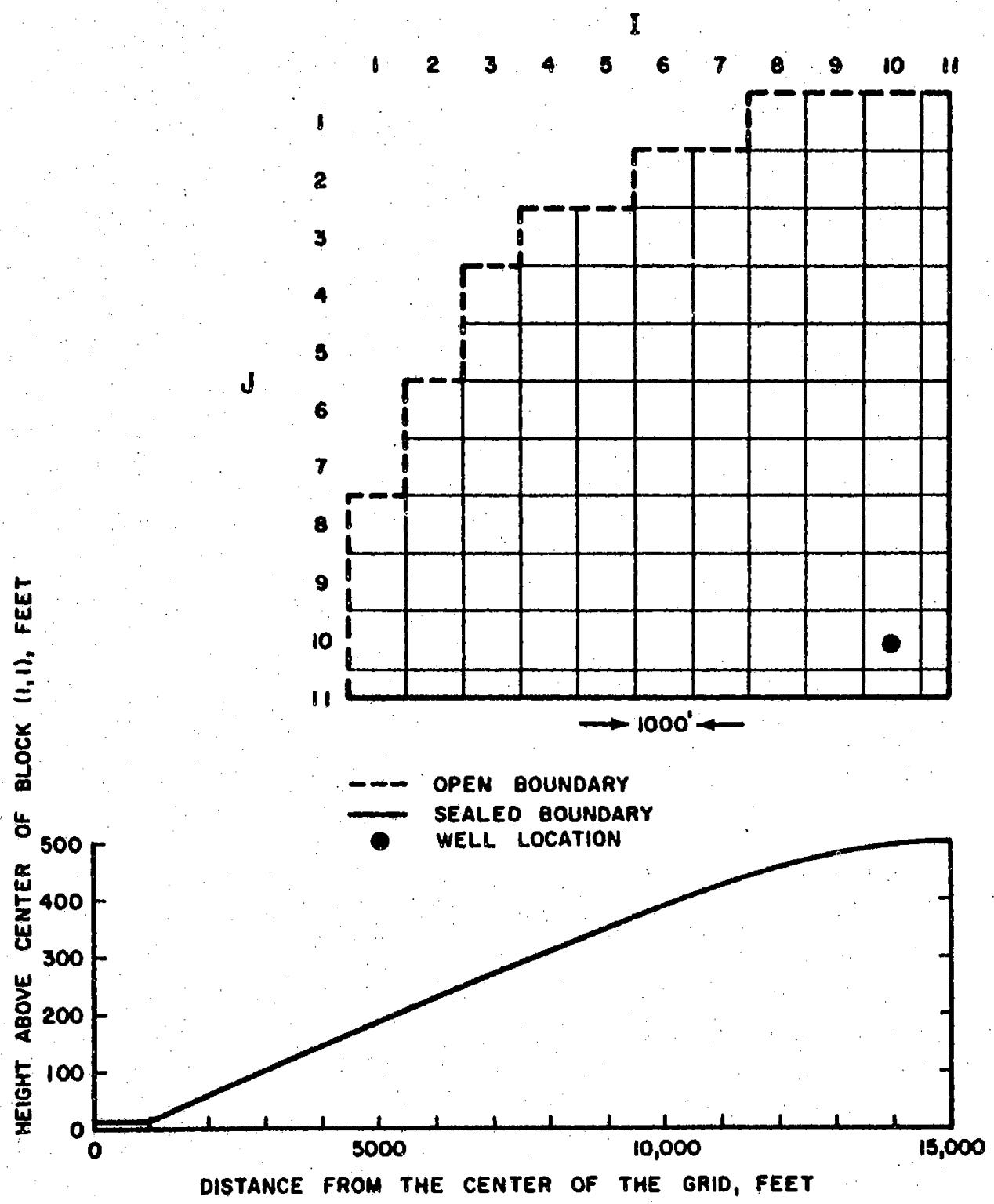

Figure 23. Two-Dimensional Grid Network Used in Simulation Run E1 
Simulation Run F1, F2, F3, F4, F5

These runs were made to investigate the influence of the grid block size on the saturation-pressure distribution sensitivity to RP-PC data. Radial grid-1 well option was used to model initial gas injection into the thin virgin aquifer described earlier for Runs B1 and E1. The grid system for Run $F 1$ was the finest, consisting of 117 rings (50 rings with 20 feet in incremental radius covering a 500-ft. radius about the well). Runs F2, F4, and F5 employed a total of 44 blocks (with twenty $30 \mathrm{ft}$. incremental radius rings covering a radial area of $500 \mathrm{ft}$. in radius). Run F3 (see Figure 24) used only 24 blocks (spanning the comparison area, $500 \mathrm{ft}$. , with only 11 blocks). Table VI illustrates saturation-pressure responses of the various runs to the injection of gas at a rate of 500 MSCF/D. Figure 25 shows the saturation distribution about the injection well at various levels of Run F3 simulation time.

Runs F1-F3 demonstrated that grid block size can affect the saturation distribution considerably without disturbing the pressure distribution (opposite of Run E1 findings). Runs F4 and F5 supported the results of the group B runs by displaying the sensitivity of the phase saturation distribution to $S_{e n}$ and to the curvature of the RP-S, PC-S.

In studies of initial gas injection into a thin aquifer with large rectangular-block grid systems, it is highly advisable to first use detailed grid (preferably radial) 


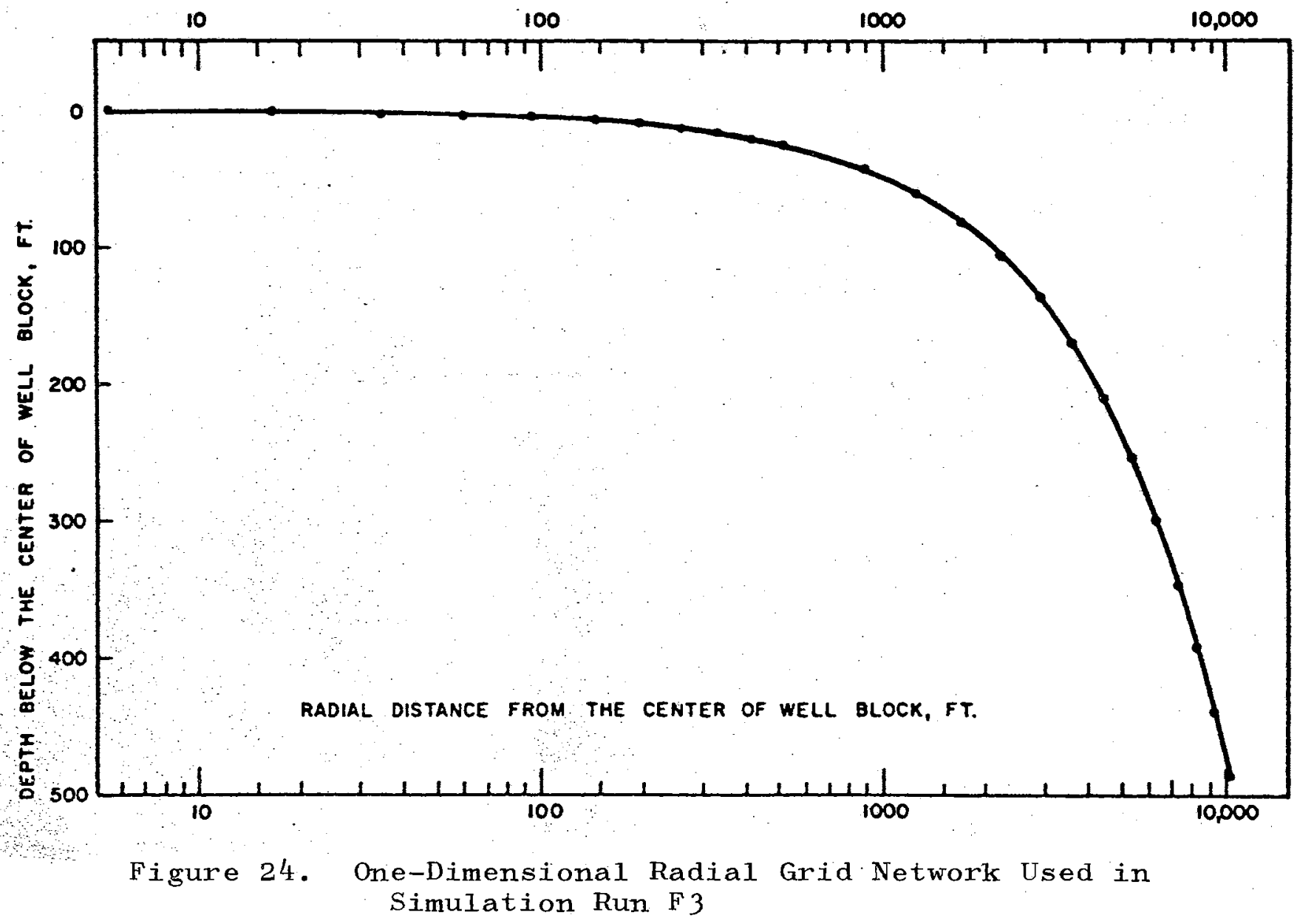


TABLE VI

SATURATION-PRESSURE DATA FROM RUNS F1-F5 AT

SIMULATION TIME LEVEL OF 50 DAYS

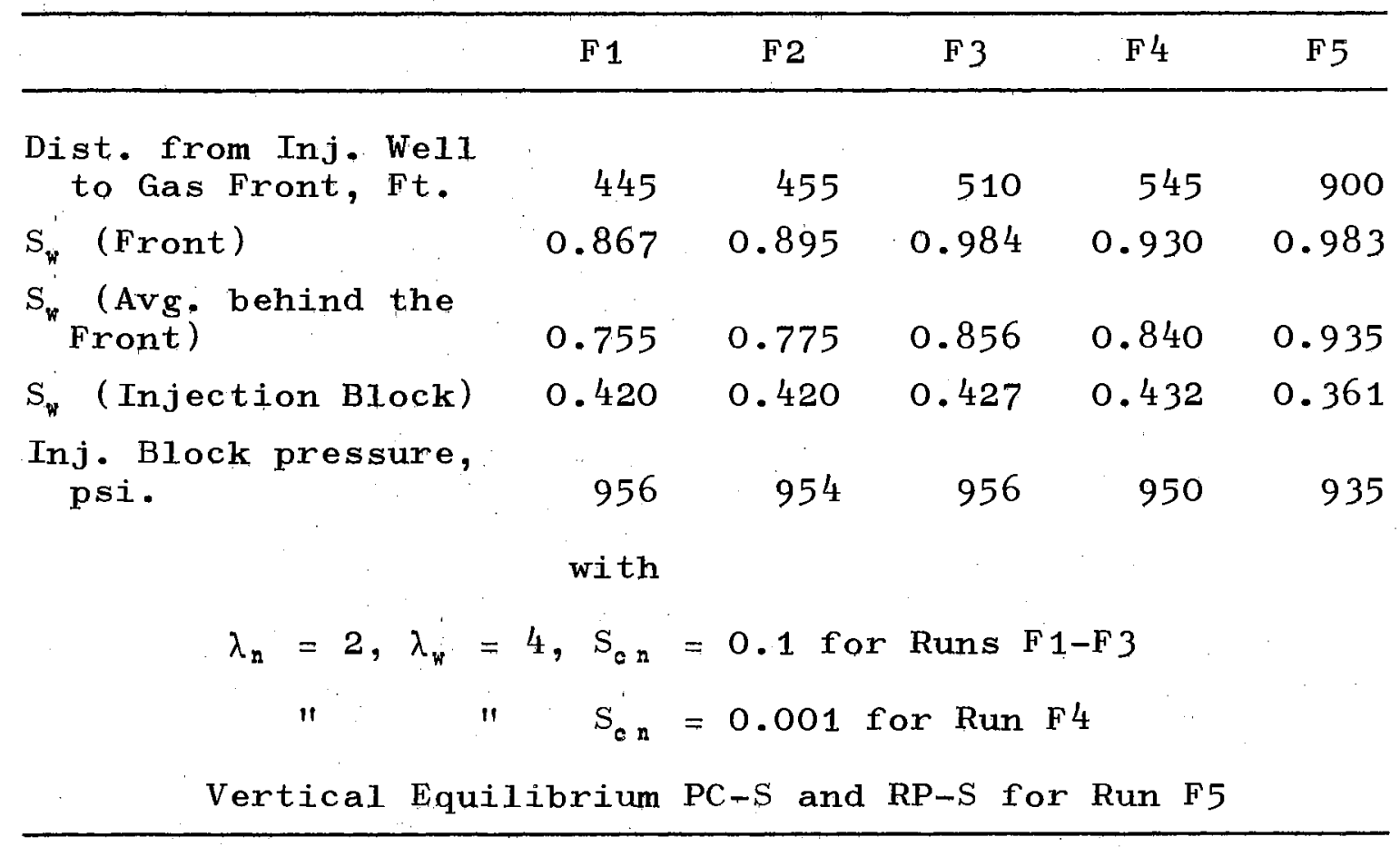



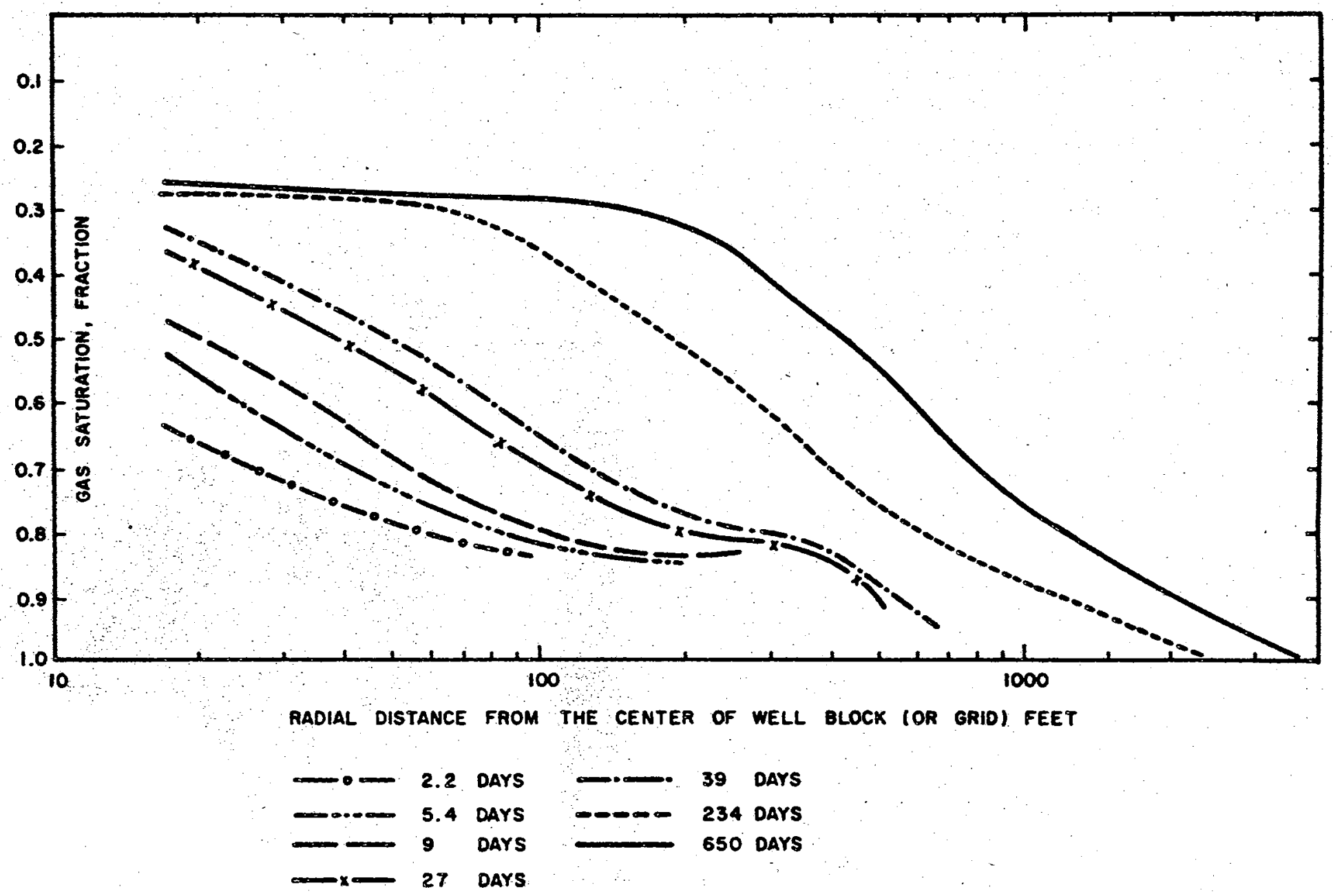

Figure 25. Saturation Distribution About the Injection Well at Various Levels of Run F3 Simulation Time 
models for typical wells in order to establish model critical saturations of regions covered by the well blocks and adjoining blocks. 


\section{APPENDIX F}

SYMBOL LIST FOR THE TEXT

A

$A_{x}, A_{y}$

B

B

$\mathrm{B}^{\prime}$

$C_{t}$

$\mathrm{D}(\mathrm{r})$

E

F

g

$g_{0}$

GIP

$\mathrm{H}_{\mathbf{k}}$

h
Area, $f t^{2}$.

Transmissibility, $\mathrm{L}^{3} / \mathrm{L}-\mathrm{T}$.

Characteristic constant of pore geometry, $1 / \mathrm{I}^{3}$

Formation volume factor, RBBL/STB or $\mathrm{RBBL} / \mathrm{MSCF}$.

$\mathrm{dB} / \mathrm{dP}$.

Combined compressibility of formation and water, psi $^{-1}$.

Distribution function for the radii of a bundle of capillary tubes.

Imbibition interference coefficient,

dimensionless.

Drainage interference coefficient,

dimensionless.

Acceleration of gravity, ft/sq-second.

Gravitational conversion constant,

$32.21 b_{m}-f t / 1 b_{q}-s q-$ second.

Gas in place, MSCF at standard conditions,

Iteration parameter for the $k^{\text {th }}$ iteration.

Reservoir thickness, ft. 
ID

IU

IM

IND

IWD

INI

IWI

$\mathrm{J}(\mathrm{S})$

$\underline{\mathrm{K}}$

k

k

$k_{\mathrm{g}}$

$k_{\text {s }}$

$\mathrm{L}_{\mathrm{x}}, \mathrm{L}_{\mathrm{y}}, \mathrm{L}_{\mathrm{z}}$

M

$\mathrm{M}_{\mathrm{o}}$

$N_{x}, N_{y}, N_{z}$

NWF, nwf

n

$\mathrm{P}, \mathrm{p}$

Pc, $p_{\text {o }}$

$P_{\odot x, z}^{*}$

Displacement process indicator, dimensionless. Universal indicator, dimensionless. Mobility indicator, dimensionless. Drainage RPN selector, dimensionless. Drainage RPW selector, dimensionless. Imbibition RPN selector, dimensionless. Imbibition RPW selector, dimensionless. Leverett's capillary pressure function, dimensionless.

Base permeability matrix.

Absolute (base) permeability, millidarcies. Iteration counter. Relative permeability, dimensionless. Pseudo-relative permeability, dimensionless. Spatial dimensions of the connected region, ft.

Mobility, $\left(\mathrm{k}_{\mathrm{r}} / \mu, \mathrm{cp}^{-1}\right)$

Capillary pressure multiplier, dimensionless. Number of grid blocks in $\mathrm{x}, \mathrm{y}$, and $\mathrm{z}$ directions.

Identifier for non-wetting phase.

Time step counter.

Pressure, psi.

Capillary pressure, psi.

Pseudo-capillary pressure at any areal point $(x, y)$ on the reference plane of the reservoir, psi. 


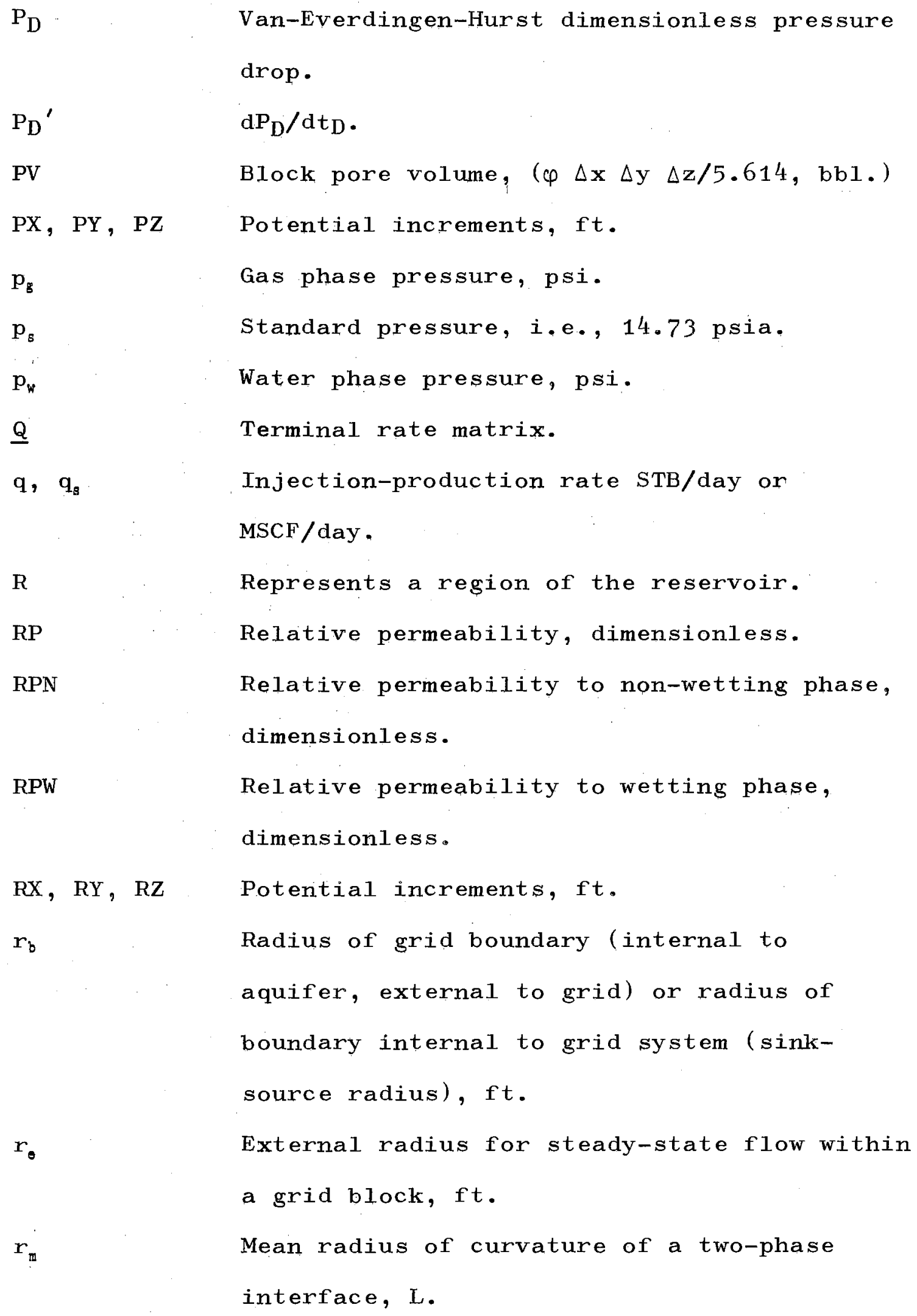




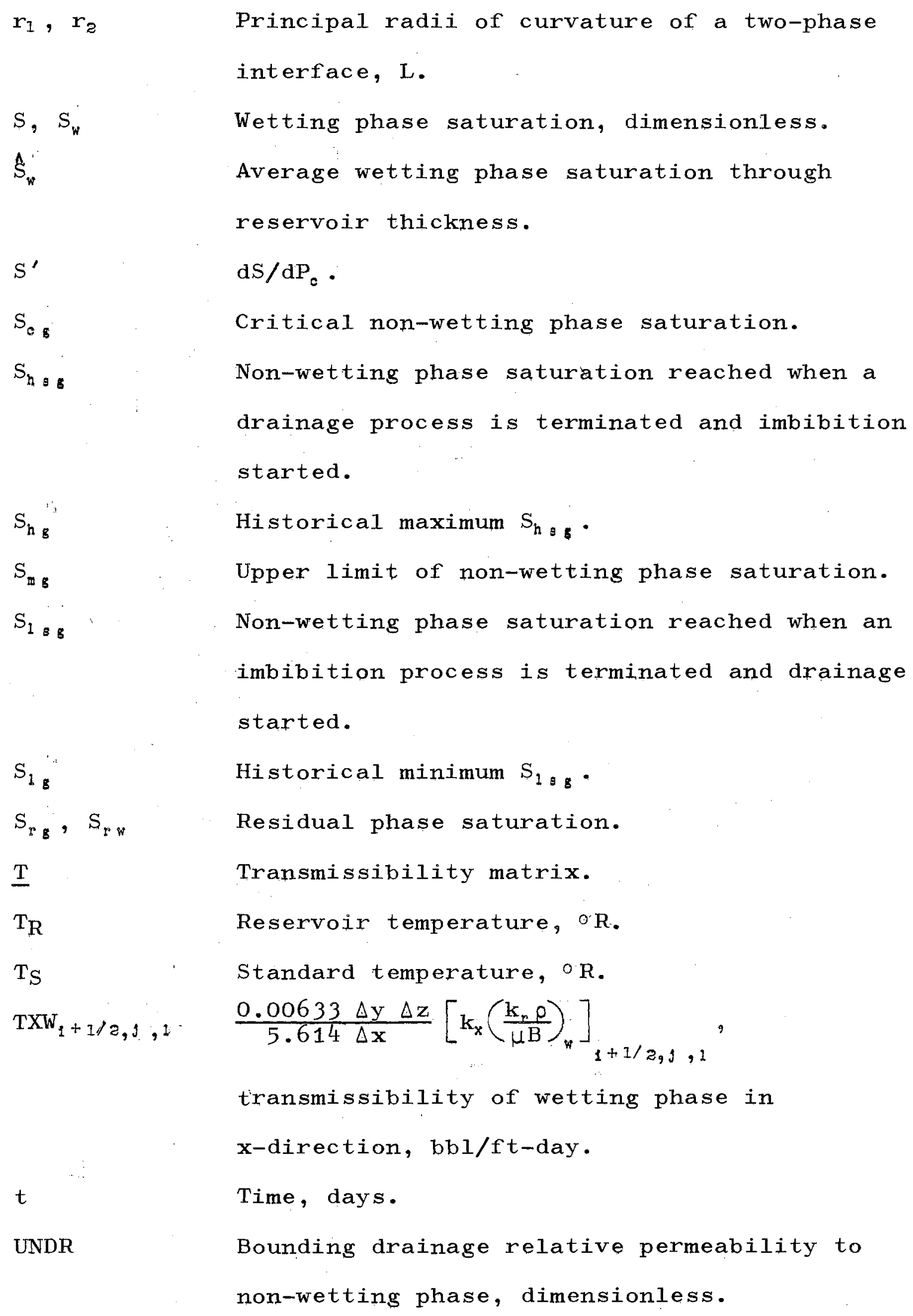


UNIM

UWDR

UWTM

$\bar{v}, \bar{u}$

$W_{e}$

WF, wf

WIP

$\Delta \mathbf{x}, \Delta \mathbf{y}, \Delta \mathbf{z}$

$Z_{g}, Z_{w}$

$\Delta$

E*

$\epsilon$

$\eta$

$\theta, \psi$

$\lambda$

$\mu$

$\nu$

$\pi$

$\rho$ or $\rho_{8}$
Bounding imbibition relative permeability to non-wetting phase.

Bounding drainage relative permeability to wetting phase.

Bounding imbibition relative permeability to wetting phase.

Darcy velocity, volume/sq. ft-day.

Cumulative Carter-Tracy type water influx, $\mathrm{bbl}$.

Identifier for wetting phase.

Water in place, bbl.

Grid block dimensions, ft.

Phase linkage coefficients for relative

permeability, dimensionless.

Difference operator.

The difference between true and numerical

solutions, potential units.

Tolerance, dimensionless.

$\cong \mathrm{n}+1$

Reservoir dip angle.

Drainage interference exponent for relative permeability, dimensionless.

Viscosity, centipoise.

Intermediate drainage interference exponent

for relative permeability, dimensionless. 3.1416

Specific weight, psi/ft, or density $\mathrm{Lb}_{\mathrm{m}} / \mathrm{ft} \mathrm{t}^{3}$. 
$\hat{p}$

$\sigma$

$T$

$\varphi$

$\varphi^{*}$

$\Phi$

$w$

Special symbols for the formulation of the differential and difference equations and numerical solution are defined in the text.

\section{SUBSCRIPTS}

f

$r, g$

w
Average specific weight over a time interval, psi/ft.

Interfacial tension, dynes/cm.

Intermediate imbibition interference

exponent for relative permeability,

dimensionless.

Porosity, fraction.

Transformed porosity, $\varphi\left(1-S_{F_{W}}\right)$, fraction.

Flow potential, ft.

Imbibition interference exponent,

dimensionless.

Denotes a vector.

Denotes a matrix. 


\author{
VITA \\ Attila Ismail Evrenos \\ Candidate for the Degree of \\ Doctor of Philosophy
}

Thesis: THE NUMERICAL SIMULATION OF HYSTERETIC FLOW IN
POROUS MEDIA

Major Field: Mechanical Engineering

Biographical:

Personal Data: Born in Istanbul, Turkey, February 19, 1937, the son of Em. Alb. Husnu H. and

Mihriban H. Evrenos.

Education: Graduated from Galatasaray Lyceum, Istanbul, Turkey, in 1956; attended University of Istanbul School of Economics in 1956; attended Santa Ana College, California, in 1957 and 1958; received Bachelor of Science degree from the University of Missouri at Rolla in 1961 with a major in Petroleum Engineering; attended Creighton University, Omaha, Nebraska, and University of Nebraska at Omaha from 1962 to 1965, on a part-time basis, with a major in Business Administration; received Master of Science degree from Oklahoma State University in 1967, as a Pan

American Oil Company Grant Fellow, with a major in Mechanical Engineering - Energy Resources Option; completed requirements for the Doctor of Philosophy degree at Oklahoma State University in May, 1970, as a Pan American Oil Company Grant Fellow.

Professional Experience: Employed by Northern Natural

Gas Company, Gas Supply Division, Omaha, Nebraska, as an engineer (trainee, junior, associate, senior levels) from June, 1961 to September, 1966; employed by Oklahoma State University as an Instructor in Petroleum Technology from September, 1966 to June, 1967, and from September, 1967 to June, 1968; self-employed as an engineering consultant to Northern Natural Gas Company, Omaha, 
Nebraska, and to Hydrocarbon Survey, Inc.,

Wichita, Kansas, from September, 1966 to June, 1968; now employed by Northern Natural Gas Company as an Applied Reservoir Research Engineer; also serving Hydrocarbon Survey, Inc., as a consultant.

Professional Organizations: Society of Petroleum Engineers of AIME, Operations Research Society of America, Phi Kappa Phi, Sigma Gamma Epsilon, Alpha Gamma Sigma. 